\title{
Abstracts From the 21st Annual HMO Research Network Conference, March 11-13, 2015, Long Beach, California
}

Follow this and additional works at: https://aah.org/jpcrr

Part of the Health and Medical Administration Commons, Medical Education Commons, and the Public Health Commons

\section{Recommended Citation}

Abstracts from the 21st annual HMO Research Network conference, March 11-13, 2015, Long Beach, California. J Patient Cent Res Rev. 2015;2(S1):S78-141. doi: 10.17294/2330-0698.1201

Published quarterly by Midwest-based health system Advocate Aurora Health and indexed in PubMed Central, the Journal of Patient-Centered Research and Reviews (JPCRR) is an open access, peer-reviewed medical journal focused on disseminating scholarly works devoted to improving patient-centered care practices, health outcomes, and the patient experience. 


\section{Abstracts From the $21^{\text {st }}$ Annual HMO Research Network Conference, March 11-13, 2015, Long Beach, California}

\section{CANCER}

B2-2:

\section{Diffusion of New Technologies for Patients With Prostate Cancer}

Ramzi G. Salloum, ${ }^{1}$ Matthew N. Nielsen, ${ }^{2}$ Mark C. Hornbrook, ${ }^{3}$ Maureen O'Keefe Rosetti, ${ }^{3}$ Paul A. Fishman, ${ }^{4}$ Jennifer Elston Lafata, ${ }^{5}$ Debra P. Ritzwoller ${ }^{6}$

${ }^{1}$ University of South Carolina; '2University of North Carolina at Chapel Hill; ${ }^{3}$ Kaiser Permanente Northwest; ${ }^{4}$ Group Health Cooperative; ${ }^{5}$ Virginia Commonwealth University; ${ }^{6}$ Kaiser Permanente Colorado

Background/Aims: Prostate cancer is the most common and costly cancer among U.S. men and offers a portfolio of case studies and clinical problems. Among these, the documented increases in use of robotic radical prostatectomy (RRP) and intensity-modulated radiotherapy (IMRT) are illustrative of rapid diffusion of costly, unproven technological innovations. Evidence of these case studies has been limited to the traditional Medicare fee-for-service (FFS) aged population and may provide a potentially biased view of the universe of cancer care in the United States. To address this issue, we examine treatment patterns among prostate cancer patients enrolled in two integrated health care delivery systems.

Methods: The sample included a retrospective cohort of patients diagnosed with nonmetastatic prostate cancer between 2000 and 2008 at the Northwest and Colorado regions of Kaiser Permanente. Choice of radiation therapy was defined as the receipt of external beam radiotherapy (threedimensional [3D] conformal radiation therapy vs. IMRT) as primary treatment within one year of diagnosis. Choice of prostatectomy was defined as the receipt of surgery as primary treatment (open radical prostatectomy vs. minimallyinvasive/robotic radical prostatectomy). A pooled time-series cross-section design was used to analyze the medical care resource use for treating prostate cancer.

Results: Both RRP and IMRT were introduced in the two health systems in 2003, and their use has been steadily increasing since then. Use of traditional treatments has either remained stable: open radical prostatectomy; or decreased: 3D conformal radiation therapy.

Discussion: We hypothesize that diffusion rates of RRP and IMRT have been slower among HMO enrollees compared with FFS patients. This study improves our understanding of patterns of prostate cancer care in the United States by using a large sample of managed care enrollees across the age spectrum, including men younger than 65 relative to men older than 65 years.

Keywords: prostate, technology
B2-4:

An Economic Evaluation of Colorectal Cancer Screening in Primary Care Practice

Richard T. Meenan, ${ }^{1}$ Jessica Chubak, ${ }^{2}$ Sally W. Vernon, ${ }^{3}$ Sharon Fuller, ${ }^{2}$ Ching-Yun Wang, ${ }^{4}$ Beverly B. Green ${ }^{2}$

${ }^{1}$ Kaiser Permanente Northwest; ${ }^{2}$ Group Health Cooperative; ${ }^{3}$ University of Texas; ${ }^{4}$ Fred Hutchinson Cancer Research Center

Background/Aims: This study evaluated the costeffectiveness of interventions using electronic health records (EHR), automated mailings, and stepped increases in support to improve two-year colorectal cancer screening adherence.

Methods: Analyses are based on a parallel-design, randomized trial in which three stepped interventions (EHR-linked mailings ["automated"], automated plus telephone assistance ["assisted"], or automated and assisted plus nurse navigation to testing completion or refusal ["navigated"]) were compared to usual care. Data were collected over August 2008-November 2011 with analysis in 2012-2013. Implementation resources were micro-costed; research and registry development costs were excluded. Incremental cost-effectiveness ratios (ICERs) were based on number of participants current for screening per guidelines over two years. Robustness of results was examined through bootstrapping.

Results: Intervention delivery cost per participant current for screening ranged from \$21 (automated) to \$27 (navigated). When induced testing costs (e.g. screening colonoscopy) were included, automated (ICER: -\$159) and assisted (ICER: -\$36) were cost-saving relative to usual care. Savings arose from increased fecal occult blood testing, substituting for more expensive colonoscopies in usual care. Results were broadly consistent across demographic subgroups. More intensive interventions were consistently likely to be cost-effective relative to less intensive interventions with willingness to pay values of $\$ 600-\$ 1,200$ for a $1 \%$ increase in the rate of screening adherence yielding probability of cost-effectiveness of at least $80 \%$.

Discussion: The cost-effectiveness was demonstrated of a stepped approach to colorectal cancer screening promotion, especially one using automated data systems linked to electronic health records.

Keywords: colorectal cancer screening, cost-effectiveness

B2-5:

Randomized Trial of In-Home Cervical Cancer Screening in Underscreened Women

Rachel L. Winer, ${ }^{1}$ Jasmin A. Tiro, ${ }^{2}$ Diana L. Miglioretti, ${ }^{1}$ Chris Thayer, ${ }^{1}$ Jane A. Dimer, ${ }^{1}$ John Lin, ${ }^{3}$ Hongyuan Gao, ${ }^{1}$ Diana S. Buist ${ }^{1}$ 
${ }^{1}$ Group Health Cooperative; ${ }^{2}$ University of Texas Southwestern; ${ }^{3}$ University of Washington

Background/Aims: Over half of cervical cancers in the United States are diagnosed in unscreened or underscreened women. Innovative strategies that move primary screening out of the clinic could be highly effective in improving screening compliance while maintaining high-quality care. Internationally, there is growing interest in a primary screening strategy of self-collected, in-home high-risk human papillomavirus (hrHPV) screening followed by triage of hrHPV-positive results to clinician-collected cytology or colposcopy. The need for in-clinic screening could be eliminated for a majority of women in organized screening programs if hrHPV screening can be seamlessly integrated with in-clinic follow-up.

Methods: In March 2014, we launched a large, pragmatic randomized controlled trial within Group Health to compare effectiveness of two programmatic approaches for increasing cervical cancer screening among 17,000 women aged 30-64 years who are overdue $(\geq 3.4$ years since last Pap test or never had a Pap test). The control arm is usual care at Group Health, consisting of an organized program of patient invitation and reminders and electronic medical record (EMR)-based alerts to providers about patients who are not up-to-date with recommended screening. The intervention arm includes usual care plus a mailed in-home hrHPV screening kit. Women mail their screening kits directly to our centralized laboratory, and hrHPV test results are documented in our EMR to notify women's provider teams of appropriate follow-up care. Primary outcomes are early detection and treatment of cervical neoplasia. Secondary outcomes are cervical cancer screening uptake and screening predictors, and patient experiences and attitudes towards in-home hrHPV testing and follow-up of hrHPV-positive results. We are surveying 100 responders and 100 nonresponders to the in-home hrHPV screening invitation, and interviewing 50 women with hrHPV-positive results (including those with timely versus late completion of clinical follow-up).

Results: As of September 2014, 6,194 eligible women were identified and randomized. We will highlight the study design and lessons learned from implementing a pragmatic trial.

Discussion: This pragmatic trial will generate evidence-based data on the impact of an in-home hrHPV screening program in a real-world clinical setting. Findings will interest organized programs considering implementing in-home screening and low-resource countries caring for populations with limited access to primary care.

Keywords: cervical cancer, cancer screening

B3-1:

The CRN Scholars Program: Career Development Within CRN

Matthew P. Banegas, ${ }^{1}$ Diana S. Buist ${ }^{2}$ Mara M. Epstein, ${ }^{3}$ Terry S. Field, ${ }^{3}$ Grace Clarke Hillyer, ${ }^{4}$ Jason P. Lott, ${ }^{5}$ Pamala A. Pawloski ${ }^{6}$
${ }^{1}$ National Cancer Institute; ${ }^{2}$ Group Health Cooperative; ${ }^{3}$ University of Massachusetts Medical School; ${ }^{4}$ Columbia University; ${ }^{5}$ Yale School of Medicine; ${ }^{6}$ HealthPartners

Background/Aims: The Cancer Research Network (CRN) Scholars Program was launched during the third cycle of the collaborative grant to increase research capacity within the CRN. Specific goals of the program include: (1) becoming a principal investigator on a successful investigator-initiated grant within the CRN, and (2) primary author on peerreviewed, published manuscripts reporting original research from the CRN. In addition to developing research expertise utilizing CRN resources, the CRN Scholars Program fosters development of skills difficult to acquire in other training environments, including the initiation of collaborative research proposals involving stakeholders in integrated health care delivery systems, use and analysis of complex clinical, claims and pharmaceutical data, and strategic grant proposal development tailored to the unique resources and opportunities afforded by the CRN.

Methods: The 26-month program facilitates individualized mentorship, creation of research advisory teams for each scholar comprised of investigators from their home institution and collaborating CRN sites. Monthly one-on-one mentoring meetings and quarterly mentoring team meetings enable scholars to gain familiarity with the CRN landscape to develop novel, investigator-initiated projects with CRN collaborators. Additionally, scholars attend semiannual in-person meetings and conduct bimonthly group conference calls examining academic career development, writing and review of research proposals, analytic design, discussing available funding opportunities, execution of multisite research studies, and media training.

Results: The first two Scholars Program cohorts included $\mathrm{CRN}$-affiliated junior investigators, while the current iteration of the Scholars Program (CRN4) has shifted focus to include external junior investigator researchers pursuing training in the conduct of multisite, multidisciplinary population-based studies. Members of the CRN4 cohort have successfully received career development awards and independent grants to support their CRN work, published several first-authored manuscripts and received awards honoring their research.

Discussion: The CRN Scholars Program is working to expand the scope and scale of investigators conducting cancer research within integrated health care delivery systems, a goal of significant importance given nationwide shifts in policy and practice in the organization of medical care and emphasis on population health. Accordingly, the CRN Scholars Program is well positioned to produce future leaders in cancer research and, ultimately, improve the lives of patients.

Keywords: scholarship, training

C2-5:

Using Natural Language Processing to Identify Health Plan Beneficiaries With Pulmonary Nodules

Farhood Farjah, ${ }^{1}$ Scott Halgrim, ${ }^{2}$ Steven B. Zeliadt, ${ }^{3}$ Michael K. Gould, ${ }^{4}$ David S. Carrell, ${ }^{2}$ Elizabeth T. Loggers, ${ }^{5}$ David R. Flum, ${ }^{1}$ Diana S. Buist ${ }^{2}$ 
${ }^{1}$ University of Washington; ${ }^{2}$ Group Health Cooperative; ${ }^{3}$ Veterans Administration Puget Sound Health Care System; ${ }^{4}$ Kaiser Permanente Southern California; ${ }^{5}$ Seattle Cancer Care Alliance

Background/Aims: The development of a portable, automated method for identifying individuals with lung nodules will facilitate the efficient conduct of populationbased studies of nodule care and associated outcomes. We evaluated the performance of a previously developed natural language processing (NLP) algorithm for identifying health plan beneficiaries with pulmonary nodules.

Methods: A cross-sectional study was performed of 500 randomly selected adult, in-network health plan beneficiaries with continuous enrollment at Group Health Cooperative who underwent a computed tomography (CT) of the chest in 2012, had no history of lung cancer and had not undergone a CT between 2009 and 2011. An NLP algorithm originally developed at Kaiser Permanente Southern California assessed electronic radiology reports using keywords and qualifiers relating to pulmonary nodules ranging in size from 5 to $30 \mathrm{~mm}$ among individuals who had undergone CT and had an International Classification of Diseases (ICD-9-CM) diagnostic code for a lung nodule. This algorithm was applied to our patient population and modified to identify pulmonary nodules regardless of size. A trained chart abstractor reviewed radiology reports to determine whether the radiologist reported a lung nodule. An experienced, board-certified thoracic surgeon adjudicated radiology reports with unclear documentation of a nodule.

Results: The true prevalence of pulmonary nodules among individuals undergoing CT in 2012 - median age 65 years, $43 \%$ men, $84 \%$ white, $51 \%$ smokers - was $34 \%$. Median nodule size was $6 \mathrm{~mm}$ (range 2-87 mm). NLP identified 218 $(44 \%)$ individuals with a nodule. The accuracy of NLP was as follows: sensitivity $91 \%$, specificity $81 \%$, positive predictive value $72 \%$ and negative predictive value $95 \%$.

Discussion: An automated method of using NLP and electronic radiology text reports - originally developed at one Cancer Research Network (CRN) site - reasonably identifies health plan members with pulmonary nodules at another CRN site. This finding supports the notion that automated methods are portable across integrated health systems and institutions using electronic medical records. Ongoing work seeks to determine whether modifications to the NLP algorithm can improve performance. Given its current performance characterized by a high negative predictive value, NLP could be used to decrease the burden of chart abstraction in population-based studies of nodule care.

Keywords: pulmonary nodule, natural language processing

D3-5:

Long-Term Medical Care Costs of Breast, Prostate, Lung and Colorectal Cancer for HMO Members

Matthew P. Banegas, ${ }^{1}$ K. Robin Yabroff, ${ }^{1}$ Maureen O'Keefe Rosetti, ${ }^{2}$ Debra P. Ritzwoller, ${ }^{3}$ Paul A. Fishman, ${ }^{4}$ Ramzi G. Salloum, ${ }^{5}$ Jennifer Elston Lafata, ${ }^{6}$ Mark C. Hornbrook $^{2}$
${ }^{1}$ National Cancer Institute; ${ }^{2}$ Kaiser Permanente Northwest; ${ }^{3}$ Kaiser Permanente Colorado; ${ }^{4}$ Group Health Cooperative; ${ }^{5}$ University of South Carolina; ${ }^{6}$ Virginia Commonwealth University

Background/Aims: The rising economic burden of cancer in the United States has become a significant public policy issue. Current knowledge of cancer care costs is largely limited to Medicare fee-for-service patients age $\geq 65$ years, based on linked Surveillance, Epidemiology, and End Results (SEER) Medicare data. Accordingly, estimates that reflect care costs across the cancer experience, from diagnosis to end-of-life and among individuals $<65$ years of age, are needed. This study estimated total and incremental long-term costs of care for individuals age $\geq 18$ years diagnosed with breast, colorectal, lung and prostate cancer in integrated health care settings.

Methods: We used administrative and electronic medical record data linked with tumor registry information for individuals who were enrolled in one of four nonprofit integrated health care systems and diagnosed with primary breast, colorectal, lung or prostate cancer between 1/1/200012/31/2008 (cases). We also identified age-/sex-matched cancer-free individuals (controls). Participating health systems included Group Health Cooperative, Henry Ford Health System, Kaiser Permanente Northwest and Kaiser Permanent Colorado. Long-term costs (at 5,10 and 20 years) of care were based on the Phases-of-Care approach (Initial, Continuing and End-of-life phases) and measured using the Kaplan-Meier Sample Average estimator, incorporating cost data from the standardized relative resource cost algorithm and cancer site-, age- and stage-specific survival probabilities from SEER. Costs were calculated for both cases and controls to estimate total and incremental care costs, stratified by cancer site, age group ( $<65$ vs. $\geq 65$ years) and stage at diagnosis (advanced vs. not advanced).

Results: Total and incremental long-term costs of cancer care were significantly higher among individuals age $<65$ years at diagnosis compared to those $\geq 65$ years. There was considerable variability in total and incremental long-term costs by cancer site and stage at diagnosis.

Discussion: Estimates reported in this study improve our understanding of the magnitude of long-term economic resources needed to provide cancer care in the United States by describing cancer care costs among patients in integrated care settings and those age $<65$ years. Our results will be directly applicable to future analyses of cancer care costs, serving as a foundation for cost comparisons across different health care systems.

Keywords: cost of care, cancer

ECl-3:

Disparities in Colorectal Cancer Screening Rates Among Asian Subgroups in a Large Managed Care Organization

Nirupa R. Ghai, ${ }^{1}$ Douglas A. Corley, ${ }^{2}$ Chyke A. Doubeni, ${ }^{3}$ Christopher D. Jensen, ${ }^{2}$ Joanne E. Schottinger, ${ }^{1}$ Ann G. Zauber, ${ }^{4}$ Theodore R. Levin, ${ }^{2}$ Virginia P. Quinn ${ }^{1}$ 
${ }^{1}$ Kaiser Permanente Southern California; ${ }^{2}$ Kaiser Permanente Northern California; ${ }^{3}$ University of Pennsylvania; ${ }^{4}$ Memorial Sloan Kettering Cancer Center

Background/Aims: Colorectal cancer (CRC) is the second leading cause of cancer death in the United States. CRC screening allows for early detection of CRC or its precursors, and leads to a decrease in CRC incidence and mortality. National guidelines recommend screening with either an annual fecal immunochemical test (FIT), a colonoscopy every 10 years, or a flexible sigmoidoscopy every 5 years for individuals age 50-75 years. Nationally, the overall age-adjusted combined CRC screening rates increased from $52.3 \%$ to $65.4 \%$ between 2002 and 2010; however, screening rates among Asian Americans were 11\% lower compared with whites, but differences among Asian subgroups is unknown. Thus, we examined CRC screening rates among Asian subgroups in a large managed care setting.

Methods: We used electronic data to characterize CRC screening among Asian subgroups (Japanese, Korean, Filipino, Asian-Indian, Chinese, Vietnamese, Other Asian) compared with white non-Hispanics (WNHs). Using descriptive and multivariable models, we evaluated 408,242 screening-eligible members of Kaiser Permanente Southern California aged 50-89 years as of January 1, 2010.

Results: Compared with WNHs over the past 3 years, Japanese were least likely and Koreans were most likely to have had any CRC screening exam (odds ratio [OR] 0.89, 95\% confidence interval [CI] 0.84-0.95; and OR 1.84, 95\% CI 1.68-2.02, respectively). Of those screened, compared with WNHs, Japanese men were older (mean age 65.2 vs. 62.6). Screened Asian-Indians and Vietnamese had an equal distribution of men and women, while the other subgroups had more females. In the previous 3 years, Japanese were less likely to have had a FIT $(41 \%, 1,742$ of 4,301$)$, and Vietnamese were more likely $(56 \%, 2,248$ of 4,018$)$. In the previous 10 years, Koreans were more likely to have had a colonoscopy $(56 \%, 1,797$ of 3,189) and Filipinos were least likely $(42 \%, 9,135$ of 21,688$)$.

Discussion: CRC screening rates and choice of screening test differ among Asian subgroups in an insured population. Further research is needed to understand the reasons for the differences among Asian subgroups, particularly the Japanese population.

Keywords: colorectal cancer screening, health care disparities

PD2-1:

Trends in Estrogen Receptor Testing and Tamoxifen Initiation After Ductal Carcinoma in Situ During 1996-2011

Hazel B. Nichols, ${ }^{1}$ Erin J. Bowles, ${ }^{2}$ Diem-Thy Tran, ${ }^{2}$ Diana S. Buist ${ }^{2}$

\section{${ }^{1}$ University of North Carolina; ${ }^{2}$ Group Health Cooperative}

Background/Aims: Ductal carcinoma in situ (DCIS), a stage 0 breast cancer usually detected on mammogram, accounts for $24 \%$ of all breast cancer diagnoses. In 2000, tamoxifen was FDA-approved as adjuvant endocrine therapy for DCIS patients. We assessed tamoxifen initiation after DCIS diagnosis according to calendar year, tumor characteristics and concurrent treatment within Group Health Cooperative (GHC).

Methods: We identified female GHC enrollees aged 18-89 years with an incident DCIS diagnosis during 1996-2011. Eligibility criteria required 12 months continuous enrollment before and after DCIS diagnosis. Tamoxifen initiation was identified through pharmacy records. Tumor characteristics and treatment information were captured from the virtual data warehouse. Relative risks (RR) and 95\% confidence intervals (CI) for tamoxifen initiation were calculated with multivariable generalized linear models adjusted for age, calendar year, estrogen receptor (ER) status, radiation therapy and grade.

Results: We identified 727 women with a DCIS diagnosis, including $163(22 \%)$ who initiated endocrine therapy within 12 months. Fourteen women filled aromatase inhibitor prescriptions and were analyzed with tamoxifen users. ER testing increased from 4\% of DCIS cases in 2001 to $71 \%$ in 2011. Women diagnosed with DCIS during 1996-2000 were $43 \%$ less likely to use tamoxifen as those diagnosed in 2001-2005 (RR: 0.6, CI: 0.4-0.9). However, tamoxifen use did not vary significantly across calendar years after 2001 . Among ER-positive tumors, 58\% (18 of 31) used tamoxifen in 2001-2005 compared to 39\% (47 of 122) in 2006-2011. Among women with unknown ER status, 24\% (47 of 194) used tamoxifen in 2001-2005 compared to $15 \%$ (21 of 140) in 2006-2011. Younger women were more likely to use tamoxifen (RR: $1.7,95 \%$ CI: 1.1-2.6 for age 45-54 compared to $65-74$; P-trend=0.0002). Compared to breast conserving surgery (BCS) with radiation, women who had BCS without radiation (RR: 0.5 , CI: $0.2-0.9$ ) or mastectomy (RR: 0.6 , CI: $0.4-0.8)$ were less likely to use tamoxifen. Six percent $(n=47)$ of women had a bilateral mastectomy; of these, $<5$ used tamoxifen.

Discussion: Increasing ER testing since 2001 has not corresponded to parallel increases in tamoxifen use. Tamoxifen use after DCIS was more common among younger women and those who had BCS and radiation therapy.

Keywords: tamoxifen, DCIS

\section{PD2-3:}

Tamoxifen and Antidepressant Drug Interactions in a Large Cohort of Breast Cancer Survivors

Reina Haque, ${ }^{1}$ Jiaxiao Shi, ${ }^{1}$ Suzanne Fletcher, ${ }^{2}$ Joanne E. Schottinger, ${ }^{1}$ Syed A. Ahmed, ${ }^{1}$ T. Craig Cheetham, ${ }^{1}$ Joanie Chung, ${ }^{1}$ Chantal Avila, ${ }^{1}$ Laurel A. Habel, ${ }^{3}$ Marilyn L. Kwan ${ }^{3}$

\section{${ }^{1}$ Kaiser Permanente Southern California; ${ }^{2}$ Harvard Pilgrim Health Care; ${ }^{3}$ Kaiser Permanente Northern California}

Background/Aims: Considerable controversy exists on whether certain antidepressants reduce tamoxifen's effectiveness in lowering recurrence or subsequent breast 
cancer. Previous studies have been limited by small sample sizes, poor measurement of medication use, or inadequate statistical analysis. Our goal was to determine whether taking tamoxifen and antidepressants concomitantly is associated with an increased risk of subsequent breast cancer in survivors. Methods: We assembled a cohort of 16,887 women who were diagnosed with their first primary breast cancer (Stage 0-II) from 1996 to 2007 and treated with tamoxifen and followed through 12/31/2009 at Kaiser Permanente Southern and Northern California. We collected demographic, tumor, pharmacy and cancer treatment information. The outcome measure was risk of subsequent breast cancer. The main independent variables were fractions of overlap when both tamoxifen and antidepressant treatments were prescribed. Hazard ratios (HR) and 95\% confidence intervals (CI) were estimated using Cox regression models.

Results: Overall, 2,946 women (17\%) developed subsequent breast cancer during the 14-year study period. We observed a small nonsignificant increased risk of subsequent breast cancer in women who concurrently used paroxetine in the first full year of tamoxifen use. For every $25 \%$ increase in percent days overlap of tamoxifen and paroxetine use, the risk increased insignificantly by $6 \%$ (HR: $1.06,95 \%$ CI: $0.98-$ 1.14). However, the risk attenuated with longer tamoxifen duration.

Discussion: We observed a slight increased risk of subsequent breast cancer in women who concurrently used paroxetine in the first year of endocrine therapy. Nevertheless, taking tamoxifen for a longer duration mitigated such risks.

Keywords: breast cancer, medical treatment

\section{PD2-6: \\ Data Mining via the Electronic Medical Record to Identify Inherited Colorectal Cancer in an HMO Patient Population}

Monica Alvarado, ${ }^{1}$ Hilary Kershberg, ${ }^{1}$ George Tiller, ${ }^{1}$ Patty Miller, ${ }^{1}$ Ivan Lizarraga, ${ }^{1}$ John Goff, ${ }^{1}$ Carl Taganas ${ }^{1}$

\section{${ }^{1}$ Kaiser Permanente Southern California}

Background/Aims: A family history of colorectal cancer (CRC) and/or endometrial cancer, especially under age 50, is suggestive of Lynch syndrome, the most common form of inherited colorectal cancer. Limited family history (FH) data are currently collected on our members by nongenetics providers and recorded in the FH tab of our electronic medical record (EMR). We used these data as a potential "screening tool" for inherited CRC.

Methods: We queried our EMR (Epic, Verona, WI) system to identify individuals meeting the following criteria: 1) age 25-50 years, 2) no personal history of CRC, 3) FH of at least two relatives with $\mathrm{CRC}$, and 4) accessed our electronic patient portal at least once in the last year. We identified 444 members who met all criteria and had not already had a genetics consultation. All received a secure email requesting additional personal and $\mathrm{FH}$ via a secure electronic questionnaire (created by using HP Process Automation). Survey respondents provided information on: a) known mutation in a cancer susceptibility gene in the family, b) FH of colon, endometrial and other cancers, and c) personal/FH of polyps. Based on their responses, members were informed that: a) genetics would schedule a consultation to discuss their FH, or b) they do not appear to be in increased risk for a cancer syndrome.

Results: At present 106 (24\%) of those contacted completed the survey, and 96 (91\%) of them meet our moderate/highrisk criteria. Over half have been scheduled/seen and genetic testing is in progress for some. Males and Hispanic/Latino members were underrepresented in both the original cohort and in the group of respondents. Non-Hispanic Caucasians were overrepresented in both groups.

Discussion: The underrepresentation of males and Hispanics in both the original cohort and the group of respondents suggests that gender and racial/ethnic background play a role in the likelihood of the patient reporting a FH of cancer. The high proportion of high-/moderate-risk responses suggests that those with more suspicious histories may be more motivated to complete the survey. Our results will help us determine if mining limited FH data in the EMR is an effective method for identifying inherited CRC syndromes.

Keywords: family history colorectal cancer, data mining electronic medical record

PD2-7:

Use of Imaging for Staging of Localized Breast Cancer in Two Integrated Health Care Systems: Adherence to a Choosing Wisely Recommendation

Erin E. Hahn, ${ }^{1}$ Tania Tang, ${ }^{1}$ Janet S. Lee, ${ }^{1}$ Corrine Munoz-Plaza, ${ }^{1}$ Joyce O. Adesina, ${ }^{1}$ Ernest Shen, ${ }^{1}$ Braden Rowley, ${ }^{2}$ Jared L. Maeda, ${ }^{3}$ David M. Mosen, ${ }^{4}$ John C. Ruckdeshel, ${ }^{2}$ Michael K. Gould ${ }^{1}$

\section{${ }^{1}$ Kaiser Permanente Southern California; ${ }^{2}$ Intermountain Healthcare; ${ }^{3}$ Mid-Atlantic Permanente Research Institute; ${ }^{4}$ Kaiser Permanente Northwest}

Background/Aims: The initial American Society of Clinical Oncology "Top 5" list, created as part of the Choosing Wisely campaign, recommends against use of imaging for staging of localized breast cancer in asymptomatic women at low risk for metastasis. The objective of this study was to measure and compare use of imaging for staging in two large integrated health care systems, Kaiser Permanente (KP) and Intermountain Healthcare (IH). We also sought to distinguish whether imaging was used for routine staging or for diagnostic purposes.

Methods: We identified stage 0-IIB breast cancer patients diagnosed between January 1, 2010, and December 31, 2012, with first primary malignancy from tumor registries in three KP regions (Southern California, Northwest, and Mid-Atlantic) and $\mathrm{IH}$. Using the KP and $\mathrm{IH}$ electronic health records, we identified use of imaging tests (positron emission tomography, computerized tomography [CT], bone scan) during the staging window, defined as 30 days prior to diagnosis up to initial cancer-directed surgery. We performed chart abstraction on a stratified random sample of patients who received an imaging test to identify clinical indication. 
Results: For the total sample of 10,014, mean age at diagnosis was 60 years (range $22-99$ ), with $21 \%$ stage $0,47 \%$ stage I and $32 \%$ stage II. Overall, $8 \%$ of patients $(n=792)$ received at least one imaging test during the staging window, including $8 \%$ at $\mathrm{KP}$ and $6 \%$ at $\mathrm{IH}(\mathrm{P}=0.0005)$. The most commonly used service was CT, with $82 \%$ of imaging services. Chart abstraction $(n=129)$ revealed that overall, almost half of all imaging tests (48\%) were performed to evaluate a symptom, sign or prior imaging finding, including 55\% at KP and $32 \%$ at IH. Symptoms and signs included chest pain, weight loss and palpable masses.

Discussion: Use of imaging for staging of low-risk breast cancer was very low in both health care systems, with clinically trivial differences between them. Approximately half of imaging services were in response to a sign or symptom. Strategies to reduce use of imaging for staging of localized breast cancer within these health care systems are unlikely to yield meaningful improvement.

Keywords: breast cancer, diagnosis, staging

\section{PD2-8:}

Implementing a Multisite, Transdisciplinary CaseControl Study to Assess Effectiveness of Screening Colonoscopy for Preventing Death From Colorectal Cancer (SCOLAR)

Tracy A. Becerra, ${ }^{1}$ Virginia P. Quinn, ${ }^{1}$ Douglas A. Corley, ${ }^{2}$ Michael Goodman, ${ }^{3}$ Ann G. Zauber, ${ }^{4}$ Christopher D. Jensen, ${ }^{2}$ Alexis Zebrowski, ${ }^{5}$ Chyke A. Doubeni ${ }^{5}$

\section{${ }^{1}$ Kaiser Permanente Southern California; ${ }^{2}$ Kaiser Permanente Northern California; ${ }^{3} E$ mory University; ${ }^{4}$ Memorial Sloan-Kettering Cancer Center; ${ }^{5}$ University of Pennsylvania}

Background/Aims: Colorectal cancer (CRC) is the second leading cause of cancer death in the United States. There is a need for observational studies that assess effectiveness of "screening" colonoscopy by carefully defining indication for exam. "Screening for Colorectal Cancer in Average-Risk Adults (SCOLAR)," is a transdisciplinary study to determine if colonoscopy is as effective as believed, filling a critical void in the evidence on effectiveness of CRC screening interventions in reducing the risk of death in real-world practice.

Methods: We describe implementation of SCOLAR, an ongoing case-control study at Kaiser Permanente Northern and Southern California conducted by investigators from seven institutions. All subjects had 5 years minimum prior health plan enrollment, no personal or strong family history of $\mathrm{CRC}$, or diagnosis of inflammatory bowel disease. Cases are subjects who died from invasive colorectal adenocarcinoma (2006-2012) and were 55-90 years old on date of death. Controls were individually matched to cases on service area, sex, birth date and enrollment years. SAS programs were centrally developed and adapted locally for sampling patients and extracting electronic data to populate a standardized data collection tool. Data collection involves chart reviews of electronic medical records and paper charts to identify patient screening history for a minimum of 10 years prior to cancer diagnosis (or reference date for controls). In addition to data quality safeguards, i.e. inter-rater validation or teleconferences among chart abstractors, expert reviewers perform adjudication of indications to differentiate screening from diagnostic procedures, a key methodological strength to assess true CRC prevention.

Results: To date, 4,753 chart abstractions have been performed, of which $48.8 \%$ of subjects were women, 35.3\% were 55-64 years of age, $9.5 \%$ were Hispanic, and $70.9 \%$ were white. We found a high level of accuracy using electronic data to capture receipt of colonoscopy. We are currently performing reviews and adjudication of data to create indication. Preliminary results show that $43 \%$ had a screening fecal occult blood test within 2 years of the reference date, $39.7 \%$ had a flexible sigmoidoscopy, and $32 \%$ had at least one colonoscopy.

Discussion: We believe SCOLAR provides a model for overcoming methodological issues affecting case-control studies of the effectiveness of colorectal cancer screening.

Keywords: colorectal, cancer, screening

PD2-9:

Using Tumor Registry and Electronic Medical Record Databases to Determine Incidence of Uterine Sarcoma and Occult Malignancy Within an Integrated Health Care System

Ted E. Palen, ${ }^{1}$ Kimberly J. Bishoff, ${ }^{1}$ Lue-Yen Tucker, ${ }^{2}$ Tina R. Raine-Bennet ${ }^{2}$

${ }^{1}$ Kaiser Permanente Colorado; ${ }^{2}$ Kaiser Permanente Northern California

Background/Aims: There are currently no reliable estimates of uterine sarcoma incidence and occult malignancy at hysterectomy to aid decision-making about surgical route and policy formulation.

Methods: We identified women 18 years or older who underwent a hysterectomy and had a new diagnosis of uterine sarcoma in 2006-2013 in a large integrated health care system. Clinical presentation including when the diagnosis was not suspected or known preoperatively was assessed. Age- and race-specific total and annual incidence of uterine sarcoma and leiomyosarcoma and occult malignancy were calculated and evaluated for trends over time.

Results: From 2006 to 2013 there were 93,153 hysterectomies, of which 49,314 (52.9\%) were for leiomyoma or abnormal bleeding. There were 298 new cases of uterine sarcoma, more than half $(58 \%)$ of which were suspected or known preoperatively. The incidence of occult uterine sarcoma and leiomyosarcoma among women having hysterectomies for presumed benign leiomyoma and abnormal bleeding was 2.51 and 1.66 per 1,000 hysterectomies, respectively. The incidence was significantly higher for women over age 50; however, there was no significant difference in incidence by race/ethnicity. The annual incidence of uterine sarcoma did not increase over the 8-year period.

Discussion: Data from a large, diverse, community-based U.S. population confirms that uterine sarcomas are rare. The 
risk of occult malignancy must be weighed against the known benefits of minimally invasive hysterectomy. Our estimates of the risk of occult malignancy add important information for discussions between patients and clinicians when considering minimally invasive surgery and power morcellation and providing informed consent.

Keywords: leiomyosarcoma, hysterectomy

PS1-1:

\section{Quality Measures in Early Stage Oral Cavity Squamous Cell Carcinoma}

\section{Steven S. Chang ${ }^{1}$ \\ ${ }^{1}$ Henry Ford Health System}

Background/Aims: Head and neck cancer treatment practices and outcomes vary from institution to institution. Other than survival data, there has been no established method to evaluate an institution's quality of cancer care. To address this issue, the American Head and Neck Society (AHNS) developed quality guidelines for head and neck cancer care based on systematic literature review and expert opinion. These guidelines represent critical aspects of accepted standards of oral cancer care, and the presence or absence of these measures is most likely to affect the overall outcome regardless of patient comorbidity. The purpose of this study is to evaluate the quality of oral tongue cancer care at our institution and validate these established quality measures.

Methods: A retrospective chart review of 73 patients treated at Henry Ford Health System with stage I-III oral tongue squamous cell carcinoma from 1991 to 2012 was performed, using 26 clinical measures based on AHNS quality guidelines. Correlations between parameters and endpoints were assessed by the Pearson chi-square test or, when there were $<10$ subjects in any cell of a $2 \times 2$ grid, by the two-tailed Fisher exact test.

Results: Of the 26 measures, 4 major measures were established. These were postoperative TNM staging $(95.2 \%$ compliance rate), documentation of margin status in pathology reporting (93.6\%), appropriate referral for radiation postoperatively based on NCCN guidelines (96.8\%), and neck dissection based on $>4 \mathrm{~mm}$ depth of invasion of the primary (performed in $84.5 \%$ of the qualifying patients). Overall 5-year survival for patients evaluated before 2007 was 58\%. Of the 26 clinical endpoints, a majority of demographic and social history information was present with a compliance of $95 \%$. However, other clinical endpoints such as preoperative speech pathology evaluation or dental evaluation had compliance rates of $<20 \%$.

Discussion: We were able to successfully review our own practice, validate the AHNS quality measures and identify areas that require improvement. This provides support to the validity of the major measures of quality head and neck cancer care established by the AHNS.

Keywords: head and neck cancer, quality

\section{Abstract \#PS1-6 withdrawn by authors}

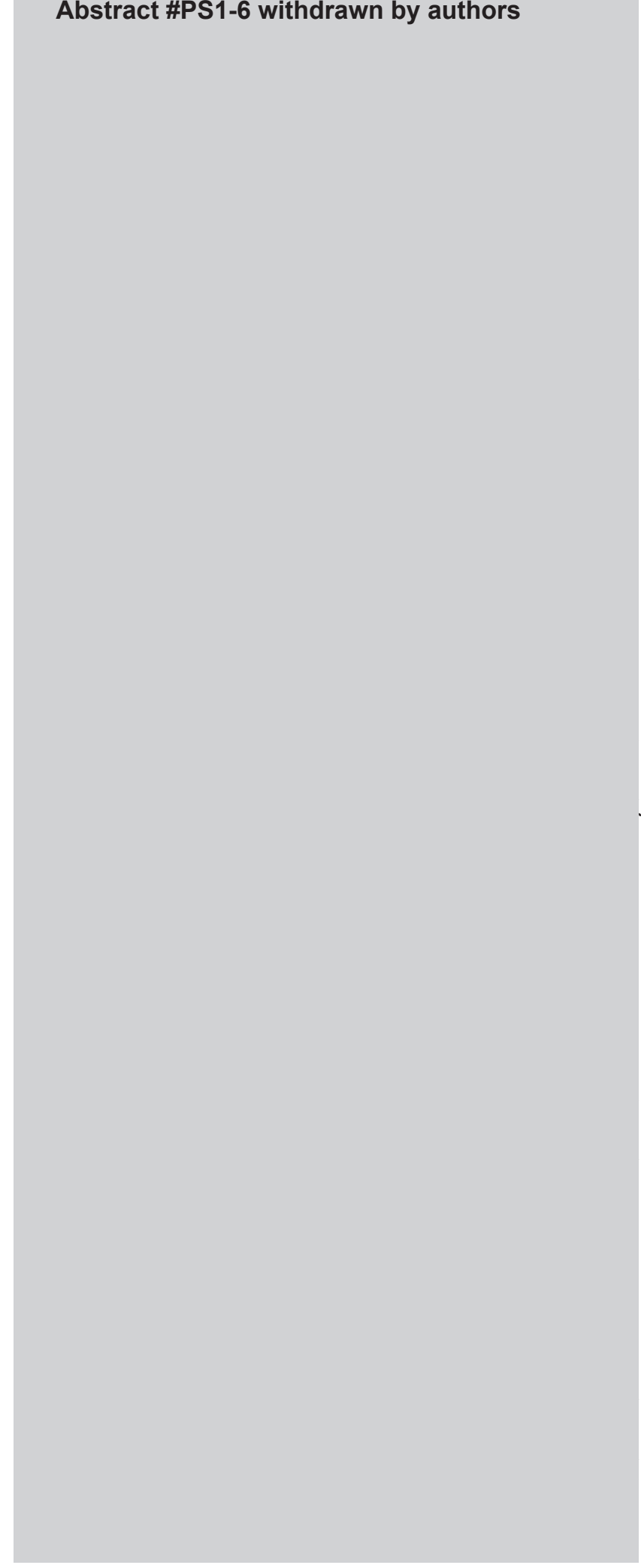

PS1-7:

Applying a Neutropenia Risk Model to Cancer Patients Using VDW Data: A CRN Pilot Study 
Pamala A. Pawloski, ${ }^{1}$ Lois E. Lamerato, ${ }^{2}$ Diana S. Buist, ${ }^{3}$ Avis J. Thomas, ${ }^{1}$ Sheryl M. Kane,${ }^{1}$ Robert Rasmussen, ${ }^{2}$ Sharon Fuller ${ }^{3}$

\section{${ }^{1}$ HealthPartners; ${ }^{2}$ Henry Ford Health System; ${ }^{3}$ Group Health Cooperative}

Background/Aims: Neutropenia, an extreme reduction in infection-fighting white blood cells, is a dangerous side effect of cytotoxic chemotherapy for cancer treatment, especially when accompanied by fever. Neutropenia and febrile neutropenia are associated with treatment delays, chemotherapy dose reductions, hospitalizations, antibiotic use, death and high costs. The granulocyte-colony stimulating factors (G-CSFs), filgrastim, pegfilgrastim and tbo-filgrastim, reduce the risk of neutropenia and febrile neutropenia; however, they are also associated with additional patient visits, side effects and high treatment-related costs. G-CSF use was found to be inconsistent with recommended clinical guidelines (overuse among those at low risk and underuse among those at high risk for neutropenia), thus making it part of the Choosing Wisely campaign. Individual patient factors are associated with increased risk for neutropenia and febrile neutropenia beyond that of the prescribed chemotherapy. No study has investigated the effect of providing medical oncologists with neutropenia risk predictions in real time to optimize the use of G-CSF and reduce neutropenia/febrile neutropenia outcomes among patients. The ongoing work will assess the feasibility of applying an existing patient neutropenia risk model developed by Lyman et al. to existing electronic data files. This work builds upon feasibility work currently being conducted at HealthPartners Institute, Minneapolis, MN, and adds two more CRN sites (Group Health Research Institute, Seattle, WA, and Henry Ford Health System, Detroit, MI).

Methods: We will investigate the applicability of an adapted risk model to readily available electronic data (excluding clinical oncology treatment modules). We will adapt Lyman's model to work with available data, provide external validation and calibrate the adapted model for our patient population.

Results: To date, we have obtained IRB approval to conduct the study and are developing a distributed modular program for extraction of clinical and administrative data variables available in the HMORN's virtual data warehouse.

Discussion: This presentation will report preliminary data on neutropenia and febrile neutropenia rates, chemotherapy treatment variations and G-CSF use among racially and geographically diverse sites. The current CRN study will provide a necessary foundation for a planned external proposal designed to test and implement a real-world risk assessment tool intended for use by clinicians at the point of care.

Keywords: neutropenia, risk model

\section{PS1-9:}

Adaptive Resources and Unmet Needs Related to Ostomy Self-Care Among Long-Term Rectal Cancer Survivors

Joanna Bulkley, ${ }^{1}$ Carmit $\quad$ K. McMullen, ${ }^{1}$ Mark C. Hornbrook, ${ }^{1}$ Marcia Grant, ${ }^{2}$ Christopher Wendel, ${ }^{3}$ Lisa J. Herrinton, ${ }^{4}$ Robert S. Krouse ${ }^{5}$
${ }^{1}$ Kaiser Permanente Northwest; ${ }^{2}$ City of Hope; ${ }^{3}$ Southern Arizona Veterans Administration Health Care System; ${ }^{4}$ Kaiser Permanente Northern California; ${ }^{5}$ University of Arizona College of Medicine

Background/Aims: Surgical treatment for rectal cancer can result in an intestinal ostomy that requires lifelong adaptation and investment of physical, cognitive and financial resources. However, little is known about the prevalence of functional limitation related to ostomies among long-term cancer survivors.

Methods: In 2010-2011, we mailed questionnaires to 313 long-term rectal cancer survivors with ostomies $(\geq 5$ years postdiagnosis) from two Kaiser regions, Northern California and Northwest. Potential participants (KP members age $\geq 18$ years) were identified through tumor registries. The response rate was $66 \%(183 / 277)$. Data from the electronic medical record included hernia diagnoses and comorbidity score.

Results: Of respondents with ostomy who reported wearing a pouch (not irrigating), 64\% (103/160) were male. Mean age at survey was 75 years. Health status: Health problems that could affect ostomy care capacity were fairly common. Mean body mass index (BMI) at time of survey was 26.76 (standard deviation 5.42), with 70\% (105/150) of respondents reporting a significant change (+/-) in BMI since time of surgery; $28 \%$ $(45 / 160)$ of respondents had a hernia diagnosis at some time after their surgery. Adaptive resources: Of participants who reported paying for their ostomy supplies, 55\% (39/71) had at least some difficulty paying the cost. Women were significantly more likely to report difficulty than men $(\mathrm{P}=0.011)$. Fifteen percent $(23 / 155)$ reported receiving help with their ostomy care. While 63\% (100/160) of respondents were partnered at time of survey, women were significantly less likely to be partnered than men $(p<0.001)$. Functional limitations related to ostomy: $27 \%(39 / 147)$ of respondents reported changing their ostomy wafer or appliance more often than every 3 days, 27\% (41/154) had problems with ostomy leakage, $25 \%(39 / 155)$ had problems with the skin around the ostomy, and $21 \%(33 / 155)$ reported having difficulty caring for their ostomy.

Discussion: Most rectal cancer survivors have health conditions that can worsen impairment and undermine adaptive capacity related to ostomy care and report difficulty paying for their ostomy supplies. Approximately $25 \%$ of survivors experienced limitations across multiple domains of ostomy-related functioning, yet only $14 \%$ reported receiving assistance with ostomy care. Further research should explore whether/how gender and partnered status influence resources or unmet needs for ostomy care assistance.

Keywords: survivorship, ostomy

PS1-12:

Applying a Model to Predict Neutropenia Risk in Patients With Cancer Using Electronic Data

Pamala A. Pawloski, ${ }^{1}$ Avis J. Thomas, ${ }^{1}$ Sheryl M. Kane,${ }^{1}$ Diane B. Eggen, ${ }^{1}$ Laurie L. Van Arman ${ }^{1}$

${ }^{1}$ HealthPartners 
Background/Aims: Granulocyte-colony stimulating factors (G-CSFs) are indicated to decrease the incidence of infections associated with chemotherapy-induced neutropenia (a severe reduction in white blood cells) in cancer patients receiving myelosuppressive chemotherapy. Severe neutropenia or febrile neutropenia occurs in $25-40 \%$ of treatment-naïve patients and are associated with increased infection rates, treatment delays, dose reductions, hospitalizations, deaths and increased costs. In addition to the chemotherapy treatment regimen, patient-specific factors influence neutropenic risk. Current guideline recommendations do not include a mechanism to quantify patient risk when determining need to G-CSF support. Studies report over- and underuse of G-CSF among those at low and high risk for neutropenia. A neutropenia risk model developed by Lyman et al. showed prior chemotherapy, relative dose intensity (RDI), abnormal liver and renal function, and low white cell count are major risk factors. We are testing the feasibility of using readily available clinical and administrative data from the HealthPartners electronic medical record and Oncology Treatment Module to apply the Lyman model to data from adult cancer patients receiving first cycle chemotherapy.

Methods: We included breast, colorectal, lymphoid, lung and ovarian cancer patients to compare abstracted chart data to electronically extracted data. We will adapt the Lyman model to predict neutropenia risk using our database. We also will extract electronic data from our site's virtual data warehouse (VDW) for comparison with sites participating in a separate $\mathrm{CRN}$-funded pilot project to evaluate the feasibility of using VDW data to predict neutropenic risk.

Results: We identified and abstracted data on 235 patients who received one of 60 treatment regimens. The median age at diagnosis was 61 (range 30-86) and 32\% were male. Of those, $92(39 \%)$ received G-CSF. The most commonly administered chemotherapy included cyclophosphamide, etoposide, 5-fluorouracil and doxorubicin. Manually abstracted data will be used to validate the electronically extracted data. Data validation, including inter-rater reliability and RDI, is ongoing.

Discussion: G-CSF use among chemotherapy patients can be identified in electronic oncology treatment records. Validation of key variables to determine the feasibility of using electronic data for risk determination is ongoing.

Keywords: neutropenia, risk model

\section{PS1-13:}

Ductal Carcinoma in Situ Treatment DecisionMaking and Patient-Reported Outcomes: A Review of the Literature

\section{Cecilia Portugal, ${ }^{1}$ Albert J. Farias, ${ }^{1}$ Erika L. Estrada, ${ }^{1}$ Janet Mora Marquez, ${ }^{1}$ Aniket A. Kawatkar ${ }^{1}$ \\ ${ }^{1}$ Kaiser Permanente Southern California}

Background/Aims: The treatment for ductal carcinoma in situ (DCIS) is similar to invasive breast cancer despite evidence of low 10-year recurrence rates and mortality. Patient preferences and involvement with decision-making should play a key role in treatment decisions; however, little are known about how decision-making about the type of treatment influences patient-reported outcomes. The objective is to review the recent literature to determine how decisionmaking for treatment of DCIS influences patient-reported outcomes and to make recommendations on future studies to improve decision-making practices.

Methods: A search of PubMed database was conducted for manuscripts published 2009-2014. Search terms included "ductal carcinoma in situ" and "satisfaction with treatment" $(n=33)$, "decisional conflict" $(n=1)$ or "patient decision making" ( $n=137)$. Only articles that included patient-reported decision-making were included in the review. Articles were excluded if they aggregated patients with invasive or metastatic breast cancer.

Results: There were a total of 11 articles included in the review. We found that decisional conflict among patients was common. Physicians play an important role in women understanding the disease; however, there is controversy about treatment of DCIS among experts. Decisional conflict was related to a lack of knowledge about the disease, which led to confusion, anxiety, fear and depression. The studies showed that conflicting messages from health care professionals negatively impacted patient's abilities to make informed treatment decisions and patient-reported outcomes. In terms of health disparities, one study found that a lack of understanding about DCIS was greater in women from nonEnglish speaking backgrounds. Recommendations for future research include: 1) improve knowledge of DCIS and its complexities among health care professionals and patients, 2) address knowledge gaps by developing and testing the efficacy of "decision aids" to improve patients comfort in making informed treatment choices, 3) evaluate why various messages about DCIS exist among health care professionals, and 4) address the need to establish consensus on guidelines. Discussion: The literature suggests that patients often have a lack of knowledge and experience adverse psychological outcomes associated with a diagnosis of DCIS. More research is needed to improve informed treatment decisions about the disease for patients diagnosed with DCIS.

Keywords: DCIS, treatment, decision-making

PS1-16:

The Effects of a Nonclinical Caregiver's Presence When a Cancer Patient Is Receiving Highly Emetogenic Chemotherapy

\section{Marisa P. Stevens ${ }^{1}$ \\ ${ }^{1}$ Kaiser Permanente Southern California}

Background/Aims: A cancer diagnosis not only affects the patient but also affects the patient's family and friends. The primary treatment of cancer is chemotherapy. Nausea is the side effect of chemotherapy most patients fear. The study aimed at examining another aspect of helping patients cope with chemotherapy side effects.

Methods: Medical records of patients currently receiving chemotherapy were reviewed to determine eligibility. 
Participants had to be able to speak, read and understand English, and their chemotherapy protocol must have included at least one highly emetogic-classified chemotherapy drug from the following: Carboplatin, Cytoxan, Adriamycin or Cisplatin. Forty-one participants agreed to be part of the study. Participants were classified into two groups. Group A included 23 participants who had a family or friend caregiver present during their treatment session. Group B included 16 participants who were alone during their treatment session. Twenty-four hours postchemotherapy the participant was asked to rate their nausea on a 5 -point Likert scale $(0=$ none, 4=symptoms associated with life-threatening consequences). They also were asked to report if they had taken any antinausea medication (Compazine), and specifically how many pills in the last 24 hours. Seventy-two hours postchemotherapy the participant was asked to complete the Functional Living Index-Emesis; responses to each question were on a 7-point Likert scale.

Results: The study examined the possibility of a relationship between a nonclinical caregiver's presence, their level of nausea symptoms and their quality of life. The study showed that a nonclinical caregiver's presence did not affect the patient's symptoms of nausea or their need to take less antinausea medications. The study supports the idea that a cancer patient's quality of life can be improved by the presence of a nonclinical caregiver during a patient's treatment session.

Discussion: The study's findings suggest there is a benefit to an improved quality of life if a cancer patient receiving highly emetogenic chemotherapy medication has a nonclinical caregiver with them during the time of administration. Although, cancer patients having a nonclinical caregiver present at their side while they receive chemotherapy seems to have no effect on a patient's reported nausea levels.

Keywords: caregiver, chemotherapy

\section{PS2-39:}

\section{Developing an Algorithm to Identify History of Cancer Using the Electronic Medical Record}

Christina L. Clarke, ${ }^{1}$ Heather S. Feigelson, ${ }^{1}$ Michelle G. Wrenn ${ }^{1}$

\section{${ }^{1}$ Kaiser Permanente Colorado}

Background/Aims: In studies of cancer incidence, it is often desirable to exclude individuals with any prior cancer. While the virtual tumor registry (VTR) can be a valuable tool in identifying cancer cases, these data may be incomplete. In particular, cancers diagnosed prior to health plan enrollment, or diagnosed out of network, may not be captured in the VTR. Further, data in the VTR may lag several months behind, so recently diagnosed cases may be missed. As part of a new study, we needed to identify cancer-free adults in the current Kaiser Permanente Colorado member population. To accomplish this, we developed an algorithm using our electronic medical record (EMR) data to identify individuals who have never had cancer.

Methods: Kaiser Permanente Colorado members between the ages of 40-75 years who were continuously enrolled during
2013 were included. We used administrative and EMR data, including the VTR, chemotherapy files, and inpatient and outpatient claims, to identify those with a high likelihood of prior cancer.

Results: A total of 203,888 members met our initial inclusion criteria. The algorithm flagged 31,885 (15.6\%) people as having a history of cancer. Of these, 10,494 (33\%) cancer cases were identified by the VTR, 9,125 (29\%) were identified with only a diagnosis code of cancer, and 7,812 (25\%) had only a receipt of chemotherapy. Having at least 2 visits to oncology accounted for $1,324(4 \%)$ cases. The remaining 3,130 (10\%) cases were a combination of the aforementioned categories. Most cases, 29,126 (91\%), were identified as having cancers prior to 2013. Of those with prior cancer, 61\% were women. Those flagged as having cancer were significantly older, with mean age 61 (9.2 standard deviation) compared to 56 (9.4 standard deviation) for those without cancer $(\mathrm{P}<0.0001)$. Chart reviews for 100 patients identified as not having cancer are underway to determine the algorithm's specificity.

Discussion: It is important to consider data sources outside the VTR to capture all health plan members with a history of cancer. Casting a wide net will help ensure that anyone with a history of cancer, whether diagnosed recently or prior to joining the health plan, will be identified.

Keywords: cancer, tumor registry

\section{CARDIOVASCULAR DISEASE}

\section{C1-3: \\ Lipid Management in Youth: What Are the Screening and Treatment Trends Within the Pediatric Cardiovascular Research Network?}

Jamie L. Webb, ${ }^{1}$ Chanin Wright, ${ }^{1}$ Debra Tan, ${ }^{2}$ Sean Gregory, ${ }^{2}$ Catherine J. McNeal, ${ }^{1}$ Laurel A. Copeland, ${ }^{1}$ Justin P. Zachariah, ${ }^{3}$ Andrea E. Cassidy-Bushrow, ${ }^{4}$ Jeffrey $\mathrm{O}$. Tom, ${ }^{5}$ Connie Mah Trinacty, ${ }^{6}$ Jeffrey $\mathrm{J}$. VanWormer, ${ }^{7}$ Eric A. Wright ${ }^{8}$

${ }^{1}$ Scott \& White Health System; 2 Texas A\&M School of Public Health; ${ }^{3}$ Boston Children's Hospital; ${ }^{4}$ Henry Ford Health System; ${ }^{5}$ University of Hawaii; ${ }^{6}$ Kaiser Permanente Hawaii; ${ }^{7}$ Marshfield Clinic Research Foundation; ${ }^{8}$ Geisinger Health System

Background/Aims: Atherosclerotic cardiovascular disease (ASCVD) accounts for 1 in every 3 deaths in the United States, and studies show that the precursors of atherosclerosis are apparent early in life. The correlation between childhood cardiovascular risk factors and the extent of atherosclerosis in adulthood has led to the development of guidelines aimed at attenuating ASCVD risks in youth. One major ASCVD risk factor is dyslipidemia, including both familial hypercholesterolemia $(\mathrm{FH})$ and lifestyle-related hyperlipidemia. Currently, few studies have investigated temporal lipid screening and treatment trends in youth and guideline adherence before and after the 2011 update, which includes universal lipid screening and targeted screening recommendations. The purpose of this study is to identify 
lipid screening and treatment trends and guideline adherence in a diverse population of children and adolescents across multiple health care systems participating in the Pediatric Cardiovascular Risk Network (P-CVRN) study. This data is also essential to provide up-to-date cost-effective analyses (CEA).

Methods: Institutional Review Board approval was obtained for this study. This retrospective database study includes $>$ 600,000 youth age 2 to 21 years with at least one outpatient visit captured across five P-CVRN sites between 2001 and 2013. The following de-identified data was captured and extracted at an individual patient level from inpatient and outpatient claims data: patient demographics, diagnoses, pretreatment laboratory values, initial lipid-lowering medications and doses, initial posttreatment laboratory values and presence of other risk factors or conditions.

Results: The data collected was used to analyze trends in pediatric lipid management and inform contemporaneous CEA related to pediatric lipid screening and treatment. Our data revealed that screening rates declined at most sites and varied from a peak of $\sim 25 \%$ to a low of $\sim 5 \%$ of youth. Lipid-lowering medications were prescribed in $0.02-0.19 \%$ of all youth, and prescribing did not generally increase over time. Lipid-lowering medication and dose initiation, pre- and posttreatment laboratory values, and medication possession ratio were analyzed using logistic regression. CEA did not support the benefit of universal lipid screening in youth.

Discussion: Current screening trends indicate that health care providers are nonadherent with guideline recommendations, and CEA reveals that universal screening in youth is not costeffective.

Keywords: lipid management, youth

PS1-5:

Cardiovascular Toxicity Following Aromatase
Inhibitor Use in 13,273 Women Cared for in an HMO

Reina Haque, ${ }^{1}$ Joanne E. Schottinger, ${ }^{1}$ Jiaxiao Shi, ${ }^{1}$ Joanie Chung, ${ }^{1}$ Chantal Avila, ${ }^{1}$ Britta Amundsen, ${ }^{1}$ Rowan T. Chlebowski

${ }^{1}$ Kaiser Permanente Southern California; ${ }^{2}$ University of California Los Angeles Medical Center

Background/Aims: Aromatase inhibitors (AIs) reduce breast cancer incidence in primary prevention trials (MAP3, IBIS2). However, controversy regarding AI's influence on cardiovascular disease (MI, angina and cardiac failure) (Amir et al., J Natl Cancer Inst., 2011) could limit use in prevention settings.

Methods: We assembled a cohort of 13,273 postmenopausal breast cancer patients initially cardiovascular disease (CVD)free at diagnosis in a large managed care organization. Women were diagnosed 1991-2010, and followed through 2012. The outcome, CVD risk, was compared across endocrine treatments (AI, tamoxifen [TAM], both, or neither). Information on demographics, comorbidity (diabetes, hypertension, etc.) and covariate medications (antihyperlipidemics, antihypertensives and other CVD drugs) were available from electronic medical records. We conducted Cox models using time-dependent endocrine drug use variables adjusted for age, demographics, comorbidity, CVD drug use, cancer treatment, tumor characteristics and tumor laterality.

Results: Among the 13,273-patient cohort, postmenopausal women who used AIs exclusively had a similar risk of ischemic disease (hazard ratio [HR]: 0.97, 95\% confidence interval [CI]: 0.78-1.22) and stroke (HR: 0.97, 95\% CI: 0.70-1.33) versus those who used TAM only (HR: 1.00, reference). However, women who used AIs only had a higher risk of other CVD disease combined (congestive heart failure, cardiomyopathy, dysrhythmia, valvular dysfunction, pericarditis) (HR: 1.26, 95\% CI: 1.11-1.43) than those exposed to TAM only. The risk of other CVD disease was greater among women exposed to sequential TAM and AI treatment. The results are based on 3,711 CVD events occurring in 72,886 woman-years of follow-up. Based on a subset of 7,982 patients who underwent breast irradiation, the risk of CVD overall was greater among women who used AIs only and received left-sided irradiation (HR: 1.21, 95\% CI: 1.02-1.44).

Discussion: Results indicate that variation exists in the type of CVD events that occur in breast cancer patients receiving AIs compared to tamoxifen users. For example, the risk of ischemic disease or stroke was not elevated in those who used AIs only versus TAM users. However, overall CVD events were greater in women who used AIs only (or sequentially after TAM), especially if they received left-sided breast irradiation. While these observational study results require cautious interpretation, they provide a basis for comparing the benefits and risks of endocrine treatments.

Keywords: breast cancer, cardiovascular disease

\section{CARE IMPROVEMENT RESEARCH}

A1-1:

A Proactive Diabetes Panel Management Approach: Can It Work and How Does It Work in a Health Care Delivery System?

Anny H. Xiang, ${ }^{1}$ Mayra P. Martinez, ${ }^{1}$ Xinhui Wang, ${ }^{1}$ Anita P. Joshua, ${ }^{1}$ Joanie Chung, ${ }^{1}$ Michael Thai, ${ }^{1}$ Gail Lindsay, ${ }^{1}$ Michael Kanter, ${ }^{1}$ Steven J. Jacobsen ${ }^{1}$

\section{${ }^{1}$ Kaiser Permanente Southern California}

Background/Aims: Guidelines have been established for the care of type 2 diabetes (T2DM) patients. However, $\sim 50 \%$ of adult patients receive the recommended care. Factors contributing to the low rate may include shortage of primary care physicians (PCP) and lack of knowledge and compliance at the patient level. To close this care gap, Kaiser Permanente Southern California (KPSC) initiated proactive panel management (PPM). PPM is a standardized and centralized process in which a health care team supports physicians by proactively identifying and evaluating patients with key and actionable care gaps, and providing patientspecific recommendations to help close the gaps. Patients with glycated $\mathrm{HbA} 1 \mathrm{c} \geq 9 \%$ were eligible. The purpose of this study was to evaluate clinical comparative effectiveness of PPM in 
achieving $\mathrm{HbA} 1 \mathrm{c}$ control (defined as glycated $\mathrm{HbAlc}<9 \%$ ). Methods: Data for 10,581 adult T2DM patients who had glycated $\mathrm{HbA} 1 \mathrm{c} \geq 9 \%$ in 2011 (baseline) but had not received PPM in that year were extracted from electronic medical records. Logistic regression was used to evaluate effectiveness of PPM in 2012 (follow-up) on HbA1c control.

Results: Among the 10,581 patients, 6,728 (63.6\%) received PPM during 2012. At baseline, receivers were more likely to be 45-64 years of age, female, and had more years of education than nonreceivers. The two groups were similar in race/ethnicity and household income. During follow-up, the PPM group had a greater reduction in HbA1c (mean [standard deviation]: -2.0 [2.2] vs. -1.6 [2.1], $\mathrm{P}<0.0001)$ and a higher percentage of patients achieved HbA1c control $(63 \%$ [4,232 of 6,728$]$ vs. $54 \%$ [2,079 of 3,853$], \mathrm{P}<0.0001)$ than the non-PPM group. The differences were not explained by social demographics, baseline service utilization and HbAlc. More patients in the PPM group had a PCP visit and used an antidiabetic medication than the non-PPM group during 2012; these two factors explained the majority of the association between PPM and HbA1c control. PPM was most effective for patients with higher educational attainment, no PCP visit or use of any antidiabetic medication at baseline.

Discussion: PPM using patient-level information in electronic medical records and technology within a prepaid health care delivery system can effectively increase diabetic control for high-risk T2DM patients.

Keywords: diabetes, care improvement

\section{B1-6:}

Design and Implementation of a Clinician-Focused Intervention to Improve Diagnosis and Management of Symptomatic Vulvovaginal Atrophy: ClinicianReported Barriers

Kimberly K. Vesco, ${ }^{1}$ Joanna Bulkley, ${ }^{1}$ Amanda Clark, ${ }^{1}$ Kate Beadle, ${ }^{1}$ Ashley Borin, ${ }^{1}$ Michael Leo ${ }^{1}$

\section{${ }^{1}$ Kaiser Permanente Northwest}

Background/Aims: Nearly $50 \%$ of postmenopausal women experience symptoms related to vulvovaginal atrophy (VVA) including vulvovaginal dryness and irritation, painful intercourse, dysuria, urinary urgency and incontinence, and recurrent urinary tract infection. Effective treatment options are available, however few women seek treatment for these symptoms and few providers ask women if they are symptomatic. As part of the development of a clinicianfocused intervention to improve diagnosis and management of symptomatic VVA among Kaiser Permanente Northwest patients, we conducted a survey of primary care and $\mathrm{OB} /$ GYN clinicians to assess provider knowledge about VVA and barriers to its diagnosis and treatment.

Methods: We sent an email invitation to take a short online survey to all 353 Kaiser Permanente Northwest primary care and $\mathrm{OB} / \mathrm{GYN}$ clinicians with valid email addresses. The survey included VVA knowledge questions related to symptoms, diagnosis and treatment, and practice assessment questions. We asked clinicians to report all potential barriers to VVA diagnosis and treatment in their practice. A list of potential barriers and an open-ended response option were provided.

Results: The response rate was 34\% (120/353). Knowledge about VVA was good; 67\% (641/953) of responses were correct. Only $39.5 \%(47 / 119)$ of clinicians were likely to assess VVA in a postmenopausal patient if she did not mention symptoms. Most clinicians rated their confidence in counseling their patients as medium or less regarding menopause-related vaginal discomfort $(56.1 \% ; 67 / 118)$ and the risks/benefits of vaginal estrogen therapy $(58 \%$; 69/119). Commonly reported barriers were lack of time during the visit $(75.4 \% ; 86 / 114)$, lack of patient education materials $(45.6 \%$; $52 / 114)$ and clinical tools $(18.4 \% ; 21 / 114)$, patient discomfort with discussing vulvovaginal concerns (41.2\%; 47/114), warnings about risks of estrogen medication in elderly women $(36.8 \% ; 42 / 114)$, and the clinicians' lack of knowledge about VVA $(32.5 \% ; 37 / 114)$.

Discussion: Provider knowledge about VVA symptoms and treatment is generally good. Barriers to patient care such as time, lack of clinical support tools, and patient-provider discomfort with discussion of VVA may explain why this easily treated condition is frequently underdiagnosed and undertreated. Our second abstract describes the intervention phase of our study, which includes the design and implementation of a clinicianfocused intervention to improve diagnosis and management of symptomatic VVA.

Keywords: patient care improvement, postmenopause

B4-1:

Problematic Events in Critically III Patients: The Perspectives of Surrogate Decision-Makers

Kimberly A. Fisher, ${ }^{1}$ Sumera Ahmad, ${ }^{1}$ Madeline Jackson, ${ }^{2}$ Kathleen M. Mazor ${ }^{2}$

${ }^{1}$ University of Massachusetts Medical School; ${ }^{2}$ Meyers Primary Care Institute

Background/Aims: Critically ill patients are particularly vulnerable to breakdowns in medical care due to the severity and complexity of their illness. Although many critically ill patients are unable to communicate or participate in their own care, their surrogate decision-makers (SDMs) are likely to have important insights regarding preventable problematic events that may occur in the intensive care unit (ICU). We describe the perspective of SDMs of critically ill patients with such events.

Methods: We enrolled SDMs of adult critically ill patients with respiratory failure in any of the 7 ICUs at a single tertiary medical center in central Massachusetts. Participating SDMs were contacted by telephone approximately 4 weeks after the patient's discharge and screened for those who identify a problematic event. Problematic events are defined as something that "went wrong," that could have been prevented, and that had a deleterious impact on the patient or SDM. Participants who identify an event that meets the above criteria were invited to undergo an in-depth qualitative interview. 
Results: Of the 127 SDMs we approached for participation, $91(72 \%)$ agreed to participate, and complete follow-up is available on 70 of the 91 participants (77\%). Of those with complete follow-up, 43\% (30 of 70) identified at least one eligible problematic event, with many SDMs identifying multiple events. Problematic events included breakdowns in medical care (delay in diagnosis, misdiagnosis, delayed or inadequate treatment, preventable complications, and premature discharge) and major lapses in communication (insufficient information, inadequate access to health care providers, problems with communication regarding end-oflife preferences, and episodes of rude or dismissive treatment by health care providers). These problems had a significant negative impact on patients and their families leading to physical harm, prolonged hospitalization, rehospitalization, severe emotional distress, life disruption, and provision of medical care inconsistent with patients' wishes.

Discussion: SDMs frequently identify preventable problems in the care of critically ill patients with significant adverse consequences for both patients and SDMs. These findings highlight the importance of the perspective of SDMs of critically ill patients in understanding breakdowns in care and may identify targets for preventing such events.

Keywords: patient safety, qualitative

\section{B4-4:}

System Alignment for VaccinE Delivery (SAVED): Qualitative Interviews Inform a Technology-Based Intervention to Improve Influenza and Pneumococcal Vaccination Rates

Sarah L. Cutrona, ${ }^{1}$ Larry Garber, ${ }^{2}$ Lloyd Fisher, ${ }^{1}$ Peggy Preusse, ${ }^{3}$ Sarah L. Goff, ${ }^{4}$ Meera Sreedhara, ${ }^{2}$ Madeline Jackson, ${ }^{2}$ Devi Sundaresan, ${ }^{3}$ Kathleen M. Mazor ${ }^{2}$

${ }^{1}$ University of Massachusetts Medical School; ${ }^{2}$ Meyers Primary Care Institute; ${ }^{3}$ Reliant Medical Group; ${ }^{4}$ Baystate Medical Center

Background/Aims: Influenza and pneumococcal vaccines are beneficial but underutilized. Patient interviews may help identify effective outreach strategies and increase vaccination rates.

Methods: We conducted in-depth qualitative interviews with a purposive sample of adults whose primary care physicians were part of a large multispecialty group practice in central Massachusetts. Interviews occurred between April and October 2014, with individuals who were not up to date on either influenza or pneumococcal vaccines. The goal of the interviews was to: (a) understand barriers to influenza and pneumococcal vaccination, and (b) inform development of provider educational materials and patient outreach materials. Results: We interviewed 14 people, 57\% under age 65 (8 out of 14); 64\% female (9 out of 14) (additional interviews are in process). Major themes included trust, influence of family and friends, and impact of vaccination choice on others. Many participants trusted their doctor's recommendations but did not trust vaccine safety and efficacy. In some cases, the safety of influenza vaccines was compared unfavorably with other vaccines because "they make it up fresh each year" as compared to a vaccine where "it has been given for years." The majority of participants cited relationships of family, friends or coworkers as influencing their own vaccination decision. While some participants cited concern for others' health as a reason to get vaccinated ("it's not fair if you get sick and spread it to everybody else"), others voiced concerns that care-giving responsibilities could be compromised if the vaccine induced illness. Several participants had not previously heard of the pneumococcal vaccine. Preferred modes of information on vaccination included verbal and electronic; hard-copy handouts were preferred by slightly fewer patients. When asked about effective messaging, some responded unfavorably to message components perceived to be condescending or intrusive ("our records indicate that you haven't yet gotten this vaccine").

Discussion: We identified several potential barriers to vaccination for patients who were not up to date with influenza or pneumococcal vaccination. Based on these findings, we are developing patient messages that include references to protecting the health of friends and family but which minimize language implying that the sender has been monitoring vaccination behavior.

Keywords: flu vaccine, qualitative

B4-5:

An In-Depth Interview Study of Immunization Providers to Understand Practice Patterns, Barriers and Facilitators for Administering the 3-Dose HPV Vaccine Series

\section{Melissa Preciado, ${ }^{1}$ Sharon M. Hudson, ${ }^{1}$ June Rondinelli, ${ }^{1}$ Beth A. Glenn, ${ }^{2}$ Chun Chao ${ }^{1}$ \\ ${ }^{1}$ Kaiser Permanente Southern California; ${ }^{2}$ University of California Los Angeles}

Background/Aims: Vaccination with the quadrivalent human papillomavirus (HPV) vaccine presents an opportunity to reduce the burden of conditions caused by HPV types 6, 11, 16 and 18. Despite increases in HPV vaccine initiation since its introduction in 2006, the 3-dose series completion rate among age-eligible males and females remains low. Provider recommendation has been identified as one of the strongest influences on HPV vaccine initiation. However, limited research has examined the contribution of provider factors to HPV vaccine completion. The purpose of this study was to gain an understanding of barriers and facilitators to HPV vaccine series completion from the perspective of immunization providers.

Methods: Rates of HPV vaccination were examined for 201 pediatrics and family medicine clinics serving adolescent members in Southern California. These clinics were ranked with regard to the proportion of age-eligible males and females who completed the 3-dose HPV vaccine series in order to identify the three highest and three lowest performing clinics. Data were collected through semistructured telephone interviews within three subsets of immunization providers: pediatricians, family practice physicians, and immunization 
nurses. Our goal was to interview at least 10 providers from each subset within the highest and lowest ranked clinics in order to reach thematic saturation. Providers were recruited through presentations at staff meetings and via email and telephone. The Health Behavior Framework and the Competing Demands Model were used to develop the interview guide.

Results: Sixty-two interviews were conducted from December 2013 to August 2014; 30 providers were recruited from clinics with the lowest completion rates and 32 from the highest-performing clinics. Interviews were recorded and transcribed verbatim. Interviews focused on the following themes: 1) provider knowledge, attitudes and behaviors, 2) perceptions of patient knowledge and attitudes, and 3) system-level influence on vaccination. Data analysis using Nvivo software is ongoing. It is anticipated that final theme generation will be completed by December 2014. Additional results will be discussed.

Discussion: We expect that the results of this study will help guide the design of multilevel interventions targeting the provider and health care system to improve HPV vaccine series completion.

Keywords: HPV vaccine, adherence

\section{C1-5: \\ Evaluation of Knee Arthroscopy as a Low-Value Intervention for Treatment of Meniscal Damage in Patients With Osteoarthritis} Mary Helen Black, ${ }^{1}$ Ronald A. Navarro, ${ }^{1}$ Annette L.
Adams $^{1}$

\section{${ }^{1}$ Kaiser Permanente Southern California}

Background/Aims: Patients presenting to their physicians with knee pain are often evaluated for osteoarthritis (OA) and/or meniscal damage. To alleviate pain and delay/prevent more invasive knee surgery, meniscal damage is typically treated with knee arthroscopy (KA) even if mild/moderate $\mathrm{OA}$ is present. However, recent randomized trials suggest that KA for meniscal damage in the setting of OA does not result in improved physical functioning and/or greater pain relief than treatment with physical therapy (PT) alone. Given these data, our aims were: 1) determine the demographic/clinical patient factors that lead to treatment with KA or PT-only, and 2) assess differences in clinical outcomes for KA vs. PT-only. Methods: The cohort is comprised of patients aged $>45$ years, with $>2$ years membership, no prior knee surgery and recent history of OA at the time of meniscal tear diagnosis (20032006). Patients were followed from date of surgery (KA) or first PT visit (PT-only) until partial/total knee replacement surgery, death, disenrollment or end of study (12/31/2013), whichever occurred first. Cox proportional hazards and robust Poisson models were used to estimate the risk of knee replacement surgery and greater utilization/medication use, respectively, associated with KA vs. PT-alone. Demographic/ clinical characteristics were used to derive a propensity score, and inverse probability of treatment weights applied to individual observations in the estimation of model parameters.
Results: Among 7,026 patients (69\% KA, 31\% PT-only), $27 \%$ had partial/total knee replacement surgery during follow-up. PT-only patients were older and more likely to be female, have greater comorbidity and utilize health services more frequently than patients with KA. After accounting for differences between groups, KA patients were no better off than PT-only patients in terms of time to knee replacement surgery; cumulative incidence of knee surgery was significantly higher for those who received KA vs. PT-only (hazard ratio: 1.30, 95\% confidence interval: 1.16-1.46). No differences in utilization for knee pain, narcotic medication dispenses or knee injections were observed between the groups.

Discussion: For patients with meniscal damage complicated by $\mathrm{OA}$, treatment with $\mathrm{KA}$ does not result in better clinical outcomes than PT-alone. Considering Choosing Wisely and Kaiser Permanente organizational goals, reduction in KA procedures performed for these patients may be warranted.

Keywords: orthopedic surgery, comparative effectiveness

D3-1:

Value of Primary Care Diabetes Bundle Management: Long-Term Impact on Cost

\section{Daniel D. Maeng, ${ }^{1}$ Yan Xiaowei, ${ }^{1}$ Thomas R. Graf ${ }^{1}$}

\section{${ }^{1}$ Geisinger Health System}

Background/Aims: In 2006, Geisinger's primary care clinics implemented an all-or-none diabetes bundle system of care, which has been shown to be associated with reduced risk of retinopathy, stroke and myocardial infarction (Bloom et al., 2014). This follow-up study estimates long-term cost savings associated with patients' exposure to the all-or-none bundle of measures for primary care management of diabetes.

Methods: Claims data from Geisinger Health Plan were used to identify those who met Healthcare Effectiveness Data and Information Set criteria for diabetes and had two or more diabetes-related encounters on different dates before 2006 . A cohort of 2,426 members exposed to the diabetes bundle was then compared against a propensity score-matched comparison cohort of 1,836 members from January 1, 2006, through June 30, 2013. A set of generalized linear models with log link and gamma distribution was estimated. The key explanatory variable was each member's bundle exposure measured in month. The dependent variables were inpatient and outpatient facility costs, professional cost and total medical cost measured on a per-member-per-month (PMPM) basis.

Results: During the first year of exposure, the total cost of care including prescription drugs rose $1.5 \%(\$ 9 / \$ 604$; $\mathrm{P}=0.67)$ PMPM. Also during the first year of the diabetes bundle exposure, there were statistically significant increases in the outpatient $(12.6 \%[\$ 19 / \$ 150])$ and professional $(10 \%$ $[\$ 15 / \$ 153])$ PMPM costs $(\mathrm{P}<0.05)$. However, in the third and fifth years, there were approximately $10 \%(\$ 67 / \$ 666)$ and 16\% (\$118/\$764) PMPM total cost savings, respectively $(\mathrm{P}<0.05)$. Over the entire study period, the estimated total medical cost saving was $6.8 \%(\$ 44 / \$ 650 ; \mathrm{P}<0.05)$ PMPM. The main source of the savings was reductions in inpatient 
facility cost, which accounted for approximately half of the total cost reductions.

Discussion: A system of care with an all-or-none bundled measure used in primary care for patients with diabetes may reduce long-term cost of care while improving health outcomes.

Keywords: diabetes care, cost of care

\section{$\mathrm{ECl}-1$ : \\ Use of Low-Value Surveillance Services for Early Stage Breast Cancer Survivors}

Erin E. Hahn, ${ }^{1}$ Tania Tang, ${ }^{1}$ Janet S. Lee, ${ }^{1}$ Corrine Munoz-Plaza, ${ }^{1}$ Joyce O. Adesina, ${ }^{1}$ Ernest Shen, ${ }^{1}$ Braden Rowley, ${ }^{2}$ Jared L. Maeda, ${ }^{3}$ David M. Mosen, ${ }^{4}$ John C. Ruckdeshel, ${ }^{2}$ Michael K. Gould ${ }^{1}$

\section{${ }^{1}$ Kaiser Permanente Southern California; ' Intermountain Healthcare; ${ }^{3}$ Mid-Atlantic Permanente Research Institute; ${ }^{4}$ Kaiser Permanente Northwest}

Background/Aims: Guidelines from the National Comprehensive Cancer Network and the American Society of Clinical Oncology recommend against aggressive surveillance for early stage breast cancer survivors. However, breast cancer survivors commonly receive nonrecommended services, including advanced imaging and biomarker tests. The objective of this study was to measure use of posttreatment breast cancer surveillance services including carcinogenic antigen biomarker tests and advanced imaging in two integrated health care systems, Kaiser Permanente (KP) and Intermountain Healthcare (IH), and to identify potential target areas for de-implementation of low-value services.

Methods: We identified stage 0-IIB breast cancer patients diagnosed with first primary malignancy between January 1, 2009, and December 31, 2010, from tumor registries in three KP regions and all four IH regions. Using the KP and $\mathrm{IH}$ electronic health records, we identified use of advanced imaging tests (positron emission tomography [PET], computerized tomography [CT], bone scan) and biomarker tests over 18 months, starting one year after diagnosis. We excluded services performed in the emergency department or inpatient setting. We performed chart abstraction on a stratified random sample of patients who received an imaging or biomarker test to identify clinical indication: diagnostic (used in response to a clinical sign or symptom) or surveillance.

Results: Of the total sample of 7,217 patients, $23 \%$ received at least one imaging test during the surveillance period, including $25 \%$ at $\mathrm{KP}$ and $20 \%$ at $\mathrm{IH}(\mathrm{P}<0.05)$, and $27 \%$ of patients received at least one biomarker test, $36 \%$ at KP and $12 \%$ at $\mathrm{IH}(\mathrm{P}<0.05)$. Chart abstraction $(\mathrm{n}=155)$ revealed that the majority of imaging services $(90 \%)$ were diagnostic, while the majority of biomarker (96\%) tests were used for surveillance in absence of documented signs or symptoms.

Discussion: There is substantial use of imaging services and biomarker tests during posttreatment care of early stage breast cancer patients. Review of health records revealed that the majority of imaging services were performed in response to clinical signs or symptoms, while the overwhelming majority of biomarker tests were performed for surveillance. Next steps include developing a tailored strategy that incorporates input from oncology clinicians to reduce use of surveillance biomarker tests as a care improvement project.

Keywords: breast cancer surveillance, low-value services

\section{PD1-1: \\ Providing an Upstream Discussion in the Ambulatory Setting to Assist Chronic Kidney Disease Patients Considering Dialysis}

Craig M. Nelson, ${ }^{1}$ Tuan K. Le, ${ }^{1}$ Mi Chang, ${ }^{1}$ Pushkar Chand, ${ }^{1}$ Julie Sortais, ${ }^{1}$ Karen Tallman ${ }^{2}$

\section{${ }^{1}$ Kaiser Permanente Southern California; ${ }^{2}$ Kaiser Permanente Northern California}

Background/Aims: Extensive discussion with renal patients about treatment intensity is not systematically integrated into their care. These discussions are often time-consuming, involve family, and require multiple discussions. The discussion often takes place during an acute hospitalization. We conducted a "test of change" pilot to assess the utility of providing an upstream discussion in the ambulatory setting as an additional nephrology consult to assist renal patients with chronic kidney disease considering treatment choice. We believed our pilot would show this additional nephrology consult would help our chronic kidney disease patients to be more informed about choosing or not choosing dialysis, and would also assist us in resource stewardship.

Methods: We randomly assigned nephrology patients at Kaiser Permanente South Bay Medical Center with stage 4 or 5 chronic kidney disease who had not yet begun dialysis. The test group received the additional nephrology consult and was seen by an interdisciplinary team comprised of a nephrologist, a social worker and a clinical ethicist.

Results: Those who received the additional nephrology consult experienced help in forming a treatment plan, felt well-understood, and had the opportunity to thoroughly discuss questions. The control group that did not receive the additional nephrology consult had a $26 \%$ increased probability of beginning dialysis, had a statistical increase for dialysis and clinic visits $(\mathrm{P}<0.10$ and $\mathrm{P}<0.05)$, and were more likely to be admitted to the hospital (control 0.5 vs. test 0.2 admissions per patient), spend more days hospitalized (control $2.8 \mathrm{vs.}$. test 0.5 bed days per patient), and be seen in the emergency room (control 0.73 vs. test 0.66 visits per patient) and clinic (control 6.6 vs. test 3.6 visits per patient).

Discussion: We attempted to review the utility of providing an upstream discussion in the ambulatory setting for renal patients considering treatment plan decision-making. Interdisciplinary consultations in the ambulatory setting allows us to begin a helpful conversation that can include helping patients and families better understand the possibilities of medicine, patients' treatment goals, and lived values from the patients' context.

Keywords: advance care planning, shared decision-making 
PD1-4:

Minimizing Medication Histories Errors for Patients Admitted to the Hospital Through the Emergency Department: A Three-Arm Pragmatic Randomized Controlled Trial of Adding Admission Medication History Interviews by Pharmacists or PharmacistSupervised Pharmacy Technicians to Usual Care

Joshua M. Pevnick, ${ }^{1}$ Caroline B. Nguyen, ${ }^{1}$ Cynthia A. Jackevicius, ${ }^{2}$ Katherine A. Palmer, ${ }^{1}$ Rita Shane, ${ }^{1}$ Catherine Bresee, ${ }^{1}$ Mackenzie E. Bear, ${ }^{1}$ Olga Zaitseva, ${ }^{1}$ David Seki, ${ }^{1}$ Anish Desai, ${ }^{1}$ Brian Doyle, ${ }^{3}$ Douglas S. Bell ${ }^{3}$

\section{${ }^{1}$ Cedars-Sinai Health System, ${ }^{2}$ Western University of} Health Sciences; ${ }^{3}$ University of California Los Angeles

Background/Aims: Meta-analysis shows hospitalized U.S. patients suffer 106,000 fatal adverse drug events (ADE) annually. Errors in admission medication histories (AMH) frequently cause preventable ADEs. There is also concern that electronic health records (EHR) propagate AMH errors into ADEs by making AMHs easily orderable. We hypothesized that for patients with complex AMHs, pharmacist-supervised pharmacy technician (PSPT) and pharmacist AMH interviews would reduce AMH errors.

Methods: At a large university-affiliated hospital, we conducted an IRB-approved, pragmatic randomized trial with informed consent waived. Inclusion criteria, accessed via EHR, were: $\geq 10$ chronic prescription medications, history of acute myocardial infarction or congestive heart failure, admission from skilled nursing facility, history of transplant, or active anticoagulant, insulin or narrow therapeutic index medications; patients were excluded if admitted to pediatric or trauma services or transplant services with pharmacists. All arms received usual care for patients admitted from the emergency department. This included registered nurses obtaining AMHs, and natural variation in checking, correcting and ordering from these AMHs by admitting providers. In the two intervention arms, PSPTs and pharmacists obtained initial AMHs. They contacted family, pharmacies and/or providers to resolve questions. As per prior studies, we obtained reference standard AMHs from all patients (usually one day after admission), and initial AMH errors were independently rated by $\geq 2$ pharmacists as significant, serious or life-threatening. Three error severities were assigned $-1,4$ and 9 points, respectively. We calculated weighted error scores for each patient, and mean scores/patient for each arm. We had $80 \%$ power to identify arms with higher/lower scores of $\geq 11.2$ / patient.

Results: Of 311 patients enrolled, 28 patients were later excluded (most not ultimately admitted, or discharged before reference standard AMH obtained). Median age and number of medications were 76 and 14 (interquartile ranges: 63-84 and 10-19), respectively. Patients in the usual care, pharmacist and PSPT arms had 7.4, 1.4 and 1.5 AMH mean errors/patient, respectively $(\mathrm{P}<0.0001)$; mean error scores were $21.2,3.9$ and 3.9 per patient, respectively $(\mathrm{P}<0.0001)$. Physician scoring of admission medication order errors due to AMH errors is ongoing.

Discussion: Pharmacists and PSPTs substantially reduced
AMH errors and weighted error scores, and thus show promise for reducing ADEs. Limitations include our single site, and using intermediate endpoints.

Keywords: adverse drug events, medication reconciliation

PD1-5:

Comparing Implementation Models Across Two Kaiser Permanente Regions to Improve Outcomes for Patients With Comorbid Depression and Diabetes

Mark Dreskin, ${ }^{1}$ Arne Beck, ${ }^{2}$ Gabrielle F. Beaubrun, ${ }^{1}$ Nancy E. Brace, ${ }^{2}$ Alisa Aunskul, ${ }^{1}$ Jennifer M. Boggs, ${ }^{2}$ Karen J. Coleman ${ }^{1}$

\section{${ }^{1}$ Kaiser Permanente Southern California; ${ }^{2}$ Kaiser Permanente Colorado}

Background/Aims: The Care for Mental, Physical, and Substance-use Syndromes (COMPASS) initiative was funded by the Centers for Medicare \& Medicaid Services (CMS) to implement collaborative care in primary care settings for patients with comorbid depression and diabetes, coronary artery disease and/or substance-use disorders. Here we compare implementation strategies in Kaiser Permanente Colorado (KPCO) and Southern California (KPSC) to inform potential dissemination strategies across other HMO sites.

Methods: COMPASS patients are enrolled if they have PHQ9 scores $>10$ and poorly controlled diabetes $(\mathrm{HbAlc}>$ 8.0). The proposed collaborative care model requires a team with a consulting psychiatrist, consulting physician and care manager. The care manager provides both behavioral health interventions and medical care, and facilitates a weekly structured case review to determine treatment intensification. The program lasts at least six months with six months of maintenance. COMPASS implementation at KPCO included a dedicated, centralized nurse care manager and therapist working together who telephonically outreached and followed patients. COMPASS implementation at KPSC used an existing depression care management program in primary care at four medical center service regions, requiring care managers to change their scope of practice to address diabetes. Both KPCO and KPSC held weekly structured case reviews. KPCO was centralized and KPSC was done by teams at each participating medical center.

Results: The initiative began in 2012. To date, KPCO has enrolled 303 patients and KPSC 712. Remission rates for depression are $26 \%$ in KPCO and 39\% in KPSC patients. Control rates for diabetics are $39 \%$ for $\mathrm{KPCO}$ and $38 \%$ for KPSC.

Discussion: Centralized care management may increase uniformity and fidelity of implementation, but limit reach and compromise outcomes without additional local primary care support. Conversely, adding care management tasks to existing staff may increase reach but attenuate outcomes without adequate staff training in co-management of psychological and medical care needs. Funded by the U.S. Department of Health and Human Services via CMS (\#1C1CMS331048-01-00), the contents of this publication are solely the responsibility of the authors and do not necessarily represent the official views of 
the U.S. Department of Health and Human Services or any of its agencies.

Keywords: collaborative care, depression

\section{PD1-6: \\ Medically Complex Pediatric Patient Care Model: Coordinated Team-Based Care With Supporting Health Information Technology to Implement Best Practices and Address Care Gaps of Transitioning Age Patients and Family Caregivers}

Susan R. Snyder, ${ }^{1}$ Thomas W. Davis, ${ }^{1}$ Rebecca A. Stametz, ${ }^{1}$ Deseraé N. Clarke, ${ }^{1}$ Amanda C. Bengier, ${ }^{1}$ Jonathan D. Darer ${ }^{1}$

\section{${ }^{1}$ Geisinger Health System}

Background/Aims: Medically complex pediatric patients have multiple severe chronic conditions consuming disproportionate health care resources. The top $1 \%$ of Geisinger's pediatric population uses health care representing $20 \%$ of total costs. Nationally, their needs are largely underserved by current delivery systems as they involve multiple specialists and settings, are challenging to coordinate, and place a tremendous burden on family caregivers. Providing effective care for children with special health care needs $(\mathrm{CSHCN})$ requires a system that is integrated, comprehensive, coordinated and family-centered to foster positive experiences between families and providers. Advancing integrated systems of care for CSHCN and their families is a national priority and reflected in the Healthy People goals. Geisinger has developed a new innovative approach to CSHCN starting with ages 15 and over: the Medically Complex Pediatric Patient (MCPP) care model. Its objective is to address patient and family caregiver needs along with drivers of poor quality and unnecessarily high costs of care. This approach includes: (1) a comprehensive care clinic with an enhanced professional care team to provide and coordinate health care supported by (2) care bundles for reliable delivery of best practices and (3) advanced health information technology using Geisinger's patient portal and web-based applications to efficiently document and facilitate timely care, planning, management and coordination and promote good communication between families and providers.

Methods: Development and implementation of the MCPP care model and an evaluation plan are occurring simultaneously. The evaluation plan begins with newly developed patient and family caregiver assessment questionnaires administered to all eligible families to collect baseline and postimplementation measures, with questions corresponding to multiple nationally validated surveys (National Survey of CSHCN, GAD-7, PHQ-9, Caregiver Strain Index).

Results: Survey results of approximately 300 families will establish intensity of caregiving needs, caregiver challenges and ability to meet needs effectively, service quality and gaps consistent with national measures including $\mathrm{CSHCN}$ core outcomes that facilitate integrated systems of care for CSHCN such as care coordination, partners in decisionmaking, and medical home to assess preimplementation gaps and postimplementation performance.

Discussion: Baseline results will be used to inform MCPP care model implementation and to assess its progress and performance over time consistent with national priorities.

Keywords: children with medical complexity, caregivers

PD1-10:
Bridging the Gap: Evaluating Osteoporosis Therapy
Medication Orders Versus Pharmacotherapy
Prescription Fills Following an Osteoporotic
Fracture

Jane R. Grafton ${ }^{1}$

\section{${ }^{1}$ Group Health Cooperative}

Background/Aims: Pharmacotherapy following osteoporotic fracture is a primary intervention strategy to prevent future fractures. Multiple effective pharmacotherapies are available, but postfracture treatment rates remain low. Osteoporosis treatment is defined as a prescription fill for oral or injectable bisphosphonate therapy. When a physician orders treatment but the patient does not fill, there is potential to address this gap by following up at patient level. If the physician does not order treatment, then appropriate intervention might be at physician level. This poster aims to identify gaps in treatment at Group Health Cooperative, whether they occur at patient or provider level.

Methods: The Post Fracture Care Study examines factors associated with nonreceipt of care following osteoporotic fracture between 2006 and 2012 in a cohort of 5,237 women aged $\geq 55$ years at fracture, with no osteoporosis pharmacotherapy in the 12 months preceding index fracture date. Information on treatment was captured from Group Health automated pharmacy data, which includes fills at Group Practice pharmacies as well as claims for fills at external pharmacies. Medication orders were considered if they occurred within 12 months on/after index fracture date. Treatment was operationalized as: a fill between 45 days prior to medication order and 45 days after order, and within 12 months after fracture date.

Results: $10.4 \%(543 / 5,237)$ were ordered a bisphosphonate; $12.3 \%(643 / 5,237)$ received treatment (filled an oral or injectable bisphosphonate); $0.8 \%(40 / 5,237)$ were ordered bisphosphonate but did not receive treatment; $2.7 \%$ $(140 / 5,237)$ received treatment, without record of order in EMR; and 87.0\% $(4,554 / 5,237)$ had neither medication order nor treatment.

Discussion: The gap of women with an osteoporotic fracture who had medication order but did not receive treatment offers a group of patients to target for intervention. For patients with neither medication order nor treatment, the provider or patient might be targeted. For future analysis, consider collecting standard documentation by physician of offer to prescribe osteoporosis therapy and patient response. This may offer additional patient subgroups to target for appropriate interventions that increase treatment rates in this vulnerable population.

Keywords: osteoporosis, fracture 
PS1-23:

Participation in Quality Improvement: Provider Perceptions Regarding Documentation Audits

\author{
Cheryl D. Pearce, ${ }^{1}$ Dana N. Rutledge ${ }^{1}$
}

\section{${ }^{1}$ California State University}

Background/Aims: Effective communication within health care depends on accurate documentation of assessments, plans and delivered care. Use of electronic medical records (EMRs) requires relearning and evaluating documentation processes for providers, and problems can arise when consistent use of entry fields is not maintained. At each phase of obstetric care, providers require accurate information. In shared practices, prenatal care consists of multiple office visits with multiple providers, focused on maternal/fetal status, accurate dating, and risk factor identification. Information accuracy is important at all phases, but especially so when laboring women present. In our HMO, all providers receive training on EMR use with further training following technology updates. Nevertheless, considerable variability exists in documentation practices. The EMR's comprehensive nature leads to different tabs, fields and pages where same information can be entered. Data entered into one tab/field may not automatically populate other fields as the provider expects (e.g. problem identified during visit does not make it to the list of patient problems entered on initial visit). Currently, there is no assessment of provider documentation skills or EMR completeness. This quality improvement project (QIP) aims for better documentation, with a secondary goal of mutual learning through peer auditing.

Methods: Our midwifery group, members of an obstetrical team in Southern California, initialized peer audits for initial prenatal care visits. Provider perceptions of reluctance, time involvement and learning (from the Conscious Competence Learning Model) are currently being evaluated before/after the initial cycle of peer audit process. All eligible midwifery service members are participating. The Fall 2014 QIP has two components: pre/post surveys and two rounds of EMR audits. Surveys will assess perceptions of time required for audits, attitudes about the effectiveness of audits and ideas for improving the audit process. Group meetings after each audit cycle will focus on concepts of shared learning and ideas for improving documentation.

Results: Comparison of audit composite objective ratings will detect changes in overall documentation behaviors. Survey results will determine whether the group's perceptions change and whether value is seen in ongoing audit cycles.

Discussion: This project will demonstrate whether using audit cycles enhance documentation and shared learning among participating midwives.

Keywords: peer documentation audits, perceptions of peer audits

PS1-26:

Application of Performance Improvement Methods to Improve Timeliness of Lung Cancer Care
Erin E. Hahn, ${ }^{1}$ Robert M. Cooper, ${ }^{1}$ Hugo Danilo, ${ }^{1}$ Alice H. Park, ${ }^{1}$ Elizabeth P. Bell, ${ }^{1}$ Iman A. Abdalla, ${ }^{1}$ Kien Vuu, ${ }^{1}$ Luis M. Moreta-Sainz, ${ }^{1}$ Michael K. Gould ${ }^{1}$

\section{${ }^{1}$ Kaiser Permanente Southern California}

Background/Aims: Timeliness, an essential element of high-quality care as defined by the Institute of Medicine, is critical in cancer care delivery. Timeliness of diagnosis and treatment is associated with improved outcomes for some cancers. A Kaiser Permanente Southern California (KPSC) organizational goal is to achieve optimal timeliness of cancer care to ensure best possible outcomes for KPSC members.

Methods: A working group at the KPSC Los Angeles Medical Center (LAMC), led by the physician director of the cancer program, the director of performance improvement and the director of oncology services, convened a series of multidisciplinary meetings focused on improving timeliness of lung cancer care. Participants included LAMC physicians, nurses and administrators as well as KPSC researchers. Alignment of physician education time and education credits was facilitated by administrative leaders. Performance improvement activities included review of current state of LAMC lung cancer care, definition of key metrics, iterative development of specialty-based process maps, chart abstraction and identification of potential interventions. Physicians participated in all activities, facilitated by group leaders. Data sources included medical records and tumor registry data.

Results: Review of LAMC baseline data for patients newly diagnosed with lung cancer in $2012(\mathrm{~N}=60)$ showed that the median number of days from initial imaging to date of diagnosis is 21 days (range: $0-112$ ). Median days from postdiagnosis physician consultation to initial treatment varied by physician specialty: 4 (radiology), 12 (radiation oncology), 24 (medical oncology) and 27 days (surgery). Chart review revealed that patients diagnosed in the inpatient setting had shorter time to receipt of services. Root cause analysis revealed several target areas for improvement interventions including: development of a lung cancer protocol for radiology, a closed-loop system with pulmonology, improved integration of palliative and hospice care, a rapid results system for genetic studies, and increased frequency of lung tumor board. A new position of lung cancer coordinator was proposed to oversee activities, and standardized "smart phrases" were created for documentation of encounters.

Discussion: Multidisciplinary groups with clinician, performance improvement and research participation can achieve significant progress in identifying areas for improvement using robust methods. Next steps will include implementation and evaluation of the proposed improvements.

Keywords: performance improvement, lung cancer timeliness

PS1-28:

Predicting, Recruiting and Tracking Enrollment of Young Adults to an Online Intervention to Support Healthy Eating 
Michelle Groesbeck, ${ }^{1}$ Michael Sheehan, ${ }^{1}$ Noel Maddy, ${ }^{1}$ Viji Ramaswami, ${ }^{2}$ Margaret Rukstalis, ${ }^{3}$ Ashley Ryman, ${ }^{3}$ Gwen L. Alexander ${ }^{1}$

\section{${ }^{1}$ Henry Ford Health System; ${ }^{2}$ University of Michigan; ${ }^{3}$ Geisinger Health System}

Background/Aims: Young adults, known as Generation Y, eat well below the daily recommended servings of fruits and vegetables and consume high rates of sweetened beverages. With a busy, ever-evolving lifestyle and lifelong exposure to the instant and browsable Internet, recruitment and engagement efforts require accommodating challenges of preference for quick food, quick read, and low attention to snail mail. We describe strategies and monitoring utilized to engage this diverse and understudied population in a twosite, online, randomized intervention trial (MENU GenY) designed to support healthy food choices.

Methods: Recruitment from two geographically diverse sites, metropolitan Detroit, MI, and rural Danville, PA, is ongoing. Automated data were utilized to identify young adult HMO members ages 21-30. Mailing strategy, envelope and invitation letter style were evaluated by focus groups across both sites and consultants in a two-phase process. Team meetings guided collaboration efforts on enrollment strategies, and technical meetings were established and run by project management to allow the programing staff across sites to focus and drive technology-specific developments on the study. Mailing volume, based on sample composition by predicted enrollment rates, was modeled across the 12-month recruitment timeframe. Tracking software was developed with two online applications, an internally built program at Henry Ford Health, Flash Manager V3, and an administrative console at University of Michigan CHCR, using the Django framework, to provide immediate feedback on enrollment rates overall and by site.

Results: An invitation letter was developed with a unique and catchy color study logo along with a color insert and magnet, sent in a business-style envelope, and followed by a second notice letter 10 days following. Young adults responded favorably to the second "reminder" letter, which nearly doubled enrollment rate, with the study on schedule to meet its $n=1,624$ goal. By comparing prediction to tracking, with consideration of enrollment by site and by gender, we adjusted mailing volume and rates to reach quarterly enrollment goals. Discussion: Through rigorous planning, collaborative team coordination and guidance by young adult advisors, we addressed challenges and experienced success with enrolling a hard-to-reach young adult population. We will provide enrollment updates and further details.

Keywords: recruitment, enrollment

\section{PS1-29:}

Choosing Wisely: Using the EHR to Identify Variability in Provider Ordering Behavior for HighEnd Imaging of the Head

Sharon Fuller, ${ }^{1}$ Roy Pardee, ${ }^{1}$ Eva Chang, ${ }^{1}$ Gabrielle Gundersen, ${ }^{1}$ Robert J. Reid, ${ }^{1}$ Matthew R. Handley, ${ }^{1}$ Diana S. Buist ${ }^{1}$

\section{${ }^{1}$ Group Health Cooperative}

Background/Aims: Over the last three years, a team of Group Health researchers and clinical leaders has worked collaboratively on projects to reduce low-value care. The team investigates, tests and implements metrics and interventions to improve care and reduce costs. The team's work is informed by the national Choosing Wisely campaign, which works with specialty medical societies to generate lists of low-value clinical activities. Group Health participates in the Washington State Choosing Wisely Task Force, which has identified and defined 10 high-priority recommendations on topics such as imaging, screening and antibiotic use.

Methods: Our team is developing methods to assess variability in clinician ordering behavior with regard to the Task Force recommendation to avoid high-end imaging for uncomplicated headache. Claims data, as in the Group Health virtual data warehouse (VDW), generally do not identify the ordering provider for radiology procedures, which typically have different ordering and performing providers. To understand ordering patterns, we are instead using clinical data to identify ordering provider, and to detect both completed and cancelled orders. We examine all electronic health record (EHR) orders with a CPT code denoting head computed tomography or magnetic resonance imaging, and identify orders with an ICD code signifying uncomplicated headache. All orders, whether completed or not, should be accompanied by a justifying diagnosis, although the specifics of radiology ordering add significant challenges to identifying these diagnoses. For completed orders, we gather additional diagnosis information from EHR billing data, which (unlike claims data) still identifies the ordering provider.

Results: We will compare the sensitivity of EHR ordering data, EHR billing data, and claims-based VDW data in identifying ordering of high-end imaging for headache. Achieving high specificity may not be feasible for this measure, given the resources required for chart review and programming exclusionary criteria. However, since our main goal at this point is to document variability between providers, high specificity for any individual provider is less critical.

Discussion: Once we validate measures for headache imaging, we will distribute data to clinics and providers for review and discussion, and develop measures for other imaging-related Task Force recommendations. We also will investigate the feasibility of adding ordering provider to Group Health VDW data for services provided in Group Health clinics.

Keywords: low-value care, Choosing Wisely

PS1-30:

Incidence of Venous Thromboembolism Following Major Surgery: Opportunities for Care Improvement

Michael K. Gould, ${ }^{1}$ Yi-Lin Wu, ${ }^{1}$ Amy Liu, ${ }^{1}$ Joel Handler, ${ }^{1}$ Helen Lau, ${ }^{1}$ Angelika Clarke,,${ }^{1}$ Kathy J. Lin, ${ }^{1}$ Tania Tang ${ }^{1}$

\section{${ }^{1}$ Kaiser Permanente Southern California}

Background/Aims: Venous thromboembolism (VTE) is an important cause of preventable death and morbidity 
following major surgery. However, few population-based studies have examined the perioperative incidence of VTE among surgical patients, and most studies limited followup to hospital discharge or 1 month following discharge. This study aimed to define the 90-day incidence of VTE in this population, examine trends in incidence over time and identify opportunities for improved use of pharmacological prophylaxis.

Methods: We performed a retrospective cohort study using data from electronic health records of adult patients admitted to one of 14 hospitals in an integrated health system between January 1, 2010, and March 30, 2013, for major nonorthopedic surgery, using relevant International Classification of Disease 9 (ICD-9) procedure codes from the Surgical Care Improvement Project of the Joint Commission. For this analysis, we defined VTE as the presence of a new ICD-9 diagnosis code for pulmonary embolism or deep venous thrombosis, when accompanied by either a pharmacy code for an anticoagulant medication at a dose appropriate for VTE treatment or an ICD-9 procedure code for an objective diagnostic test for VTE. In a prior validation study, this definition had positive and negative predictive values of almost $80 \%$. To capture incident VTE that occurred during hospitalization or within 90 days of discharge, we reviewed inpatient, outpatient, emergency department and claims files. Results: The sample included 27,723 surgical patients. Patients with VTE were substantially older, had more comorbid conditions and were more likely to be male, nonHispanic white, widowed and former smokers; $48 \%$ of all admissions were related to cancer. The crude risk of VTE was $1.25 \%$ over 90 days. The 90 -day incidence rate was 15.9 per 100,000 patient-days of follow-up (95\% confidence interval: 14.2-17.6). VTE incidence did not vary by calendar year, and relatively small differences were observed across hospitals.

Discussion: The incidence of VTE observed in this study compares favorably to that reported in several prior studies. Next steps include distinguishing preventable VTE from VTE that occurred despite receipt of prophylaxis, and development of risk assessment models to guide rationale use of either pharmacological or mechanical prophylaxis.

Keywords: venous thromboembolism, surgical outcomes

PS1-31:

\section{Perioperative Morbidity in Cirrhotic Patients Undergoing Orthopedic Surgery}

Eric M. Nyberg, ${ }^{1}$ Michael Batech, ${ }^{1}$ T. Craig Cheetham, ${ }^{1}$ Mary Alice Chocas, ${ }^{1}$ Anshuman Singh ${ }^{1}$

\section{${ }^{1}$ Kaiser Permanente Southern California}

Background/Aims: Previous studies have shown an increased risk of morbidity and mortality in cirrhotic patients undergoing surgery. However, there is a paucity of studies evaluating the perioperative risks of cirrhotics who undergo orthopedic surgery. The aim of this study was to examine outcomes in cirrhotic patients who have undergone orthopedic surgery.

Methods: This is a retrospective matched-cohort study using data from Kaiser Permanente Southern California. Study participants received a diagnosis for cirrhosis from $1 / 1 / 2003$ through 12/31/2013, and initial case selection criteria included age $>18$ years, $\geq 6$ months continuous health plan membership, and a procedure code for orthopedic surgery. At least 3 and up to 5 controls were optimally matched based on age, gender and cirrhosis diagnosis date. Data abstraction and summary were subsequently performed for demographic, socioeconomic, comorbid history and decompensation events data. Decompensation events included new onset ascites, variceal bleeding and hepatic encephalopathy. Multivariable conditional logistic regression estimated the risk of decompensation from surgery.

Results: We matched 4,263 eligible controls with 853 cirrhotic surgical patients. Mean age of the cohort was 60.5 (standard deviation: 11.44) years and 52.2\% were female. Within 90 days after surgery, cases had more decompensation events compared to matched controls (24.1\% vs. $19.1 \%)$.

Discussion: In this large database study including patients from a large managed care organization, cirrhotics experienced more decompensation within 90 days after orthopedic surgery compared to matched controls. Decompensation events result in significant increases in health care costs and utilization. Further, the increased morbidity seen in cirrhotics is a potential cause of quality issues for the managed care organization. Examination and mitigation of the factors, such as surgery, that can result in decompensation may help improve systems for better quality of care.

Keywords: cirrhosis, orthopedic postoperative outcomes

PS2-12:

Design and Implementation of a Clinician-Focused Intervention to Improve Diagnosis and Management of Symptomatic Vulvovaginal Atrophy in a Large Health System

Kimberly K. Vesco, ${ }^{1}$ Amanda Clark, ${ }^{1}$ Kate Beadle, ${ }^{1}$ Joanna Bulkley, ${ }^{1}$ Ashley Borin, ${ }^{1}$ Michael Leo ${ }^{1}$

\section{${ }^{1}$ Kaiser Permanente Northwest}

Background/Aims: Nearly 50\% of postmenopausal women experience symptoms related to vulvovaginal atrophy (VVA). However, despite the availability of effective treatment options, studies show that few women seek treatment for, and few providers ask women about, these symptoms. Our paired abstract describes clinician-reported barriers to diagnosis and management. Here we describe the prevalence of VVA diagnoses among women seen for well care within Kaiser Permanente Northwest (KPNW). We also describe the design of a clinician-focused intervention to improve diagnosis and management of symptomatic VVA.

Methods: Using electronic medical record (EMR) data, we identified well visits among women 55 years or older occurring within 17 KPNW primary care and OB/GYN clinics between June 2013 and May 2014. We computed the proportion of women who received a VVA-related diagnosis at the index visit. We then stratified the clinics based on visit volume and randomized them to an immediate versus delayed clinician- 
focused intervention. The intervention includes online and in-person education for clinicians regarding: 1) diagnosis and management of symptomatic VVA, and 2) new EMR-based clinical support tools (Smartsets and Smarttexts).

Results: We identified 14,274 unique well visits over the 1 -year period of time. Of these, $80.5 \%(11,500 / 14,274)$ occurred in primary care. Only $5.7 \%(769 / 13,544)$ of visits by women not already using vaginal estrogen contained a VVArelated diagnosis. There are 371 clinicians; education for those in the intervention clinics is ongoing (Fall 2014). After the education period, we'll prospectively collect EMR data to compare groups on the following outcomes: 1) proportion of well visits with VVA diagnoses; 2) proportion of women receiving vaginal estrogen prescriptions; and 3) proportion of visits that used the clinical support tools. We will also conduct an online survey of patients to determine if women in the intervention clinics are more likely than those in the control clinics to discuss VVA symptoms with their providers and to receive education and treatment options if symptomatic.

Discussion: The proportion of postmenopausal women with a VVA-related diagnosis was lower than expected. The purpose of our ongoing study is to evaluate whether active outreach to providers with education and clinical support tools leads to improved diagnosis and management of VVA.

Keywords: patient care improvement, postmenopause

\section{PS2-13:}

\section{Ready to Quit: Predictive Analysis on Member Readiness of Enrolling Smoking Cessation Programs in Kaiser Permanente Southern California}

\section{Di Meng, ${ }^{1}$ Terhilda Garrido ${ }^{1}$ \\ ${ }^{1}$ Kaiser Permanente Program Office}

Background/Aims: We describe a predictive analysis to help regional operations engage smoking members with high likelihood of enrollment into smoking cessation services.

Methods: A combination of investigation methods was used: 1) data mining and basic free text parsing and recognition; 2) predictive modeling to discover predictors of member enrollment in smoking cessation services and score the future population to identify smoking members with high likelihood of enrollment; and 3) randomized control trial to (a) identify the best protocols to outreach these members, and (b) refine the model and establish a learning cycle to guide operations.

Results: We have identified and processed a wide range of predictors including smoking habit, social/economic factors, enrollment history, significant medical conditions, family characteristics, medical utilization history, online interaction and other health/social behaviors. The model performed very well in validation process, with c-stat of 0.76 , which provides a good fit and predicts power. The findings of significant predictors were in agreement with many literatures. These predictors include past enrollment, sex and disease burden. We also found that race/ethnicity and online activity, especially patient/physician secure email communication on smoking matter, strongly predict likelihood of enrollment.

Discussion: In the Kaiser Permanente Southern California region, approximately 200,000 smokers can be identified through social history and ICD-9 codes in Kaiser Permanente HealthConnect. The top $20 \%$ of the modeled population represents $50 \%$ of potential enrollees in smoking cessation programs, and this population will be targeted in the outreach effort in a randomized control trial designed as follows. A population of 10,000 will be randomly selected to not participate in phase one, but will be eligible to participate after the first phase. Of this 10,000, 5,000 will receive no intervention and 5,000 will receive only existing auto-dialer outreach, both of which are within the normal standard of care. The remaining 30,000 members ( 10,000 per month) will be randomized by medical center into three treatment groups of equal size: auto-dialer plus live phone call, auto-dialer plus letter, and auto-dialer plus email.

Keywords: predictive modeling, smoking cessation

PS2-18:

Improving Engagement in Addiction Treatment: Translating Addiction Research Into EvidenceBased Practice

Rulin C. Hechter, ${ }^{1}$ Scott B. Sangsland, ${ }^{1}$ Grant R. Grissom, ${ }^{2}$ Jim R. Carter, ${ }^{3}$ Catherine F. Jervey, ${ }^{3}$ Sean E. Koon, ${ }^{3}$ Ashley Hooker ${ }^{2}$

${ }^{1}$ Kaiser Permanente Southern California; ${ }^{2}$ Polaris Health Directions; ${ }^{3}$ Southern California Permanente Medical Group

Background/Aims: Patient engagement is critical for the success of addiction treatment. We sought to evaluate the effectiveness of an innovative patient engagement enhancement system, Enhanced Engagement in Chemical Dependency (EECD), for improving patient engagement in addiction treatment.

Methods: The EECD system promotes motivational interviewing/motivational enhancement therapy (MI/MET) and service matching approaches, an evidence-based practice that can improve engagement. It includes two components: 1) staff training in MI/MET, and 2) a standardized webbased engagement assessment with real-time clinical and personalized patient reports and program-level management reports. We will roll out a staggered implementation in 12 outpatient addiction treatment programs at Kaiser Permanente Southern California, where about 8,000 new intakes were conducted per year. The analysis employs a stepped wedge design, in which all adults who have completed an intake in the 6 months prior to implementation will serve as internal baseline controls, while all adults who have completed an intake in the sites that have not yet implemented the system will serve as external controls for the implementation sites. We will evaluate the effectiveness of the EECD system on: 1) improving treatment engagement (attendance at two sessions within 30 days after the initial treatment visit); 2) authentic participation in outpatient treatment; 3) clinical outcomes (addiction severity index); 4) reducing treatment reentry within 6 months following the end of a treatment episode; 5) matching services to patient needs; and 6) sustained effectiveness in 
improving engagement. Generalized estimating equations will be used to test for significant differences in proportion of patients with favorable outcomes on binary variables. Linear mixed models will be used for continuous outcome variables. Results: Staff training and a staggered implementation of the EECD system will be rolled out in 12 sites during October 2014-April 2015, with two initial sites in phase I, five sites in phase II and five sites in Phase III. The results are forthcoming. Discussion: This systemic intervention project represents an important example of translating research into improving patient care in addiction treatment, through a multidisciplinary collaboration between researchers, providers and operational leaders. The findings from this project will facilitate adoption of evidence-based practices and innovative technologies in addiction treatment.

Keywords: addiction treatment, patient engagement

\section{PS2-20:}

Technical and Data Collection Challenges and Lessons Learned in the Pain Program for Active Coping and Training (PPACT) Pragmatic Trial

\section{Reesa L. Laws, ${ }^{1}$ Alee Rowley ${ }^{1}$ \\ ${ }^{1}$ Kaiser Permanente Northwest}

Background/Aims: Chronic pain affects at least 116 million adults in the United States and exacts a tremendous cost in suffering and lost productivity. While health systems offer specialized pain services, the primary care setting is where most patients seek and receive care for pain. The Pain Program for Active Coping and Training (PPACT) project is testing the effectiveness of integrating an evidence-based, interdisciplinary pain management intervention within a primary care environment.

Methods: The Kaiser Permanente Northwest (KPNW), Georgia (KPGA) and Hawaii (KPHI) regions are working with their regional health partners to recruit and implement the PPACT trial. Efforts at each clinical site involve extracting electronic medical record data via the virtual data warehouse and Clarity. KPNW built a tracking system to manage all the recruitment and implementation processes across the three regions. The tracking system serves to: 1) house records of study eligible patients; 2) manage touches with the PCPs prior to recruitment to ensure we recruit patients whom PCPs specifically believe this program would work best; 3) keep track of the recruitment efforts; 4) track all intervention activities; and 5) aide in data collection processes using KP.org questionnaires and Kaiser Permanente Message Center IVR surveys.

Results: We have implemented recruitment efforts across all regions using a staggered cohort approach. KPNW started cohort 1 as a pilot to firm up all tracking, recruitment and intervention processes. Both KPGA and KPHI are now in the field as well. We are continuing to refine our processes to meet the recruitment challenges we have encountered in the regions.

Discussion: Running a pragmatic trial embedded within a health care system is challenging but possible. Adequate collaboration with regional leaders, clinical managers and PCPs to learn the needs of patients and meet expectations for a complex trial is crucial, as is sufficient time to set up data collection processes within the health care setting and test research tracking systems and processes necessary to make operations run as smooth as possible. This presentation will highlight the technical and data collection challenges and lessons learned for conducting a pragmatic trial in a health care setting.

Keywords: pragmatic, data collection

\section{CHRONIC CONDITIONS/DIABETES/ OBESITYIMULTIPLE CHRONIC CONDITIONS}

\section{A3-1: \\ Impact of Comorbidity Dyads on Heart Failure Treatment in Older Persons}

Jerry H. Gurwitz, ${ }^{1}$ Alan S. Go, ${ }^{2}$ Sue Hee Sung, ${ }^{2}$ Grace Tabada, ${ }^{2}$ Robert Goldberg, ${ }^{3}$ David J. Magid, ${ }^{4}$ David H. Smith, ${ }^{5}$ David McManus, ${ }^{3}$ Jane S. Saczynski, ${ }^{1}$ Bruce Barton $^{3}$

${ }^{1}$ Meyers Primary Care Institute; ${ }^{2}$ Kaiser Permanente Northern California; ' ${ }^{2}$ niversity of Massachusetts; ${ }^{4}$ Kaiser Permanente Colorado; ${ }^{5}$ Kaiser Permanente Northwest

Background/Aims: The vast majority of heart failure (HF) patients suffer from multiple noncardiac comorbid conditions that may have important implications for clinical management. We assessed the clinical effectiveness and safety associated with the use of beta-blocker therapy for patients in a HFchronic lung disease dyad group, and the clinical effectiveness and safety associated with the use of angiotensin-converting enzyme (ACE) inhibitors/angiotensin II receptor blockers (ARBs) for patients in a HF-chronic kidney disease (CKD) dyad group.

Methods: We identified over 30,000 HF patients with a documented ejection fraction (EF) from four members of the Cardiovascular Research Network - Kaiser Permanente Northern California, Colorado and Northwest, and Fallon Health-Meyers Primary Care Institute - from January 1, 2005, through December 31, 2008. To be included in the cohort, patients had to have $>1$ inpatient admission with primary diagnosis of $\mathrm{HF}$ and/or $>3$ outpatient encounters with any diagnosis of HF. Outcomes of interest included: death, hospitalization due to heart failure, and hospitalization for any cause.

Results: Beta-blocker therapy was associated with a reduced risk for death among the overall group of HF-lung disease patients, and specifically for those with borderline or reduced EF. ACE/ARB therapy was associated with a reduced risk for death among the overall group of HF-CKD patients, and specifically for those with preserved and reduced EF. We did not detect a statistically significant association between beta-blocker use and hospitalization for HF or hospitalization for any cause among the overall group of HF-lung disease 
patients; however, there was a reduced risk observed in hospitalization from any cause for those with reduced EF. We did not detect a statistically significant association between $\mathrm{ACE} / \mathrm{ARB}$ use and hospitalization for HF or hospitalization for any cause among the overall group of HF-CKD patients; however, there was a reduced risk observed in hospitalization for HF among those with borderline EF.

Discussion: The findings of this observational study may have clinical implications for the management of $\mathrm{HF}$ in patients with common comorbid conditions including chronic lung disease and chronic kidney disease.

Keywords: aging, multiple chronic conditions

\section{A3-2: \\ Do Patients With Multiple Chronic Conditions Experience the Same Care as Patients With Fewer Chronic IIInesses? Insights From Videos of Primary Care Office Visits}

\section{Ming Tai-Seale, ${ }^{1}$ Weimin Zhang ${ }^{2}$ \\ ${ }^{1}$ Palo Alto Medical Foundation Research Institute; ${ }^{2}$ Samueli Institute}

Background/Aims: How do primary care physicians (PCPs) manage multiple chronic conditions (MCC) patients given the financial incentives in their practices?

Methods: Qualitative and quantitative analyses of a convenience sample of 385 video recordings of older patientPCP conversations in three primary care practice settings - an academic medical center (AMC) with salaried PCPs, a fee-forservice (FFS) managed care group (MCG) and several FFS innercity solo practices (ICS) — in the Midwest and Southwest. We identified topics - issues requiring a response from either patient or physician - that emerged during a visit and categorized them according to a coding system. We recorded the time spent on each topic and visit length. To measure the topic's severity, we mapped the topics to the five top leading causes of disabilities (LCD) identified by the National Center for Health Statistics: pain, cardiovascular diseases, diabetes, lung diseases and mental illnesses. We assessed patient-centeredness by physician's empathy, warmth and engagement in informed decision-making. Generalized estimation equation (GEE) analyses examined the pattern of time use and patient-centeredness across patients with 0 LCD, 1 LCD or $\geq 2$ LCDs (i.e. MCC).

Results: Over 56\% (216/385) of visits contained $\geq 2$ LCDs. Average length of visits with 0 LCD lasted 13.21 minutes, whereas visits with $1 \mathrm{LCD}$ lasted 16.63 minutes, and averaged 18.93 minutes for $\geq 2$ LCDs. MCC visits in the AMC were longer (23.91 minutes), followed by MCG (14.82 minutes) and ICS (12.42 minutes). Within visits, GEE findings suggested if a visit had only 1 LCD, time spent on the LCD was 2.90 minutes. If a visit had $\geq 2$ LCDs, time on each LCD was reduced to 2.51 minutes. Compared to FFS PCPs, AMC physicians not only spent more time (2.95 vs. 2.23 minutes/ LCD) with MCC patients, their communication also was more patient-centered. Qualitative analyses of MCC visits revealed a sometimes narrow focus on some individual LCDs while overlooking others.
Discussion: MCC patients experienced longer visits, although the increase in number of LCDs is associated with a reduction in the time spent on each LCD within the visit. Salaried AMC physicians not only spent more time with MCC patients, but also were more patient-centered in communication. Payment policies that reward value over volume could facilitate patientcentered care for MCC patients.

Keywords: aging, multiple chronic conditions

A3-3:

Higher Prevalence of Insulin Resistance Among Asian Americans Despite Lower Body Mass Index

Jia Pu, ${ }^{1}$ Robert Romanelli, ${ }^{1}$ Beinan Zhao, ${ }^{1}$ Sukyung Chung, ${ }^{1}$ Vani Nimbal, ${ }^{1}$ Latha Palaniappan ${ }^{1}$

\section{${ }^{1}$ Palo Alto Medical Foundation Research Institute}

Background/Aims: This study aims to identify racial/ ethnic differences in the relationship between body mass index (BMI) and insulin resistance across Asian American subgroups (Asian Indian, Chinese, Filipino, Japanese, Korean, Vietnamese), Mexicans, non-Hispanic blacks (NHBs) and non-Hispanic whites (NHWs) in a large, mixedpayer ambulatory care setting in northern California.

Methods: This cross-sectional study examined electronic health records of patients aged 18 years or older from 20002012. Insulin resistance was indicated by triglyceride/highdensity lipoprotein ratio $>3$. Overweight or obesity was defined as $\mathrm{BMI} \geq 25$.

Results: Although Asian Americans had the lowest rate of overweight and obesity, they had the highest prevalence of insulin resistance in both normal weight $(19 \%, 9,262$ of $49,981)$ and overweight/obese groups (43\%, 15,742 of 36,496), followed by Mexicans (normal: 12\%, 197 of 1,590; overweight: $39 \%, 2,106$ of 5,377), NHWs (normal: 9\%, 6,271 of 65,921; overweight: $32 \%, 33,475$ of 103,695 ) and NHBs (normal: $6 \%$, 46 of 716 ; overweight: $16 \%, 459$ of 2,855). After controlling for age, gender and important comorbidities (presence of high blood pressure, hyperglycemia, dyslipidemia), Asian Americans were still significantly more likely to have insulin resistance in both BMI groups (normal: 1.6 odds ratio [OR], 1.5-1.7 confidence interval [CI]; overweight: 1.3 OR, 1.2-1.4 CI) compared to NHWs. Significant racial/ethnic differences in insulin resistance were also found across Asian American subgroups. Among normal weight patients, Asian Indians (OR: 2.1, CI: 2.0-2.2), Chinese (OR: 1.4, CI: 1.3-1.5) and Filipinos (OR: 1.3, CI: 1.1-1.5) were significantly more likely to have insulin resistance compared to NHWs after controlling for age, gender and comorbidities. Higher adjusted risk of insulin resistance was also observed among Asian Indians (OR: 1.5, CI: 1.4-1.6) and Chinese (OR: 1.3, CI: 1.2-1.4) overweight/ obese patients, while other Asian subgroups had no significant difference compared to NHWs.

Discussion: Asian Americans are more likely to have insulin resistance despite lower rates of overweight and obesity. Our study suggests the use of lower BMI when screening for insulin resistance among Asian Americans.

Keywords: insulin resistance, racial/ethnic differences 
A3-4:

\section{Bisphosphonate Drug Holiday and Fracture Risk}

Annette L. Adams, ${ }^{1}$ John L. Adams, ${ }^{1}$ Marsha A. Raebel, ${ }^{2}$ Beth T. Tang, ${ }^{1}$ Jennifer L. Kuntz, ${ }^{3}$ Vinutha Vijayadeva, ${ }^{4}$ Elizabeth A. McGlynn, ${ }^{1}$ Wendolyn S. Gozansky ${ }^{2}$

${ }^{1}$ Kaiser Permanente Southern California; ${ }^{2}$ Kaiser Permanente Colorado; ${ }^{3}$ Kaiser Permanente Northwest; ${ }^{4}$ Kaiser Permanente Hawaii

Background/Aims: Among women with $\geq 3$ years exposure to bisphosphonates (BPs), we compared the incidence of fragility fractures in those who discontinued BPs for $\geq 12$ months (drug holiday) to those who continued to use BPs (persistent use).

Methods: This retrospective cohort study included women aged $\geq 45$ years who initiated BP use from four Kaiser Permanente regions between January 1, 1998, and December 31, 2009. Drug holiday was defined as $\geq 12$ months with BP use at $0 \%$ adherence. Persistent use status required ongoing use at $\geq 50 \%$ adherence. The primary outcome of interest was the first occurrence of an incident clinical osteoporosis-related fragility fracture, identified from the electronic medical record (EMR) via ICD-9$\mathrm{CM}$ codes. All subjects were followed until fracture, disenrollment from the health plan, death, or December 31, 2012. From the EMR, we collected information on the following potential confounders and effect modifiers: race/ ethnicity, age, body mass index, comorbidities, history of previous fragility fracture, lowest $\mathrm{T}$-score prior to cohort entry, fall risk, 10-year fracture risk, and prior/concomitant use of bone-active medications. Persistent users and drug holiday subjects were compared with regard to several demographic and clinical characteristics. Time-varying Cox proportional hazards models were used to compare osteoporosis-related fracture incidence between the two groups.

Results: The cohort of 28,620 women, observed for 111,997 person-years, included 17,123 (59.8\%) persistent BP users and $11,497(40.2 \%)$ drug holiday subjects. The drug holiday group had fewer comorbidities, higher baseline T-scores, and lower fracture and fall risk scores. A total of 3,571 osteoporosis-related fractures were observed. The unadjusted rate ratio (RR) for any osteoporosis-related fractures for drug holiday compared to persistent use was 0.87 (95\% confidence interval [CI]: $0.81-0.94)$, but was 1.0 (95\% CI: $0.9-1.2$ ) for hip fractures only. The time-varying models suggested no differences in fracture risk (hazard ratio [HR]: 0.90, 95\% CI: $0.80-1.00$ ) after adjustment for baseline fall and fracture risk, comorbidities and other bone-active medication use. Similarly, no difference in hip fracture risk was observed (HR: 0.84, 95\% CI: 0.68-1.03).

Discussion: Women who undertake a holiday from BP use are not at greater risk of osteoporosis-related fragility fractures, nor hip fractures specifically, than are women who continue to use BPs persistently.

Keywords: osteoporosis, fracture risk
B4-2:

Improving Lifestyle Interventions for People With Serious Mental Illnesses: Qualitative Results from the STRIDE Study

Scott Stumbo, ${ }^{1}$ Bobbi Jo H. Yarborough, ${ }^{1}$ Micah T. Yarborough, ${ }^{1}$ Thomas J. Young, ${ }^{1}$ Carla A. Green ${ }^{1}$

\section{${ }^{1}$ Kaiser Permanente Northwest}

Background/Aims: Overweight and obesity are disproportionately prevalent among individuals with serious mental illnesses, in part due to psychiatric medications, poor dietary habits and sedentary lifestyles. Behavioral lifestylechange interventions are effective yet require more than training participants on energy balance. Interventions must address factors relevant to the context within which people attempt to lose weight and improve health, such as lack of the following: nutrition education, access to and affordability of healthy foods, safe places to exercise and skills.

Methods: As part of a randomized controlled trial $(\mathrm{N}=200)$ that was successful in producing significant weight loss at 12 months, we conducted 101 semistructured interviews with 84 participants to understand barriers and facilitators of weight loss and lifestyle changes associated with the intervention, or with efforts at losing weight and remaining physically active more generally. Interviews were coded by a team (with $79 \%$ agreement among coders) and were analyzed using Atals.ti.

Results: Interviewees averaged 48 years old; $36 \%$ were men, $21 \%$ were nonwhite, and $24 \%$ were from the control group. Participants had diagnoses of schizophrenia or schizoaffective disorder (41\%), bipolar disorder (20\%), affective psychosis $(37 \%)$ or posttraumatic stress disorder (2\%). Thematic analyses revealed a number of key facilitators and barriers. Facilitators included: 1) current health concerns that precipitated the decision to engage in a lifestyle intervention; 2) group camaraderie facilitating participation and weight loss during the intervention; and 3) social support for exercise. Barriers included: 1) loss of structured support and subsequent difficulties continuing with lifestyle improvements postintervention; 2) bad weather as a deterrent to exercise; 3 ) the interaction of mental health symptoms (e.g. depression, anxiety) with disinhibited eating; and 4) all-ornothing thinking that led to defeatist attitudes about weight loss.

Discussion: Successful weight loss can be achieved among individuals with serious mental illnesses with the right intervention. We found a strong preference for consistent, group-based support to foster a sense of accountability, which motivated behavior changes. Lifestyle change interventions for this population should also help participants develop flexible cognitive restraint and the ability to iteratively cope and adapt to changes in mood and subsequent changes in motivation to eat healthfully and exercise regularly.

Keywords: serious mental illnesses, behavioral change

D3-3:

Do Financial Incentives Encourage Health Plan Members to Complete Health Risk Assessments? 
Jason P. Block, ${ }^{1}$ Dennis Ross-Degnan, ${ }^{1}$ Matthew W. Gillman, ${ }^{1}$ Sheryl L. Rifas-Shiman, ${ }^{1}$ Renata Smith, ${ }^{1}$ Ken Kleinman $^{1}$

\section{${ }^{1}$ Harvard Pilgrim Health Care}

Background/Aims: Employers increasingly offer financial incentives to employees for participation in wellness activities. Whether these incentives are effective is unclear. In a nonprofit health plan, we examined the impact of monetary incentives on completion of a health risk assessment (HRA).

Methods: Harvard Pilgrim Health Care, a nonprofit HMO in New England, began encouraging members to complete a HRA starting in 2010. Any health plan member was eligible to complete a HRA, which included questions about health behaviors, quality of life and medical conditions. Starting in 2011, some large employers who contract with Harvard Pilgrim Health Care for employee health insurance began incentivizing completion of the HRA. Using records on health plan members, the HRA and employer incentives, we gathered data on all adult health plan members from October 2010 to July 2013 ( $\mathrm{N}=991,743)$. For this analysis, we compared completion of a HRA among the 66,639 members who received incentives versus the 925,104 members who did not, adjusted for demographics. We used survival analysis to examine the association of receiving a HRA incentive with HRA completion.

Results: Among members, mean age was 42.6 years, $52 \%$ were female, and $89 \%$ were white. During the 2.75 -year followup period, 18,884 members completed a HRA, including 11,254 among the 66,639 with incentives $(16.9 \%)$ and 7,630 among the 925,104 without $(0.8 \%)$. In multivariable models controlling for demographics, being offered an incentive was strongly associated with time until completion of a HRA in 2011, 2012 and 2013 (hazard ratios: 6.0 [95\% confidence interval: 5.6-6.4] for 2011, 29.2 [28.0-30.5] for 2012 and 46.4 [41.1-48.8] for 2013). Other important predictors of HRA completion included female gender, Hispanic or "other" race (vs. white race), and older age. Black members were less likely to complete the HRA than white members. Larger incentives were more successful in promoting HRA completion than smaller incentives.

Discussion: Providing financial incentives was strongly associated with completion of a health risk assessment in a nonprofit health plan.

Keywords: financial incentives, health risk assessment

PS1-17:

Should Pharmacists Be Included in a Core Team to Reduce Hospital Admissions and Mortality for Patients With Chronic Heart Failure?

Indubala Vardhan, ${ }^{1}$ Yvonne E. Grant, ${ }^{1}$ Carmela Astrea, ${ }^{1}$ Joanna Wolmer, ${ }^{1}$ Paula Hinz ${ }^{1}$

${ }^{1}$ Kaiser Permanente Southern California

Background/Aims: Heart failure (HF) is a common disease and accounts for multiple hospital admissions, readmissions, increasing health care costs and high mortality. One in five Americans is diagnosed with HF. Symptoms are mostly managed with medications. Evidence shows that a multidisciplinary care team is best practice for managing HF. More research is needed to determine ideal combinations for services on a core team to optimize use of resources and intervention effectiveness. We aimed to determine the impact on outcomes of adding a clinical pharmacist intervention to a HF multidisciplinary care team. We hypothesized that a pharmacist would increase efficiency of the team, and thereby improve outcomes of decreased hospitalization and mortality. Methods: We conducted a prospective, pretest-posttest nonrandomized, single-arm, pilot study to determine impact of adding a pharmacist intervention. In an ambulatory care setting, 100 patients who were hospitalized with HF in prior 12 months were invited to participate; 81 patients consented, enrolled and completed the study. Retrospective data on 81 other comparable patients, admitted for HF in prior 12 months, served as controls. Control patients had received usual care from the multidisciplinary care team, whereas study patients received usual care plus pharmacist intervention. The intervention R-E-A-D was comprised of four steps - Step 1: Reconciliation of medications; Step 2: Education to patient/ caregiver on indications for medications; Step 3: Adherence evaluation; and Step 4: Drug monitor for drug adverse effects, drug/disease interactions and therapy optimizations. The study was conducted from May 2010 to November 2011.

Results: Demographic variables included average age 75 years, average medications 10 and average comorbidities 5 . At 12 months postintervention, the control group realized a decrease of 32 hospital readmissions and a reduction of 60 hospital days $(\mathrm{P}=0.16)$, while the intervention group realized a decrease of 97 readmissions and a reduction of 332 hospital days $(\mathrm{P}<0.001)$. Postintervention, 23 of 81 patients $(28 \%)$ had expired in the control group, while 13 of 81 patients (16\%) had expired in the treatment group.

Discussion: The study confirms that pharmacists should be included as members of a core team to reduce readmissions and mortality for patients with chronic HF.

Keywords: heart failure intervention, chronic disease management

\section{PS1-27: \\ Medical Assistants as Health Coaches: Helping Obese Patients Set Goals}

Paula Lozano, ${ }^{1}$ June BlueSpruce, ${ }^{1}$ Dori Rosenberg, ${ }^{1}$ Kim Wicklund, ${ }^{1}$ Karen Severson ${ }^{1}$

\section{${ }^{1}$ Group Health Cooperative}

Background/Aims: Obesity is a common condition seen in primary care. However, there have been few programs for obese patients within existing primary care infrastructure. We sought to develop a program to train medical assistants to provide brief health coaching on weight management and help patients set goals.

Methods: Coaching was based on motivational interviewing and brief action planning. We used a mixed-methods, iterative 
approach to refine the intervention to prepare for broader dissemination. We summarize key events, themes and data collected.

Results: We convened a team of researchers, providers and operational leaders. In the first 2 months, we trained all 8 medical assistants (MAs) in one primary care clinic and worked with them to launch the intervention. A senior interventionist mentored and provided supervision to the MAs over several improvement cycles during a 4-month period. We conducted qualitative interviews to obtain feedback 6 months into the project. Barriers included patient reluctance to talk about weight management, preference for more provider involvement, and time constraints. Facilitators included feeling that the topic is important, support from the supervision and training team, and positive coaching experiences. We made changes to the intervention based on the interviews and meetings with the clinic providers. During a 1 -month period 8 months after launch, there were 1,143 adult primary care encounters to an average of 5 primary care providers over 21 clinic days. One-third $(36 \%, n=409)$ were by patients with a body mass index $>30$, and of these, $5.4 \%$ of patients were coached by an MA. The MAs coached a total of 23 patients, or 5.5 patients per week.

Discussion: Brief health coaching by MAs coaching may be feasible once key barriers are addressed. We are currently piloting revised procedures and continuing to mentor MAs and providers through 2014.

Keywords: health coaching, behavior change

PS2-1:

Defining Care Needs in Older Adults With a Particular Set of Multiple Chronic Conditions: Coexisting Visual, Auditory and Cognitive Impairments

Heather E. Whitson, ${ }^{1}$ Phillip Liu, ${ }^{2}$ Gerda G. Fillenbaum, ${ }^{3}$ Bruce M. Burchett, ${ }^{3}$ Harvey J. Cohen ${ }^{3}$

${ }^{1}$ Duke University Health System; ${ }^{2}$ Duke-National University of Singapore; ${ }^{3}$ Duke University

Background/Aims: Patients who have multiple chronic conditions (MCCs) represent a majority of older adults and have high health care needs. Considering the heterogeneity of MCC, one approach to identifying specific need gaps is to focus on common pairs or clusters of MCCs. Visual, auditory and cognitive impairments are common in old age, frequently coexist, and impact function. However, little is known about their combined association with patient-centered outcomes in older adults. Our objective was to assess the relationship of disability and self-reported health with various combinations of coexisting visual, auditory and/or cognitive impairments.

Methods: We analyzed sample data from the North Carolina EPESE study ( $\mathrm{N}=3,871$; age 65-105 years), a populationbased epidemiological study of community-dwelling seniors. Cognition was assessed by the Short Portable Mental Status Questionnaire, and visual and auditory status determined from self-reported information. Logistic regression characterized the cross-sectional associations of all combinations of visual, auditory and cognitive impairment with disability in activities of daily living (ADL), instrumental ADL (IADL) and poor self-reported health (SRH).

Results: The prevalence of ADL disability was 9.9\% (384 of $3,871)$, IADL disability $29.6 \%$ (1,146 of 3,871$)$, and fair/poor SRH $46.6 \%$ (1,805 of 3,871). In adjusted analyses, compared to persons with no impairments, persons with all three impairments had increased odds of ADL disability (odds ratio [OR]: 4.99, 95\% confidence interval [CI]: 2.95-8.44), IADL disability (OR: 10.39, 95\% CI: 5.67-19.05) and low SRH (OR: 2.81, 95\% CI: 1.64-4.82). The relationship between impairments and ADL disability exhibited a "step-wise" pattern (odds of disability increased as number of impairments increased), but IADL disability was particularly common in those with vision impairment, cognitive impairment, or the combination. While sensory impairments were associated with poor SRH, cognitive impairment was not unless both sensory impairments were present.

Discussion: Older adults with coexistent sensory and cognitive impairments have high functional needs. People with combined vision and hearing loss are likely to perceive their health as poor, whereas cognitive impairment may confer some protection against illness perceptions. Persons with coexisting visual and cognitive impairments are especially likely to require assistance with IADLs (e.g. medication management, handling finances) and may benefit from targeted clinical services that help overcome those impairments.

Keywords: aging, multiple chronic conditions

\section{PS2-2:}

\section{Factors Associated With Time to Diagnosis in Fibromyalgia}

Gabriel Chodick, ${ }^{1}$ Daliah Weitzman, ${ }^{1}$ Yael Bar-On, ${ }^{2}$ Varda Shalev, ${ }^{1}$ Howard Amital ${ }^{3}$

\section{${ }^{1}$ Maccabi Healthcare Services; ${ }^{2} T e l$ Aviv Medical Center; ${ }^{3}$ Sheba Medical Center, Tel HaShomer}

Background/Aims: The diagnosis of fibromyalgia, a chronic debilitating disorder, is complicated and affected by various factors of the patient and the caregiver. The objectives of this study were to investigate the time passing from initial complaints to diagnosis and to delineate the patient and physician characteristics affecting that time.

Methods: For this retrospective cohort study we used the datasets of Maccabi Health Services (MHS), the second largest health maintenance organization in Israel. We identified all confirmed cases of fibromyalgia that were diagnosed by specialists in the community or hospital during $1 / 2008-12 / 2011$. These patients were compared to sex- andage-matched fibromyalgia-free members. Different complaint patterns were examined to ascertain time of initial complaints. The pattern with the best validity was applied on nonvalidated cases that were diagnosed with fibromyalgia by primary physician, rheumatologist or at release from hospitalization during the same period. Patient and primary physician factors associated with time between initial complaints and fibromyalgia diagnosis were assessed. A multilevel generalized mixed linear model with a log-linked gamma 
distribution was used to account for clustering of patients associated with the same primary physician.

Results: Our study included 2,656 confirmed cases of fibromyalgia (91\% women, mean age: 51.1 years, standard deviation [SD]: 11.3 years). The most valid initial complaints pattern included 4 or more complaints within 6 months, found in $73 \%$ of the cases. These results indicated that the mean duration between initial fibromyalgia-related complaints to final diagnosis was 4.7 years with SD of 3.6 years. In a multivariable model, shorter time to diagnosis was associated with patients of young age, male gender and higher socioeconomic status, and with primary physicians of young age, family/pediatric/internal specialty versus general, and medical studies in Eastern or Western Europe versus other continents. Discussion: The mean time to diagnosis of fibromyalgia is very long and is significantly influenced by patient and physician characteristics. Better patient and physician education, and increased awareness to the disease and its early complaints, can be a key for improving the diagnosis of fibromyalgia.

Keywords: fibromyalgia, health care services

\section{PS2-3:}

\section{The Need for New Care Strategies to Prevent A1c Relapse}

JoAnn M. Sperl-Hillen, ${ }^{1}$ Heidi L. Ekstrom, ${ }^{1}$ Patrick J. O'Connor, ${ }^{1}$ Richard M. Bergenstal, ${ }^{2}$ Stephen E. Asche, ${ }^{1}$ Terese A. DeFor, ${ }^{1}$ Gerald H. Amundson, ${ }^{1}$ Deepika Appana $^{1}$

\section{${ }^{1}$ HealthPartners; ${ }^{2}$ Park Nicollet}

Background/Aims: The principle treatment strategy for glycemic management in most care settings is reactive; monitor A1c levels and then react with treatment intensification when the A1c exceeds the recommended optimal care goal. Our goal was to assess the potential to improve diabetes performance measures through preventive strategies directed at patients who are at A1c goal but at high risk for disease progression and A1c relapse.

Methods: Patients not meeting optimal care goals were partitioned into one of three different A1c trajectories: (a) FLAT - those who are consistently above optimal A1c goal, (b) Negative slope - those patients who are on an improvement trajectory, and (c) Positive slope - those who have previously been meeting A1c goals but who have relapsed (often due to medical issues, comorbidities, psychosocial stress, behavioral or medication adherence, or disease progression). We quantified the proportion of patients with diabetes who contribute to the relapse vector by identifying patients with diabetes and A1c tests in the last two years (9/1/2012-8/31/2014) and quantifying the proportion of patients who relapsed in year 2 , stratified by A1c range and pharmacologic treatment in year 1 .

Results: We identified 29,321 patients with at least two diabetes diagnoses in years 1 and 2, with median A1c of $7.4 \%$. Of these, 8,889 (30\%) had an A1c > 8\% in year 2. Of 6,321 patients with A1c of 7-7.9\% in year 1, 2,332 (36.9\%) relapsed to $>8 \%$ in year 2 . Relapse was higher $(43.2 \%)$ for patients medicated with sulfonylurea or insulin. Only $689 / 10,202$ $(6.7 \%)$ patients with $\mathrm{A} 1 \mathrm{c}<7 \%$ in year 1 relapsed to $\mathrm{A} 1 \mathrm{c}>8 \%$ in year 2 .

Discussion: We estimate that the phenomenon of A1c relapse accounts for one-third of all adults identified as having uncontrolled glucose on quality measures. Proactive care strategies in high-risk patients close to goal (A1c 7-7.9\%) to help them sustain control could reduce the proportion of patients not meeting optimal A1c goals. More systematic use of patient-reported self-monitored blood glucose data could further help to identify patients who are relapsing or progressing. Further research is needed to test these hypotheses.

Keywords: diabetes, optimal A1c goals

PS2-4:

Health Coaching for Patients With Diabetes and Hypertension: Filling a Gap for Patients and Physicians

Ellis C. Dillon, ${ }^{1}$ Laura Panattoni, ${ }^{1}$ Amy Meehan, ${ }^{1}$ Judith Chuang, ${ }^{1}$ Lisa McCormick, ${ }^{1}$ Ming Tai-Seale ${ }^{1}$

\section{${ }^{1}$ Palo Alto Medical Foundation Research Institute}

Background/Aims: Health coaching is a novel primary care team-based care model using clinically supervised unlicensed providers to help patients make lifestyle changes and improve self-management of diabetes and/or hypertension. Previous health coaching programs have largely targeted indigent patients. It is unknown how privately insured patients and feefor-service providers in community practices would respond to and use health coaching services.

Methods: Findings are drawn from a study conducted at a large multispecialty clinic serving approximately 29,000 patients with diabetes or hypertension. Data were gathered 6 months after implementation of the health coach program and include observation/audio recordings of 8 health coach appointments, interviews with 8 patients, 7 referring physicians, 2 health coaches and 1 nurse practitioner clinical supervisor, and electronic health record (EHR) visit data.

Results: Out of the 2,342 patients with a diabetes- or hypertension-related primary care visit during this period, $197(12 \%)$ had health coach referrals in the EHR, and 113 $(5 \%)$ had at least one health coach visit. Patients averaged 2.5 (standard deviation: 2.6) health coach visits, but the range included 1 to 19 visits during this time period. Health coaching involved direct time spent coaching patients and indirect time spent on care management and navigation. Direct coaching ranged from biweekly in-person visits to monthly phone calls. An average in-person visit was 38 minutes (min: 18, max: 74, standard deviation: 18.9 minutes). Health coaches developed a distinct relationship with patients; they worked together to develop plans that addressed not only health concerns such as diet, exercise and monitoring of blood pressure/sugar, but also personal concerns regarding finances, family, etc. Physicians differed in the language they used to describe the health coach program to patients and in their criteria for health coaching referrals. Physicians were appreciative of health coaches as 
members of an extended care team and as liaisons between patients and services or resources.

Discussion: The role of health coaching in this primary care setting is still evolving and adjusting to meet varying levels of patient needs and preferences for contact. Health coaching fills a gap for patients and physicians who both benefit from an extended but flexible relationship that provides a new type of support.

Keywords: health coach, chronic conditions

PS2-5:

Dilated Eye Exam Compliance for Persons With Diabetes Mellitus in a Managed Care Setting

Jaejin An, ${ }^{1}$ T. Craig Cheetham, ${ }^{2}$ Fang Niu, ${ }^{2}$ Yamina Rajput, ${ }^{3}$ Adam Turpcu ${ }^{3}$

${ }^{1}$ Western University of Health Sciences; ${ }^{2}$ Kaiser Permanente Southern California; ${ }^{3}$ Genentech Inc.

Background/Aims: National practice guidelines recommend regular dilated eye examinations for persons with diabetes mellitus (DM). Regular exams can identify the presence of diabetic eye diseases leading to early detection and treatment along with vision preservation. We aimed to: 1) assess compliance with guideline-recommended dilated eye exams among persons with DM, and 2) determine the factors associated with noncompliance.

Methods: Kaiser Permanente Southern California members aged $\geq 18$ years with DM identified from January 2009 to December 2010 were followed until disenrollment or study end date (December 2013). Dilated eye exams were identified from CPT-4, ICD-9 procedure codes and retinal photographs. Compliance with guidelines over the entire duration of follow-up was the binary outcome of interest. A patient was defined as compliant when having at least one exam in each 12-month period if there was evidence of retinopathy, or at least one exam in each 24-month period if there was no evidence of retinopathy. Multivariate logistic regressions were used to investigate patient demographics and other baseline characteristics associated with noncompliance.

Results: Among the 204,073 eligible patients, mean age \pm standard deviation was $61 \pm 13$ years and $48 \%$ were female. The median follow up was 4.8 years, and overall, $71.1 \%$ of patients were compliant with dilated eye exams, including $27.7 \%$ who received an eye exam every year and $4.4 \%$ who never received a dilated eye exam. At baseline $13 \%$ of patients had retinopathy, while an additional $20 \%$ of patients developed retinopathy during follow-up time. Noncompliant patients were more likely to be younger, black, male, smokers, and have a Medicare plan, a lower income, a lower education and a higher specialist co-payment plan. In addition, these patients were less likely to be adherent to antidiabetes medications, on statin medications, take a diabetes education class and have other eye diseases; however, they were more likely to use insulin, have retinopathy, have nephropathy, and have a lower comorbidity index.

Discussion: During nearly 5 years of follow-up, $28.9 \%$ of person's with DM were noncompliant with dilated eye exam guidelines. Future research should focus on eye disease outcomes associated with noncompliance and the development of interventions to address modifiable factors associated with noncompliance.

Keywords: diabetes mellitus, risk factors

PS2-6:

Home Monitoring: Patient and Provider Perceptions of and Use of Home Blood Sugar and Blood Pressure Measurements in Primary Care Practice

\author{
Ellis C. Dillon, ${ }^{1}$ Amy Meehan, ${ }^{1}$ Judith Chuang, ${ }^{1}$ Ming Tai- \\ Seale $^{1}$
}

\section{${ }^{1}$ Palo Alto Medical Foundation Research Institute}

Background/Aims: Patients with diabetes and hypertension are increasingly asked to monitor blood glucose and blood pressure at home. Home monitoring and the resulting logs and measurements are sometimes used as a basis for adjusting treatment. Despite the importance of home monitoring, we lack an understanding of patients' ability and education about how to take accurate measurements, their perception of what to do with these measurements, and how providers use these measurements in practice.

Methods: Findings are drawn from a mixed-methods study of a primary care intervention for patients with diabetes and hypertension. The study was conducted at a large multispecialty clinic serving approximately 29,000 patients with diabetes or hypertension. Data include discussion of home monitoring in observation/audio recording of 26 primary care appointments, and interviews with 12 patients, 7 medical assistants and 9 physicians.

Results: The intervention standardized procedures for collecting patients' home blood sugar and blood pressure measurements and logging them in the electronic health record. However, there was no standardization of the procedures or tools patients used to collect this data. Patients described various motivations for home monitoring, ranging from physician requests to adjusting daily insulin dosage. Several patients were uneducated about how to take measurement, e.g. what "fasting blood sugar" meant. Some patients were unsure what to do if measurements were too high or too low. Other patients lacked training and reported problems using devices. Physicians often asked patients to call in/email measurements after appointments or medication changes. Increasingly physicians appear to be using these home measurements for clinical decision-making, yet the reliability of these measurements may vary widely.

Discussion: Home monitoring offers an opportunity to further engage patients in their care and provides an opportunity to help them better understand how blood pressure and blood sugar affects their health. However, patients exhibit varying strategies of when, how and why to check their blood sugar and blood pressure. Relying on patient-collected data may result in problematic clinical decisions, and there is a need for consistent and early patient education to ensure accurate home monitoring and steps to take if numbers are either too high or too low.

Keywords: diabetes, hypertension 
PS2-7:

\section{Building an Interactive Platform for Physical Activity Coaching}

Angelika Clarke, ${ }^{1}$ Annette R. Gregson, ${ }^{1}$ Suzanne Furuya, ${ }^{2}$ Vincent Phung, ${ }^{1}$ Ricardo Yosy, ${ }^{1}$ Chris Foo, ${ }^{1}$ Huong Q. Nguyen ${ }^{1}$

\section{${ }^{1}$ Kaiser Permanente Southern California; ${ }^{2}$ Kaiser Permanente Northern California}

Background/Aims: Patients with chronic obstructive pulmonary disease (COPD) who are physically inactive have worse outcomes. The evidence is unequivocal that intensive supervised exercise training as part of pulmonary rehabilitation, a guideline-recommended therapy, improves symptoms, physical functioning and quality of life and reduces hospitalizations for COPD exacerbations. However, patient participation in supervised exercise at center-based programs is very low, which undermines the wide scale adoption of this approach in real-world clinical settings for large numbers of patients. Alternative models to center-based rehabilitation that are more patient-centered, scalable and sustainable are needed in order to reach patients who otherwise would not have other options. The aim of this project was to develop an integrated informatics architecture to support the implementation of a patient-centered, technology-enabled physical activity coaching intervention model (Walk On!) for patients with COPD.

Methods: A multidisciplinary team of researchers, clinicians, innovation consultants and software developers, as well as patient end users, embarked on an iterative design process over the course of 6 months to build out three key components of the Walk On! platform. An interactive voice response (IVR) module was developed to collect physical activity and symptom data from patients who do not have access to the Internet. An existing vendor supported web-based physical activity application paired with a wireless sensor was modified for use by patients with Internet access. Finally, an interactive web-based dashboard was created to visually summarize the integrated physical activity and symptom data from the IVR and web-based systems for the activity coaches to review patients' progress and conduct targeted outreach to support patients' efforts to be physically active.

Results: We have completed the IVR module build and are close to completing the enhancements to the physical activity web-based application. Building the dashboard that integrates data from these two sources has required significant effort, especially the user-interface, coding the dynamic functions and building the data back-end. The platform will be pilot tested on 30 patients starting in December 2014.

Discussion: We will present data from our pilot at the HMORN meeting. The Walk On! platform is designed to be usable, scalable and generalizable.

Keywords: COPD, intervention

PS2-9:

Health Outcome Effects of Common Medications in Elders With Multiple Conditions
Heather G. Allore, ${ }^{1}$ Gail McAvay, ${ }^{1}$ Mary Tinetti ${ }^{2}$

\section{${ }^{1}$ Yale New Haven Hospital; ${ }^{2}$ Yale University Hospital}

Background/Aims: Determining medication effects is more complex in individuals with multiple chronic conditions (MCC). One approach to addressing these limitations is to define effectiveness through the use of cross-condition, universal health outcomes such as self-reported health (SRH). Appropriate methodology is needed to evaluate medication effects in the setting of MCC.

Methods: We studied 9 commonly used oral medications from national disease guidelines (renin-angiotensin system blockers (RAS), statins, thiazides, calcium channel blockers, selective serotonin reuptake inhibitors, metformin, warfarin and clopidogrel) recommended for 8 common chronic conditions (atrial fibrillation, coronary artery disease, depression or anxiety, diabetes mellitus, heart failure, hyperlipidemia, hypertension and pulmonary embolism/ venous thrombosis) and used by at least $20 \%$ of 8,517 Medicare Current Beneficiary Survey enrollees with two or more MCC from 2005-2009 with follow-up data available through 2011. We estimated the odds of high SRH (good-excellent) of the most commonly used medications for 8 common and morbid chronic conditions, adjusted for 14 covariates and accounting for within-subject correlation. For absolute population level estimates, we applied the longitudinal extension of the average-attributable-fraction with time-varying conditions on recurrent SRH.

Results: The most common dyads of conditions at baseline were hypertension and hyperlipidemia, with $71.3 \%(6,073$ of 8,517 ). On average, $11.3 \%$ (96 of 8,517 ) discontinued a medication over the 3-year follow-up period, whereas $6.9 \%(588$ of 8,517$)$ started a new medication. All the conditions except atrial fibrillation were significantly associated with poorer SRH; pulmonary embolism/venous thrombosis had borderline significance. Hyperlipidemia had significantly higher odds of high SRH. There were four significant condition-medication interaction terms. Regarding participants with hypertension, the odds of high SRH for people who take RAS blockers were greater than those who do not. Conversely, the odds of high SRH for people who take thiazide for hypertension are lower than those who do not. The odds of reporting high SRH for people taking statins is higher than those not taking statins within the hyperlipidemia subpopulation. The odds of high SRH among people who have coronary artery disease is lower in those who take clopidogrel than those who do not.

Discussion: Medication effects on universal health outcomes provide a way to compare across conditions.

Keywords: aging, multiple chronic conditions

PS2-10:

Identification of Multiple Chronic Conditions That Yield the Highest Impact of Cognitive Screening

Leah R. Hanson, ${ }^{1}$ Terry R. Barclay, ${ }^{1}$ Ann M. Hanson, ${ }^{1}$ Logan H. Stuck, ${ }^{1}$ Jean M. Crow, ${ }^{1}$ Michael H. Rosenbloom ${ }^{1}$ 
${ }^{1}$ HealthPartners

Background/Aims: Diabetes mellitus (DM), hypertension (HTN) and hyperlipidemia (HL) are common geriatric chronic diseases that may increase dementia risk. Screening for cognitive impairment is not currently recommended due to lack of studies demonstrating benefit. HealthPartners piloted using the Mini-Cog to screen patients aged $\geq 65$ years, and physicians expressed concern about the feasibility of screening all patients. In order to identify subgroups of patients who may potentially benefit most from cognitive screening, we examined screen fail rates, retrospective health care utilization and the impact of multiple chronic conditions in this cohort.

Methods: Data from the 18 months prior to cognitive screening was collected from the electronic medical record and included the Mini-Cog score (scored 0-5, fail is less than 4), demographics, presence of diagnosis for three chronic diseases, and measures of health care utilization. The cohort was divided into eight subgroups: DM alone, HTN alone, HL alone, DM+HTN, DM+HL, HTN+HL, DM+HTN+HL, and no chronic conditions. Utilization outcomes were analyzed using Poisson regression accounting for age and sex; the DMonly model was not useable due to a small sample size.

Results: The mean age of the cohort $(\mathrm{N}=1,124)$ was 77 years (41\% male), and the overall cognitive screen fail rate was $32 \%$. Fail rates were highest in the small DM group $(50 \%$ fail), followed by patients lacking the three chronic conditions (36\% fail), and lowest in the DM+HL (21\%) and HTN (26\%) cohorts. Overall, patients failing the screen had a significantly higher incidence rate of hospitalization (23\%) and emergency room visits (42\%). This result was driven by two subgroups of multiple chronic conditions, DM+HTN and DM+HTN+HL, with those failing having significant increases in rates of hospitalization (535\% and 56\%, respectively) and emergency room visits $(252 \%, 102 \%)$.

Discussion: Based on higher retrospective utilization, patients with $\mathrm{DM}+\mathrm{HTN}$ and $\mathrm{DM}+\mathrm{HTN}+\mathrm{HL}$ may benefit most from cognitive screening. Surprisingly, higher screen fail rates were found in patients without DM, HTN or HL, which may be partially explained by a lower rate of clinic visits (mean 0.98 visits per month vs. 1.33). Prospective longitudinal studies are necessary to better support the role of the screening in specific groups of patients with chronic conditions.

Keywords: aging, multiple chronic conditions

PS2-11:

The Group Health-University of Washington Adult Changes in Thought Study: A Living, Learning Laboratory for Aging and Multiple Chronic Conditions Research

Eric B. Larson, ${ }^{1}$ Paul K. Crane, ${ }^{2}$ Erin J. Bowles, ${ }^{1}$ Rod L. Walker, ${ }^{1}$ Melissa L. Anderson, ${ }^{1}$ Darlene White, ${ }^{1}$ KatieRose Richmire, ${ }^{1}$ Andrea LaCroix, ${ }^{3}$ Susan M. McCurry, ${ }^{2}$ Linda Teri, ${ }^{2}$ Shubhabrata Mukherjee, ${ }^{2}$ Thomas J. Montine ${ }^{2}$

${ }^{1}$ Group Health Cooperative; ' $U$ niversity of Washington; ${ }^{3}$ University of California San Diego
Background/Aims: Delivery system-based research can meet today's need for practical clinical evidence and provide opportunities for discovery in everyday populations, particularly for an increasing number of people with multiple chronic conditions. The evolution of the Adult Changes in Thought (ACT) study, a long-standing partnership between Group Health and University of Washington, demonstrates the value and lessons learned from a living laboratory on aging.

Methods: We responded to a 1986 National Institute of Aging request for Alzheimer's disease patient registries to identify people with incident Alzheimer's disease (AD) at Group Health. Partnering with University of Washington allowed us to evaluate candidate genetic markers and develop a biobank. In 1994, we established the ACT study, a cohort of randomly selected people over age 65 without dementia. We initially recruited 2,581 participants, followed by an expansion cohort of 811 , and a continuous replacement sampling strategy to replace participants who die, become demented or are lost to follow-up. We maintain a constant cohort of approximately 2,000 living persons who are followed every two years.

Results: ACT has enrolled over 5,000 subjects, including over 1,000 cases of incident dementia (over $70 \%$ AD) and almost 600 autopsy cases, of which about half have extensive frozen tissues with a rapid autopsy protocol. Our biobank includes extensive genome-wide single nucleotide polymorphism, exome sequence and gene expression data. Our population-based neuropathology biobank is unique worldwide. Our collaboration started a learning laboratory for pragmatic trials, and contributed to the development of the Seattle Protocols for dementia care, descriptive studies of health services utilization, and widely accepted risk factor and outcome data for persons with AD. ACT has served as the parent grant for numerous studies involving genetics, neuroimaging, pharmacoepidemiology, neuropathology, traumatic brain injury, treatment trials, methods development and career development.

Discussion: Studies of important age-related conditions will provide valid research results if based on a representative population. The ACT study created a platform for a populationbased living laboratory on aging across a wide range of disciplines and scientific inquiry. Effective partnerships, including widespread data and specimen sharing, are foundational and critical for optimal success.

Keywords: aging, multiple chronic conditions

\section{COMMUNICATION/DISSEMINATION/ IMPLEMENTATION}

\section{B1-5: \\ Cluster Randomized Trial of Enhanced Versus Standard Implementation Strategy to Improve Collaborative Care Uptake and Patient-Level Utilization Outcomes}

Jeanette A. Waxmonsky, ${ }^{1}$ Amy K. Kilbourne, ${ }^{2}$ David E. Goodrich, ${ }^{2}$ Hyungjin M. Kim, ${ }^{3}$ Lilia Verchinina, ${ }^{3}$ Zongshan Lai, ${ }^{2}$ Daniel Eisenberg, ${ }^{3}$ Julia T. Kyle, ${ }^{3}$ Kristina M. Nord ${ }^{2}$ Jenny Han, ${ }^{1}$ Mark S. Bauer, ${ }^{4}$ Marshall R. Thomas ${ }^{1}$ 
${ }^{1}$ Colorado Access; ${ }^{2}$ University of Michigan Medical School; ${ }^{3}$ University of Michigan; ${ }^{4}$ Veterans Administration Boston Healthcare System

Background/Aims: Implementation strategies are increasingly being used to promote the uptake of evidencebased practices, yet few studies have demonstrated their impact on the efficiency of patient-level utilization. This cluster randomized controlled study determined whether an enhanced versus standard version of the Replicating Effective Programs (REP) implementation strategy, designed to promote the uptake of an evidence-based collaborative care program, impacted emergency department (ED) utilization outcomes among patients with bipolar disorder. We hypothesized that the enhanced version would lead to decreased ED use compared to the standard.

Methods: Seven community-based clinics in Michigan and Colorado were randomized to receive either standard (REP) or enhanced REP (E-REP) to improve the uptake of Life Goals Collaborative Care (LG-CC). Providers at sites randomized to REP received the LG-CC toolkit (manual, implementation guide), LG-CC training and, as needed, technical assistance. Providers at sites receiving E-REP were given support to customize LG-CC manuals and training and ongoing facilitation for 6 months, where organizational barriers to LG-CC uptake were addressed through provider coaching to support integration of LG-CC into routine care. Providers were responsible for implementing four weekly LG-CC group sessions and monthly care management calls over 6 months with patients with a diagnosis of bipolar disorder seen at their clinics. Prior to attending LG-CC sessions, patients were consented and completed baseline and follow-up assessments at 6 and 12 months. Primary utilization outcomes included self-reported ED visits.

Results: Within 12 months, patients $(\mathrm{N}=246)$ at sites randomized to E-REP $(\mathrm{n}=146)$ compared to REP sites $(n=100)$ had a smaller self-reported number of ED visits (0.14 for E-REP sites vs. 0.36 for REP; odds ratio: 0.24, $\mathrm{P}=0.004$ ) adjusted for patient-level demographics (sex, race, age, education, homelessness, employment), clinical factors (psychiatric symptoms, medical diagnoses), ED utilization measured in the baseline survey and number of LG-CC contacts. LG-CC uptake, particularly having at least 4 care management contacts, appeared to mediate the effect of the E-REP implementation strategy and decreased ED use.

Discussion: Implementation strategies such as enhanced REP that provide customization and coaching in the use of a collaborative care model may reduce ED use, primarily by improving overall uptake and increasing care management contacts.

Keywords: implementation strategies, patient-level outcomes utilization

B4-3:

Interviews With Patients Offered Lung Cancer Screening in Primary Care: Lessons in How Screening May Influence Smoking Cessation
Steven B. Zeliadt, ${ }^{1}$ Jaimee L. Heffner, ${ }^{2}$ Deborah E. Klein, ${ }^{3}$ George Sayre,${ }^{4}$ Lynn F. Reinke, ${ }^{4}$ Shuva Dawadi, ${ }^{5}$ David H. Au ${ }^{4}$

${ }^{1}$ Group Health Cooperative; ${ }^{2}$ Fred Hutchinson Cancer Research Center; ${ }^{3}$ Swedish Medical Group; ${ }^{4}$ Veterans Health Administration; ${ }^{5}$ University of Washington

Background/Aims: We interviewed current smokers offered lung cancer screening as part of a demonstration project to explore how the availability of screening and the receipt of screening results, including both normal and abnormal findings, potentially influences patients' beliefs about the consequences of smoking and motivations around smoking cessation.

Methods: We conducted semistructured interviews with 37 patients identified from 7 medical centers across the United States who were identified as eligible for lung cancer screening and offered screening by their primary care provider as part of the Veteran Health Administration's (VHA) Lung Cancer Screening Clinical Demonstration Project. Data analysis was conducted concurrently with data collection using inductive and deductive content analysis.

Results: A total of 37 current smokers participated in interviews from among 186 invited screenees purposively sampled from the Demonstration Project between April and August 2014; $11 \%$ were female and $27 \%$ non-Caucasian. Four patients (11\%) declined screening, and 9 of $30(30 \%)$ patients who were screened during the study period were identified with a nodule finding $<1 \mathrm{~cm}$. We identified four themes related to smoking cessation behaviors. Screening, when offered by their primary care provider, was very appealing to many patients in contrast with "badgering" by providers about smoking cessation and descriptions of futility associated with prior quit attempts. Screening was associated with reflection and personalization about harms of smoking; however, sometimes contemplation about screening reinforced misperceptions about risks of smoking or exaggerated negative effect and worry. Patients with normal findings often interpreted the result as evidence that smoking hasn't harmed them. Multiple patients with abnormal findings described the belief that detection of nodules meant they were being helped by technology. These patients described trusting their doctors and "watching and waiting" as the way to avoid chemo and unpleasant treatment for lung cancer, believing they can put off any action, including quitting, until there is more evidence of growth.

Discussion: We identified several concerning pathways in which screening, when offered as part of routine care and described as having proven efficacy, may negatively influence cessation. Providers should be aware of these pathways and tailor discussions to ensure screening does not lower motivations around cessation.

Keywords: lung cancer screening, smoking cessation

D3-4:

The Role of Cost Information in Health System Decisions to Adopt New Services 
Frances L. Lynch, ${ }^{1}$ Gregory Clarke, ${ }^{1}$ Jennifer Schneider, ${ }^{1}$ Alison J. Firemark, ${ }^{1}$ Megan M. Scheminske ${ }^{1}$

\section{${ }^{1}$ Kaiser Permanente Northwest}

Background/Aims: Health systems are under increasing pressure to make careful choices about which new services to adopt or encourage for their populations. At the same time, explicit use of information on cost-effectiveness of interventions in the decision process is controversial in the United States. Yet health system decision-makers routinely need to make decisions about what types of interventions to adopt or promote within a limited budget. Economic evaluations such as cost-effectiveness analyses are one source of information that could potentially aid health system decision-makers in evaluating interventions. Yet little is known about how useful typical published economic evaluations are to health system decision-makers, or whether or not they provide the type of information most useful in the decision process.

Methods: We conducted in-depth qualitative interviews by telephone with a purposive sample of 38 public and private health system decision-makers. We discussed decisionmaking in general, type of information used in the process and the usefulness of cost information. All transcripts were coded by trained coders and entered into Atlas.ti, which was used to code and manage data and to generate reports for analysis. The team reviewed reports and engaged in an iterative process of discussion and review, resulting in the final themes. We also looked at results by size of organization and type of service under evaluation (medical or mental health).

Results: Participants reported a variety of internal and external factors perceived to influence adoption to their organizations. The two most highly endorsed factors were clinical effectiveness and financial feasibility. Other highly endorsed factors included ease of integration into current practice and provider/staff acceptability. Most participants reported wanting information on direct costs of an intervention, and about half of the participants wanted published costeffectiveness information. Participants reported differences in decision-making between medical and mental health interventions, for example, several participants suggested that mental health interventions were more difficult to evaluate both in terms of effectiveness and cost compared to medical interventions.

Discussion: Cost information is important to all the organizations and decision-makers we interviewed. However, typical published economic evaluations may not contain some of the cost information needed by decision-makers.

Keywords: cost-effectiveness, implementation

PD1-7:

System Alignment for VaccinE Delivery (SAVED): A Technology-Based Intervention to Improve Influenza and Pneumococcal Vaccination

Sarah L. Cutrona, ${ }^{1}$ Larry Garber, ${ }^{2}$ Lloyd Fisher, ${ }^{1}$ Peggy Preusse, ${ }^{3}$ Sarah L. Goff, ${ }^{4}$ Meera Sreedhara, ${ }^{2}$ Madeline Jackson, ${ }^{2}$ Devi Sundaresan, ${ }^{3}$ Kathleen M. Mazor ${ }^{2}$
${ }^{1}$ University of Massachusetts Medical School; ${ }^{2}$ Meyers Primary Care Institute; ${ }^{3}$ Reliant Medical Group; ${ }^{4}$ Baystate Medical Center

Background/Aims: Influenza and pneumococcal vaccines are beneficial but underutilized. Using health information technology and patient self-reported data within our Epic electronic health record (EHR), we aim to improve rates of influenza and pneumococcal vaccination in a large multispecialty group practice in central Massachusetts.

Methods: We have undertaken a multifaceted vaccine promotion program with patient-, provider- and systemlevel components. We conducted patient, physician, nurse and medical assistant qualitative interviews to inform development of provider educational materials and patient outreach materials. Provider education was delivered via inperson brief presentations at practice sites given by research team physicians (respected members of the group practice). Patient outreach will target patients eligible but overdue for influenza vaccine (some are also overdue for pneumococcal vaccine). In November 2014, we will randomize 20,000 patients who are active users of electronic patient portals to (a) receipt of a portal message providing education and access information on flu and pneumococcal vaccines, (b) receipt of an interactive voice recognition (IVR) call with similar content, (c) both, or (d) neither (usual care). Patients without electronic portals $(10,000$ patients) will be randomized to (a) receipt of IVR call, or (b) usual care. Both outreach methods will invite patients to report receipt of flu vaccines outside of the medical group and will automatically update the EHR with self-reported vaccine completion (increasing accuracy of existing EHR provider alerts). Both methods will (a) use questionnaires to elicit barriers for unvaccinated patients, and (b) deliver information targeted to patient concerns.

Results: We are using multilevel interventions and technological innovation to increase rates of recommended vaccine completion in our population and to improve the accuracy of existing provider-directed EHR vaccine alerts. To date, factors contributing to success include (a) research team's inclusion of physician thought-leaders well-known within the practice; and (b) alignment of study goals with the mission of the group practice (improving delivery of preventive care, improving accuracy and efficiency of EHR without interfering with workflow, and achieving meaningful use goals by inviting patient communication via e-portals).

Discussion: Aligning our research with the existing goals and values of the medical group practice has yielded effective collaborations and facilitated project success.

Keywords: flu vaccine, patient portal

PS1-10:

Implementation of an Evidence-Based Breast Cancer Support Tool for Newly Diagnosed Breast Cancer Patients as Standard Care at Two Institutions: Use and Sustainability

Alanna Kulchak Rahm, ${ }^{1}$ Erica F. Morse,${ }^{2}$ Helene McDowell, ${ }^{3}$ Breanne Barela, ${ }^{2}$ Jana Bolduan Lomax, ${ }^{4}$ Liza Reifler, ${ }^{2}$ Suzanne Pingree, ${ }^{3}$ James Dearing, ${ }^{5}$ Robert Hawkins ${ }^{3}$ 
${ }^{1}$ Geisinger Health System; ${ }^{2}$ Kaiser Permanente Colorado; ${ }^{3}$ University of Wisconsin-Madison; ${ }^{4}$ Exempla St. Joseph Hospital; ${ }^{5}$ Michigan State University

Background/Aims: The Comprehensive Health Enhancement Support System (CHESS) was created to answer the need for information and support and to improve the quality of life of breast cancer patients. CHESS has been validated in several randomized trials. The aim of the current study is to implement CHESS as part of standard care at two Denver health care systems. All women who received a breast cancer diagnosis were offered access to CHESS during or very shortly after notification of their diagnosis and throughout the treatment process to provide information and decision-making support. Methods: From July 2012 to July 2013, all newly diagnosed breast cancer patients at both institutions were offered CHESS. Two months postdiagnosis, CHESS users and non-users were randomized to no contact or to complete a telephone survey or interview about CHESS. Patient use of CHESS was tracked on the system server. In-depth interviews also were conducted with providers of both institutions after CHESS had been in use for one year. Providers were asked about organizational changes affecting CHESS implementation and sustainability, and potential to sustain CHESS after study completion.

Results: All patients were offered CHESS at least once, most often by nurses responsible for delivering the diagnosis. Overall, $24 \%$ of patients used CHESS by 2 months postdiagnosis. Users who liked CHESS said it was a safe and structured place to get needed information. For others, providing a username and password was a barrier to CHESS use. Providers liked having the CHESS resource to give patients; however, some felt that resources required for sustainability might be best used elsewhere. Other providers felt that CHESS should be sustained and expanded throughout each organization because of the potential impact for the women who preferred to use web-based resources. Providers also felt that no web-based resource could replace individual interactions with patients.

Discussion: Patients who liked CHESS felt it was helpful and appreciated the resource. Providers liked having CHESS to offer patients. Some providers felt CHESS should be maintained and expanded, while others felt it was no longer unique. Various resources may be necessary to meet patient and provider needs.

Keywords: implementation, breast cancer

PS1-19:

Barriers to Implementing and Disseminating an Intervention to Improve Hypertension Control With Home Monitoring and Uploading of Data Into an Electronic Health Record

Barry G. Saver, ${ }^{1}$ Jenna Marquard, ${ }^{2}$ Lawrence Garber, ${ }^{3}$ Brian D. Amster, ${ }^{2}$ Peggy Preusse, ${ }^{3}$ DJ Gove ${ }^{3}$
${ }^{1}$ University
of Massachusetts
Medical
${ }^{2}$ University of Massachusetts Amherst;
School;
Primary Care Institute

Background/Aims: The CONtrolling Disease Using Inexpensive Technology (CONDUIT) study tests an approach to monitoring and managing hypertension that could be easily and widely disseminated and scalable to self-monitoring of other conditions.

Methods: The CONDUIT randomized control trial tests whether an intervention consisting of self-monitoring of blood pressure (BP) and a feedback loop involving nurses and primary care providers improves control of hypertension in patients with uncontrolled hypertension. Rather than embedding the intervention in a proprietary electronic health record (EHR), we used the free Microsoft HealthVault platform to receive participants' BP readings electronically and developed an interface to transmit HealthVault data into the EHR. To further maximize dissemination potential, participants who could not upload BP data from home could upload data at their clinics.

Results: Substantial effort was required to develop the HealthVault-EHR interface, including custom programming to poll HealthVault for BP data and periodically send messages to nurses summarizing the patient-uploaded data. We encountered barriers to system implementation at multiple levels, often because vendors did not consider use cases similar to ours. For instance, BP monitors lacked unique device identifiers; neither the devices, HealthVault or the EHR validated date/time data; software changes by any of several entities caused data flows to break and required frequent revision of patient instructions; and protected health information protection in clinic-based uploads proved challenging. Patients and staff expressed satisfaction when the system worked, but had limited tolerance for software failures. Most clinicians supported the system, but would have greater enthusiasm if patient-generated BP readings were considered in HEDIS scoring.

Discussion: When the CONDUIT system worked as designed, it was well-accepted by patients and providers, but the various "moving parts" under control of different organizations led to multiple challenges and frustrations. For similar interventions to be successful, hardware and software vendors must consider a wider range of use cases in their design processes.

Keywords: hypertension, electronic health record

PS2-17:

A Real-World Approach to a Value-Based DecisionMaking Framework for Genetic Testing

Susan R. Snyder, ${ }^{1}$ James M. Pitcavage, ${ }^{1}$ Jonathan A. Bock $^{1}$

${ }^{1}$ Geisinger Health System

Background/Aims: Relatively few genetic tests have been formally evaluated for cost-effectiveness, and many lack widely accepted evidence of clinical utility. Pragmatic approaches are needed to support real-world, value-based decision-making for genomic-based screening and treatment strategies, including patient selection criteria to identify those with greater potential to benefit. The lack of evidentiary standards for clinical implementation as well as evidence 
gaps are barriers to decision-making for implementation and reimbursement. Value-improving technology impacts clinical decisions by improving patient care without a disproportionate increase in overall costs. Available knowledge about genomic testing and potential concerns can be integrated and applied using a process to assess value for patient care. The objective of this research is to develop a decision-making framework to support implementation of value-improving technology. Satisfying this objective should avoid premature use of tests that provide little benefit or pose significant health risks compared to usual care.

Methods: Multicriteria decision analysis (MCDA) provides structure and transparency in evaluating options by considering the relative importance of different criteria using a weighting scheme and information on each option's performance. The results of the review of health care MCDA methods are synthesized and applied to systematically identify and structure elements of value. We then integrate and evaluate clinical, epidemiological and economic evidence to assess the value of genetic and pharmacogenomic testing to support real-world decision-making.

Results: A model with relative weighting for all elements of measured value is developed and applied to facilitate aggregating measures to support real-world decision-making. Current knowledge about genetic and pharmacogenomic testing is integrated and applied to move from the concept of value to making a decision. This is done by measuring and gathering evidence on each of the elements and aggregating the combined elements, with uncertainty of an outcome treated as an element of value.

Discussion: Assuming a continuing shortage of formal and direct evidence for genetic testing, transparent consideration of available, including indirect, evidence can be used to assess whether outcomes relative to cost are likely to offer sufficient value in a comprehensible and actionable decision-making framework. This framework offers the potential to reduce barriers to widespread implementation.

Keywords: decision-making, personalized genomic medicine

PS2-21:

\section{Community of Evaluation Practice Across HMORN Sites}

Cristy R. Geno Rasmussen, ${ }^{1}$ Erica F. Morse,${ }^{1}$ Carmen Luna, ${ }^{1}$ Heather Nuanes, ${ }^{1}$ Michelle Henton, ${ }^{1}$ Lisa Harner, ${ }^{1}$ Breanne Barela ${ }^{1}$

\section{${ }^{1}$ Kaiser Permanente Colorado}

Background/Aims: Kaiser Permanente's Community Health Initiative (CHI) strives to foster healthy eating and active living (HEAL) policy and environmental change to promote obesity prevention across communities it serves. The $\mathrm{CHI}$ evaluation framework uses a logic model that factors in baseline conditions, existing community assets, Kaiser Permanente assets, and design principles toward effective interventions and community capacity building strategies. Kaiser Permanente and the Center for Community Health and Evaluation (CCHE) at Group Health use evidence- based community health partnership characteristics to guide the evaluation of $\mathrm{CHI}$ initiatives using four primary design principles: place-based focus, multilevel interventions, multisectorial collaboration, and community engagement and ownership. The aims of the study are to (1) assess the generalizability of the CHI evaluation framework across HMORN sites, and (2) evaluate the application of the design principles as they apply to the multilevel evaluation structures across HMORN sites toward future evaluation collaborations within HMORN.

Methods: In an effort to understand the variability in evaluation experience and the generalizability of evaluation practice across HMORN sites, semistructured interviews were conducted with HMORN partners to explore the key design principles, including: (1) place-based focus to examine geographic variability across HMORN sites and communities, (2) multilevel interventions to explore evaluation activities across HMORN sites and communities, (3) multisectorial collaborations to examine how evaluation strategies are used differently across HMORN sites, and (4) community engagement and ownership to understand how evaluation teams partner within HMORN sites.

Results: Evaluation lessons learned to date are shared and may be used to inform multilevel HMORN evaluation collaborations in the future. Findings support the development of an evaluation inventory to understand common methods, metrics and measurements as they are used across HMORN sites.

Discussion: By gaining a better understanding of how evaluation practices are developed and informed, efforts can be made to improve partnerships toward more effective evaluation efforts and collaborations within HMORN.

Keywords: evaluation, organizational practice

\section{GENETICS/BIOREPOSITORY}

C1-1:

The Kaiser Permanente Biobank: A Multiregion Resource Linking Specimens and Electronic Medical Records for Broad Research in an Integrated Health Care Delivery System

Heather S. Feigelson, ${ }^{1}$ Lisa A. Croen, ${ }^{2}$ Katrina A. Goddard, ${ }^{3}$ Stacey A. Honda, ${ }^{4}$ Michael A. Horberg, ${ }^{5}$ Corinna Koebnick, ${ }^{6}$ Ashli Owen-Smith, ${ }^{7}$ Sarah Rowell, ${ }^{2}$ Catherine Schaefer, ${ }^{2}$ Carol P. Somkin, ${ }^{2}$ Karen M. Emmons $^{2}$

${ }^{1}$ Kaiser Permanente Colorado; ${ }^{2}$ Kaiser Permanente Northern California; ${ }^{3}$ Kaiser Permanente Northwest; ${ }^{4}$ Kaiser Permanente Hawaii; ${ }^{5}$ Mid-Atlantic Permanente Research Institute; ${ }^{6}$ Kaiser Permanente Southern California; ${ }^{7}$ Kaiser Permanente Southeast

Background/Aims: Kaiser Permanente (KP) is an integrated health care delivery system spanning seven geographic regions with 9.3 million members. The KP Biobank is an extension of existing biobanks in four KP regions - Northwest, Southern California, Georgia and Northern California — which 
together include specimens and phenotypic data for a total of 210,000 members. This includes the Research Program on Genes, Environment, and Health (RPGEH) Biobank, and the Genetic Epidemiology Research on Adult Health and Aging (GERA) cohort, which has genome-wide genotype data on 110,266 participants. We describe the design and features of a new initiative to consolidate these efforts into a single entity, called the KP Biobank, and expand the cohort to a total of 500,000 participants across all seven KP regions.

Methods: The KP Biobank will include a general cohort (410,000 participants), designed to represent the diversity of KP members, and two specialized cohorts: a cancer cohort $(30,000$ participants) and a pregnancy cohort $(60,000$ participants). For the cancer cohort, we will develop a rapid cases ascertainment system to identify and enroll cases close to the time of diagnosis. For the pregnancy cohort, newly pregnant members will be asked to provide specimens during their first and second trimesters of pregnancy. Whole blood and serum will be collected from participants in every cohort. Participants consent to broad future uses of specimens and data. Specimens will be stored centrally at an existing facility in Northern California and linked to phenotypic information from the medical record. We also will collect patient reported information on demographics, behavioral and environmental exposures via survey. A unique feature of the KP Biobank is the establishment of a translational research center (TRC), with a mission to support clinical integration of new discoveries and research across the translational continuum. The consolidated governance of the KP Biobank will streamline and facilitate collaboration and partnerships through a single access review committee and application procedure. Any researcher, including external scientists, may apply for use of specimens or genotyping data.

Results: Not applicable.

Discussion: The KP Biobank will facilitate future genomic and nongenomic research, particularly questions addressing diverse populations, longitudinal measures to assess changes over time and long-term outcomes.

Keywords: biorepository, genetics

\section{HEALTH CARE DISPARITIES}

\section{A2-1:}

Do Clocks Move at Different Speeds? Cultural Variation in the Satisfaction of Wait Time in Outpatient Clinics

Sukyung Chung, ${ }^{1}$ Nicole E. Johns, ${ }^{1}$ Latha Palaniappan, ${ }^{2}$ Harold S. Luft ${ }^{1}$

${ }^{1}$ Palo Alto Medical Foundation Research Institute; ${ }^{2}$ Stanford University School of Medicine

Background/Aims: Asians report low levels of satisfaction regarding wait time. Expectations about wait time based on cultural norms may contribute to this low satisfaction. We examined drivers of racial/ethnic differences in satisfaction in wait time by comparing subjective satisfaction scores to patient-reported and objective electronic health record (EHR)- recorded wait times.

Methods: We paired patient satisfaction survey data with EHR-recorded actual wait times, visit characteristics and patient demographics. The study population is comprised of patients of a large multispecialty ambulatory care organization in Northern California from 2011 to 2013. Patient satisfaction surveys were mailed to patients after randomly selected clinic visits. We focused on responses from non-Hispanic white (NHW), Asian and Latino patients $(n=213,375 ; 80.6 \%$ of 264,781 total responses). Individual survey responses were linked to visit information including wait time in the EHR. Patient demographics (age, sex, English proficiency), visit characteristics (scheduled appointment time, appointment length, time of a day, whether own PCP visit, number of years with the PCP, number of days between visit and survey return), and physician fixed effects were controlled in the multivariate analyses.

Results: After controlling for demographic and visit characteristics, all Asian subgroups reported substantially lower levels of satisfaction with wait time and longer perceived wait time compared to NHW. This trend persisted after further adjusting for EHR-recorded actual wait time. More Chinese and Asian Indian patients (the majority of Asian patients) arrived to the clinic closer to or slightly later than their scheduled appointment time compared to NHW, but their total wait time at the waiting room was shorter. Japanese and Filipinos, on the other hand, arrived sooner and waited longer than NHW. No noticeable difference was observed in wait time measures between Latino and NHW patients.

Discussion: Wait time in clinic is an objective measure that is assessed subjectively in patient satisfaction surveys. The persistent lower satisfaction and longer perceived wait time among Asians suggests that Asians have different expectations regarding wait times. Different cultural norms regarding wait time among Asians should be taken into account to provide culturally competent patient-centered services to a diverse population.

Keywords: wait time, patient-centered care

A2-4:

Is "Asian" a Meaningful Category for Studying and Reporting Health and Health Care Disparities? A Comparison of Filipino and Chinese Kaiser Permanente Northern California Members Aged 2579 on Health Status, Selected Health Behaviors, and Use of the Patient Portal

\section{Nancy P. Gordon ${ }^{1}$ \\ ${ }^{1}$ Kaiser Permanente Northern California}

Background/Aims: While the broad category "Asian" is often used for health disparities studies and monitoring, results may not meaningfully describe a race/ethnicity, but rather mask important differences across Asian subgroups. To exemplify this, Kaiser Permanente Northern California (KPNC) Filipino and Chinese health plan members were compared on demographic and health characteristics and patient portal use. 
Methods: Data from the 2008-2011 KPNC Member Health Surveys, 2011 KPNC disease registries and 2011 kp.org utilization were analyzed for Filipinos and Chinese aged 25-64 (non-senior) and 65-79 (senior) years. All differences listed are significant at $\mathrm{P}<0.05$ by t-test.

Results: Compared to Chinese, non-senior Filipinos were less likely to be college graduates ( $57.2 \%$ vs. $73.6 \%)$, more likely to have incomes $\leq \$ 25,000$ (non-seniors: $7.4 \%$ vs. $4.3 \%$; seniors: $32.8 \%$ vs. $17.1 \%$ ) and less likely to have incomes over $\$ 80,000$ (non-seniors: $42.8 \%$ vs. $58.6 \%$; seniors: $16.8 \%$ vs. $32.2 \%$ ). Filipinos were more likely to be diabetic (nonseniors: $14.4 \%$ vs. $5.5 \%$; seniors: $38.8 \%$ vs. $21.4 \%$ ) and hypertensive (non-seniors: $31.4 \%$ vs. $14.7 \%$; seniors: $78.2 \%$ vs. $61.6 \%$ ), and seniors were less likely to report excellent/ very good health $(31.3 \%$ vs. $42.3 \%)$. Filipino non-seniors were more likely to be smokers $(10.3 \%$ vs. $5.0 \%)$ and more likely to sleep $<6$ hours/day ( $14.4 \%$ vs. $6.2 \%)$. Filipinos aged 25-79 were less likely to consume $3+$ servings fruit/vegetables daily (women: $26.1 \%$ vs. $50.6 \%$; men: $18.6 \%$ vs. $37.8 \%$ ), more likely to be obese $(15.4 \%$ vs. $6.9 \%)$ and less likely to believe health behavior risks can greatly impact health (nonseniors: $68.3 \%$ vs. $82.6 \%$; seniors: $59.9 \%$ vs. $71.9 \%$ ). In 2011 Filipinos were less likely to have activated kp.org accounts and to have used a secure feature if they had a kp.org account (ages $25-44$ : women: $80.9 \%$ vs. $86.8 \%$ and $74.2 \%$ vs. $80.0 \%$; men: $68.2 \%$ vs. $77.6 \%$ and $60.9 \%$ vs. $63.2 \%$; ages $45-64$ : women $62.8 \%$ vs. $79.1 \%$ and $67.6 \%$ vs. $78.7 \%$; men: $58.6 \%$ vs. $75.8 \%$ and $69.1 \%$ vs. $76.5 \%$; ages $65-79$ : women $42.9 \%$ vs. $66.8 \%$ and $63.0 \%$ vs. $80.6 \%$; men: $47.5 \%$ vs. $71.8 \%$ and $68.8 \%$ vs. $85.4 \%$ ).

Discussion: Using the broad category "Asian" to study/ monitor health and health care disparities is problematic, especially for comparing populations and applying results to "Asian" populations with different Asian ethnic mixes.

Keywords: health disparities, Asian health

A2-5:

Are Race/Ethnicity and Comorbid Conditions Associated With Pregnancy Intention Status?

Mary Anne Armstrong, ${ }^{1}$ Jeanne A. Darbinian, ${ }^{1}$ Debbie A. Postlethwaite $^{1}$

\section{${ }^{1}$ Kaiser Permanente Northern California}

Background/Aims: The proportion of pregnancies in the United States self-reported as unintended (49\%), among the insured and uninsured, has not improved for more than a decade, representing a major public health problem. Unintended pregnancy has been found to be associated with poor pregnancy outcomes in numerous studies. The purpose of this study was to identify risk factors for unintended pregnancy in a health care system with equal access to all that could be used to determine groups of women who would benefit from risk reduction strategies.

Methods: In this retrospective cohort study, women age $15-44$ as of the index date of 6/30/2010 who were members of an integrated health care delivery system were followed electronically through $12 / 31 / 12$ for evidence of pregnancy
$(\mathrm{N}=45,351)$. Self-reported pregnancy intention status at entry to prenatal care (intended, unintended [mistimed or unwanted]) and potential risk factors, including age, race/ ethnicity, marital status, education/income, parity, long-acting reversible contraceptive (LARC) use prior to pregnancy, and comorbidities (diabetes, hypertension, cardiovascular disease, obesity), were obtained from electronic medical records. Pregnancy intention groups were compared on all risk factors using chi-squared tests. Logistic regression models were developed to determine which factors were associated with intended pregnancy.

Results: Bivariate analyses showed that significantly more black women had unintended pregnancies $(71.9 \%$ $[2,618 / 3,641]$ vs. $32.6 \%[5,908 / 18,107]$ white, $45.7 \%$ [4,808/10,520] Hispanic, 32.8\% [3,994/12,190] Asian, p 2 [odds ratio: 0.25 vs. $0, \mathrm{P}<0.0001$ ]), and those who did not use LARC (odds ratio: 0.44 vs. LARC, $\mathrm{P}<0.0001$ ) were significantly less likely to have an intended pregnancy. Having comorbidities was not significantly related to intended pregnancy.

Discussion: Even when there is equal access to health care, there are racial and other demographic disparities in pregnancy intention. Age-appropriate, culturally sensitive unintended pregnancy reduction interventions targeting groups of women with identified risk factors should be considered.

Keywords: pregnancy intention, racial/ethnic disparities

PS1-33:

Assessment of Cultural Competence in an Ethnic Health Program: Evidence-Based Study Miyoung Cho, ${ }^{1}$ Jane H. Kim, ${ }^{1}$ Sarah Hyun, ${ }^{1}$ Chul S.
Hyun, ${ }^{2}$ Dohyun Cho $^{1}$

\section{${ }^{1}$ W Medical Strategy Group; ${ }^{2}$ World Korean Medical Organization}

Background/Aims: Language, culture and socioeconomic determinants lead to disparities in health care. As our society becomes more ethnically and racially diverse, there is an urgent need for a health care access model, which can provide sociocultural and linguistic needs to all ethnic groups. The purpose of this study was to evaluate key factors of culturally competent health care access programs designed to address cultural and linguistic needs of underserved ethnic groups. This study utilizes a "Korean Medical Program" (KMP), an ethnic health program of a local medical center in Bergen County, NJ, as a health care access model to evaluate its program as determined by members of the community. These data are further analyzed to determine specific barriers to health access and to speculate on the elements required for cultural competence.

Methods: We utilized document analysis, surveys and interviews to evaluate experiences of a regional Korean American community on overall services provided by KMP and the local medical center. Using data from comprehensive analysis, we found sociodemographic characteristics, outcomes of health services, barriers to health care access and factors governing the effectiveness of KMP. 
Results: Data from 375 Korean American participants indicated that $51.2 \%$ were aware of KMP, and $35.7 \%$ knew about KMP health campaigns, but only $7.1 \%$ participated in campaigns. Most effective means of marketing were through television, radio and newspapers. $71 \%$ were satisfied with KMP's services. 74\% $(n=33)$ of outpatients were satisfied with the services, while only $39 \%(n=50)$ of inpatients felt the same. The profiles on access barriers depended on the ages of the participants. While the most significant barrier in the younger group (age 20-59) was cost, the most significant barrier in the older group (age 59+) was language followed by cost.

Discussion: A culturally competent health program requires numerous factors. Communication between patients and health professionals is essential, and well-trained health care interpreters are needed. Certification programs to train staff to be culturally competent are also recommended. Finally, any culturally sensitive program should engage with the community to collaborate with its health professionals to address health and welfare needs.

Keywords: ethnic health care access, Korean American

\section{PS1-34:}

\section{Disparities in Glycemic Control Among Hispanic Adults With Diabetes}

\section{David M. Mosen, ${ }^{1}$ Adrianne Feldstein, ${ }^{1}$ Ashley Borin ${ }^{1}$}

\section{${ }^{1}$ Kaiser Permanente Northwest}

Background/Aims: Poor glycemic control is associated with increased morbidity and mortality for adults with diabetes mellitus (DM). Little research has examined disparities in glycemic control among Hispanics with DM compared to whites. The objective of this work was to determine: 1) whether disparities in glycemic control exist among Hispanics versus whites; and 2) whether demographics, socioeconomic status, disease characteristics, health care utilization (primary care, specialty care, care management services) and treatment characteristics (oral hypoglycemic medications, insulin use) explain differences in glycemic control.

Methods: Using an observational study design, we studied 29,825 adults on the Kaiser Permanente Northwest DM registry as of January 1,2013 , with a valid $\mathrm{HbA} 1 \mathrm{c}$ test during calendar year 2013. Good glycemic control was defined as HbA1c 30 (vs. body mass index < 30), Charlson comorbidity score (continuous), primary care utilization in 2013 (1+ visits vs. none), specialty care utilization (1+ visits vs. none), use of DM care management services (1+ services vs. none), use of oral hypoglycemic medications $(1+$ medications fills vs. none) and insulin use (any insulin use vs. none). Seven logistic models were constructed: model 1 (race/ethnicity), model 2 (model $1+$ demographics), model 3 (model $2+$ SES), model 4 (model $3+$ disease characteristics), model 5 (model $4+$ health care utilization) and model 6 (model $5+$ treatment characteristics).

Results: Hispanics were less likely to have good glycemic control in unadjusted models (odds ratio: $0.56,95 \%$ confidence interval $[\mathrm{CI}]$ : $0.51-0.61 ; \mathrm{P}<0.0001)$. This point estimate remained consisted across all logistic models examined, even after adjusting for covariate measures (odds ratio: 0.69, 95\% CI: 0.62-0.77; $\mathrm{P}<0.0001)$.

Discussion: Our findings suggest that disparities in glycemic control among Hispanics compared to whites remain even after adjusting for critical covariate measures. More work is needed to understand whether lifestyle choices and other factors explain differences and whether targeted interventions can reduce these disparities.

Keywords: disparities, glycemic control

PS2-22:

Embedding Patients, Providers, and Community Stakeholders in Research to Improve Transgender Health

Virginia P. Quinn, ${ }^{1}$ Tracy A. Becerra,,${ }^{1}$ Theresa Gillespie,${ }^{2}$ Enid Hunkeler, ${ }^{3}$ Tisha Baird, ${ }^{1}$ Nancy M. Baisch, ${ }^{1}$ Ashli Owen-Smith, ${ }^{4}$ Douglas Roblin, ${ }^{4}$ Robert Stephenson, ${ }^{2}$ Vin Tangpricha, ${ }^{2}$ Cadence Valentine, ${ }^{5}$ Michael Goodman ${ }^{2}$

${ }^{1}$ Kaiser Permanente Southern California; ${ }^{2} E m o r y$ University; ${ }^{3}$ Kaiser Permanente Northern California; ${ }^{4}$ Kaiser Permanente Southeast; ${ }^{5}$ California State University Northridge

Background/Aims: The Institute of Medicine in its 2011 report highlights unique health challenges facing the transgender community. Evaluations of outcomes for medical gender reassignment (more appropriately termed "gender confirmation") are rare and of low quality. The literature on treatment-related quality of life is limited and little is known about the long-term effects of contra-sex hormones on the risk of age-related chronic conditions. The Study of Transition, Outcomes \& Gender (STRONG) research program will provide critical information about morbidity and mortality following gender confirmation treatments. It will also assess the comparative effectiveness of gender confirmation therapies for improving quality of life and alleviating gender dysphoria. The multidisciplinary STRONG research team includes investigators from five institutions. Members of the transgender community and health professionals who care for transgender individuals are an integral part of the team and are directly involved in all aspects of this research program including refinement of research questions, development of methods and content for data collection, and evaluation and dissemination of results.

Methods: We identified the population of transgender individuals enrolled in the Veterans Administration and Kaiser Permanente health plans in Georgia and Northern and Southern California with a combination of validated ICD codes and keyword string searches. To evaluate mortality and morbidity, we will conduct an electronic medical recordbased retrospective cohort study. To assess quality of life and other patient reported outcomes, we will conduct an online cross-sectional survey. Online and in-person focus groups with transgender stakeholders will guide data collection and interpretation of results.

Results: ICD codes and keywords identified nearly 15,000 
individuals. These preliminary findings indicate the STRONG program will represent the largest cohort of transgender individuals to date and the first such research effort in the United States. Six in-person focus groups have been completed with six more in progress. The study survey has been developed, programmed and pretested via online focus groups.

Discussion: The STRONG research program will help close the health disparities gap for the transgender community through increasing scientific knowledge. The research findings will provide guidance to clinicians and policy makers in the care they provide to this sizeable, but underserved, community.

Keywords: transgender, health disparities

\section{HEALTH INFORMATICS/VIRTUAL DATA WAREHOUSE}

\section{C2-1: \\ Measuring the Rise of Mobile and Online Care: Promises and Challenges in Big Data}

\section{Luesa L. Jordan, ${ }^{1}$ Eva Chang, ${ }^{1}$ Glen Kriekenbeck, ${ }^{1}$ James D. Ralston ${ }^{1}$ \\ ${ }^{1}$ Group Health Cooperative}

Background/Aims: Engaging patients and families is a key aspect of meaningful use of electronic medical records. Although several populations are less likely to adopt and use patient websites to engage in shared electronic medical records, the increasing availability of mobile devices and applications has potential to extend patient engagement in its use. No single data source, however, currently tracks individual patient shared record use across devices and software applications. Our objective is to describe our experience aggregating data sources for measuring enrollee use of the shared electronic medical record across mobile and desktop devices.

Methods: Between January 2010 and August 2014, we merged data from 358,415 Group Health enrollees from web server activity logs (including mobile application activity) with Epic/Clarity electronic medical record data. Web server logs were scanned for string combinations to identify devices used to access shared medical record (SMR) services (including medication refills, medical test results, secure messaging encounters, after-visit summaries, appointment requests, medical problem lists, allergies and immunizations). Logs were matched to Epic/Clarity SMR page views within a time window.

Results: In August 2014, approximately 5.5 million of 150 million web server log entries $(<4 \%)$ were extracted for device information. After excluding redundancies, 245,425 SMR entries were retained. Of these, 70\% (170,705) with device information was linked with Epic/Clarity patient page views. Of unmatched SMR activity, 29\% $(71,578)$ was on server logs alone and 1\% $(3,142)$ was on Epic/Clarity alone. Standard web browsers accounted for $88 \%(187,262)$ of overall web activity (76\% [187,262] desktop, 12\% [29,964] mobile device) and mobile applications accounted for $8 \%$ $(18,904) ; 4 \%(9,295)$ was unable to be linked. Among 94,303 enrollees accessing the SMR with device information, 92\% $(83,081)$ used standard web browsers $(78 \%[70,484]$ desktop, $14 \%[12,597]$ mobile device) and $21 \%(18,965)$ used mobile applications.

Discussion: Web server logs and Epic/Clarity can be combined to describe enrollee use of mobile and desktop services. Development and maintenance of data requires regular monitoring for consistency and content. Extraction logic must be dynamic to accommodate device market and health care system changes. Understanding adoption and use of online services across devices will be essential to successfully engaging patients and families in care.

Keywords: web server logs, shared medical record

\section{C2-3:}

\section{Categorizing Patient-Provider Secure Email Communications to Measure Substitutionary Effect on In-Person Services}

Ted E. Palen, ${ }^{1}$ Di Meng, ${ }^{2}$ Terhilda Garrido ${ }^{2}$

\section{${ }^{1}$ Kaiser Permanente Colorado; ${ }^{2}$ Kaiser Permanente Northern California}

Background/Aims: Previous observational studies have reported conflicting results when evaluating patients' use of secure email and their use of in-person clinical services. Different studies have found that secure email between patients and health care providers may substitute for, increase, or have no impact on the need for in-person clinical services. In this study we used diagnosis codes and order codes associated with emails to categorize the type of email communication. We then evaluated whether particular categories of emails substituted for the need for in-person clinical services.

Methods: We used retrospective data from Kaiser Permanente Colorado members over the age of 18 who were continuously enrolled for at least 30 months in 2010-2013. We identified which emails contained diagnosis code(s) and or order code(s). We categorized emails containing diagnosis code(s) into low acuity conditions based on diagnosis-related group categories. We then determined if the patient had a subsequent in-person appointment.

Results: Health care providers added a diagnosis code to $19 \%$ of secure emails, and $85 \%$ of these members did not have a follow-up visit or telephone call in the next 30 days. Health care providers placed a coded order within a secure email encounter $21 \%$ of the time, and $85 \%$ of these members did not have a follow-up visit or telephone call in the next 30 days. Only $8 \%$ of secure emails for low acuity conditions resulted in a follow-up visit within the next 30 days. This is comparable to rate of follow-up office visits for low acuity conditions.

Discussion: Secure email communication between patients and health care providers may substitute for the need for in-person clinical services for specific circumstances and conditions.

Keywords: secure email, patient-provider communication 
D2-1:

\section{ICD-10: A Transition That's Already Happening}

Alan E. Bauck, ${ }^{1}$ Michael Allison, ${ }^{1}$ Donald Bachman, ${ }^{1}$ Catherine Cleveland, ${ }^{1}$ Phillip Crawford, ${ }^{1}$ Suzanne Gillespie, ${ }^{1}$ Weiming $\mathrm{Hu}^{1}$

\section{${ }^{1}$ Kaiser Permanente Northwest}

Background/Aims: The United States currently uses ICD version 9 (ICD-9) for diagnosis and procedure coding. Federal regulations require that U.S. health care providers adopt the next version (ICD-10) by 10/1/2015. The new version expands the number of diagnosis codes from about 13,000 to 68,000 , and increases the number of procedure codes from approximately 4,000 to 90,000 . The Centers for Medicare \& Medicaid Services delayed its ICD-10 implementation date from $10 / 1 / 2014$ to $10 / 1 / 2015$ as a result of congressional action. However, changes to the electronic health records based on ICD-10 have continued to move forward. These changes include changing the pick lists that providers choose from as well as revamping the underlying data structures to support diagnoses coding.

Methods: The Center for Health Research Kaiser Northwest ICD-10 remediation team worked with research project staff to develop a set of diagnosis groupings (e.g. diabetes, renal disease, dementia, etc.) that can be coded under both ICD-9 and ICD-10. The team mapped the expanded provider selections to both ICD-9 and ICD-10. The team then developed a set of monitoring reports for these diagnosis groupings in order to look for points of discontinuity between ICD-9 and ICD-10.

Results: We found variation in rates of diagnosis groupings before and after the change to provider pick lists. Most diagnoses groupings have not shown a substantial change from the transition to more granularly defined diagnosis pick lists. However, when bilateral diagnosis codes are used, there can be irregularities in diagnosis rates.

Discussion: Preparing for ICD-10 has generated multiple changes to the electronic health record systems and their underlying data structures. Research projects should explore whether these changes are already impacting diagnoses and procedure-coding rates. In particular, research projects should look at trends in diagnoses rates when bilateral diagnosis codes are used.

Keywords: ICD-10, electronic health record

PS1-20:

Sustaining Use of a Clinical Decision Support Tool for Primary Care Providers

A. L. Crain, ${ }^{1}$ JoAnn M. Sperl-Hillen, ${ }^{1}$ Heidi L. Ekstrom, ${ }^{1}$ Patrick J. O'Connor, ${ }^{1}$ Karen L. Margolis, ${ }^{1}$ William A. Rush, ${ }^{1}$ Gerald H. Amundson, ${ }^{1}$ Deepika Appana ${ }^{1}$

\section{${ }^{1}$ HealthPartners}

Background/Aims: Achieving and maintaining high rates of use of clinical decision support in primary care settings has been challenging. Our goal was to reach and maintain high use rates throughout the study period through ongoing feedback and incentives to clinics and consented providers. Methods: We conducted a clinic randomized trial of an electronic health record (EHR)-based point-of-care clinical decision support (CDS) tool (called CV Wizard) that provides prioritized treatment recommendations to optimize management of six reversible cardiovascular (CV) risk factors: lipids, blood pressure, glucose, tobacco use, aspirin use and weight. We assessed use (the number of times the tool was opened and printed) at targeted office visits for two groups of primary care providers (PCPs) at 11 intervention clinics: (a) those who provided informed consent to use and evaluate the tool $(\mathrm{n}=54)$, and (b) those who did not provide consent but still had access to the CDS ( $n=69)$. CDS use rate per provider was calculated in three postintervention months as the number of eligible visits at which the tool was used relative to the number of targeted outpatient visits that month. The use goal was $80 \%$ of targeted visits, and we reported monthly use rates to clinic leaders for all PCPs, with clinic compensation totaling $\$ 2,000$ over the intervention period to achieve and maintain the goal. Generalized linear models tested whether PCP consent predicted use of the CDS system. Results: Among consented PCPs, average CDS use rates at 4, 8 and 12 months after full intervention implementation were $57.0 \%, 73.9 \%$ and $75 \%$. Among PCPs at the same intervention clinics who did not provide consent, average use rates were $57.3 \%, 70.7 \%$, and $58.9 \%$ (significant difference only at 12 months, $\mathrm{P}<0.05$ ).

Discussion: We observed robust use of the CDS tool by PCPs and rooming nurses at targeted primary care visits, in the context of targeted use to high CV risk patients only, leadership support and PCP design input, implementation process measurement and feedback, and small financial incentives to clinics that achieved high use rates. Additional evaluation to explain why use rates declined at 12 months in the nonconsented PCPs is of interest.

Keywords: clinical decision support, cardiovascular risk

PS1-35:

Expectation vs. Reality: Drug Coverage and Pharmacy Fill Rates at Group Health

\section{Roy Pardee ${ }^{1}$ \\ ${ }^{1}$ Group Health Cooperative}

Background/Aims: It seems elementary that data capture of pharmacy fills at a health maintenance organization (HMO) should be suspect for members without drug coverage. Coverage of prescriptions is a major incentive for filling prescriptions at the HMO pharmacy. Even better - even if there are scripts filled at outside pharmacies, they should generally result in the filing of a claim, which also generates fill data. Imagine our surprise, then, when it was observed that over a period of some years, Group Health members without drug coverage had significantly higher rates of pharmacy fills than did members who had drug coverage. The proposed presentation describes the investigation into this seeming anomaly.

Methods: After independently verifying that we do indeed 
observe higher fill rates in uncovered patients over a period of four years, we proceeded from the theory that the result was indication of a virtual data warehouse data problem. Either we incorrectly computed the DRUGCOV field in enrollment data, or we somehow mishandled the fills data. To investigate problems with the fills data, we recalculated rates of 30-day fills using native Group Health pharmacy data from our enterprise data warehouse. For the drug coverage flag, we isolated the set of people whose rates were anomalous, characterized their plans and coverages, and verified their drug coverage status.

Results: To our great surprise, we could readily reproduce the finding in native Group Health pharmacy data. Similarly, we found no defect in the drug coverage information. Notwithstanding this, the investigation did arrive at a plausible (if surprising) explanation for the results.

Discussion: As the saying goes, "the map is not the territory." While our intuitions and expectations about our data are necessary and powerful assets for focusing our uses of data to pursue research, it is always good to investigate and verify that our assumptions are true, and doubly important to investigate and document findings that defy conventional wisdom.

Keywords: data capture, coverage

\section{PS1-43:}

\section{Moving to a Weekly Refresh for the Virtual Data Warehouse}

\section{Alan E. Bauck, ${ }^{1}$ Catherine Cleveland, ${ }^{1}$ Shannon Leitch, ${ }^{1}$ John Brandes, ${ }^{1}$ Brenda Ackerson ${ }^{1}$}

\section{${ }^{1}$ Kaiser Permanente Northwest}

Background/Aims: Increasingly, conducting high-quality research requires access to current data. Previously, supplying current data was expensive because it required custom data extracts from source systems. In addition, these custom extracts were less robust because of frequent changes in health systems' data structures, and they could only be validated within the context of a single project. Thus, information gleaned from them was rarely fed back to the wider research institution and was not informing more general knowledge of health-system data. To address these missed opportunities, and to maximize ways the virtual data warehouse (VDW) can support research, Kaiser Permanente Northwest's Center for Health Research (CHR-NW) investigated how to change the VDW refresh rate from monthly to weekly.

Methods: We used a variety of data-management tools to change our processes and infrastructure in order to regularly deliver reliable data weekly, including: 1) We use SmartBatch to schedule weekly SAS jobs and SQL Server Agent to schedule SQL Server Integration Services (SSIS) jobs. 2) We moved to incremental builds of many data files; instead of executing a complete pull every week, we only extract data back to the point in time that it stops changing. 3) We no longer maintain a mirror copy of the VDW on an older operating system because of the cost/benefit of this task. 4) To help our transformation programs run more efficiently, we developed SSIS packages that pull source data to a local server. 5) We improved and automated our monitoring processes. 6) We established tolerance tests for when to accept or postpone data from source systems.

Results: We have been able to maintain the CHR-NW VDW on a weekly refresh cycle since June 2014, with no missed refreshes. The extract, transform and load programs that refresh our VDW run 4.4 times faster than they did just months before the transition. Our monitoring programs allow us to provide information back to our parent health system about source data.

Discussion: Changing our data systems to achieve a weekly refresh cycle was less burdensome than we first anticipated in terms of staff time. The added monitoring allows us to provide more stable data to our researchers.

Keywords: virtual data warehouse, weekly refresh

\section{PS2-27: \\ Record Linkage With Washington State Cancer Registry}

Arvind Ramaprasan ${ }^{1}$

\section{${ }^{1}$ Group Health Cooperative}

Background/Aims: At Group Health, the virtual data warehouse's (VDW) tumor registry table consists of tumor cases ascertained by Cancer Surveillance System (CSS) at the Fred Hutchinson Cancer Research Center (FHCRC). This data is limited to residents of 13 Puget Sound counties in the SEER region only. On the other hand, Washington State Cancer Registry (WSCR) consists of tumor cases for the entire Washington State residents. The poster describes the data linkage protocol and the results of linkage of Group Health members with WSCR database in the absence of unique identifier.

Methods: Group Health data consists of 1,420,334 members residing in Washington State. We linked Group Health data with WSCR database consisting of 613,631 cancer cases diagnosed during January 1, 1993-December 31, 2011. We used a probabilistic linkage software application such as CDC's Registry Plus Link Plus ${ }^{\circledR}$ to link patient information such social security number, date of birth, gender, and last and first names with corresponding data elements in WSCR. We assessed the quality of the linkage results by comparing the linkage outcome with VDW's tumor registry. We calculated the sensitivity and positive predictive value by comparing the WSCR linkage results with VDW's tumor registry. We used Venn diagrams for visualization of results of comparison.

Results: Sensitivity and positive predictive value are $90 \%$ and $96 \%$, respectively. We found 35,166 (59\%) additional tumor cases as a result of WSCR linkage in comparison to the VDW tumor registry. Of the above, 21,600 (61\%) tumor cases were reported within SEER region and 13,566 (39\%) cases diagnosed in members residing outside of Puget Sound region. Of 21,600 SEER cases, 17,248 (88\%) tumor cases were diagnosed after disenrollment from Group Health, and 2,090 SEER cases were found missing from VDW's tumor registry. Due to lack of interpretable information, we were unsuccessful in linking 5,752 records in the VDW tumor 
registry with WSCR data.

Discussion: In the absence of unique identifier, the project demonstrates a successful linkage using Link Plus. We now have cancer incidence and survival data for all Group Health members, not limited to Puget Sound region. This will augment cancer studies involving members from both SEER region and outside of Puget Sound counties.

Keywords: linkage, cancer

\section{PS2-28:}

\section{Evaluation of Provider Experience With an Electronic Health Record-Based Clinical Decision Support Tool}

Heidi L. Ekstrom, ${ }^{1}$ Patrick J. O'Connor, ${ }^{1}$ Karen L. Margolis, ${ }^{1}$ William A. Rush, ${ }^{1}$ Gerald H. Amundson, ${ }^{1}$ Deepika Appana, ${ }^{1}$ A. L. Crain, ${ }^{1}$ JoAnn M. Sperl-Hillen ${ }^{1}$

\section{${ }^{1}$ HealthPartners}

Background/Aims: Our goal was to evaluate provider experience with an electronic health record (EHR)-based clinical decision support (CDS) tool called CV Wizard implemented as part of a large randomized trial with $80 \%$ use rates for eligible patients. The tool included a quantitative "provider" form with prioritized treatment suggestions and a simpler visual companion "patient" form to efficiently elicit treatment preferences.

Methods: Two focus groups were held outside of clinic hours with a meal and $\$ 250$ compensation. Twelve providers participated and were asked to comment on open-ended questions including a) what goes into their decision to use the tool, b) the implementation process, c) how patients reacted to it, d) how it could be improved, and e) how effective it was. The discussions were audio-taped and transcribed verbatim and examined by the study team to identify themes.

Results: Providers were enthusiastic about the tool and found it valuable. They were happy that the nurse printed it for them before visits, and commented that it helped set the visit agenda and organized cardiovascular $(\mathrm{CV})$ risk information. They were more likely to discuss CV risk with patients, and indicated that they took additional time to use it with patients. There was general consensus that it was time well spent. They said the tool reinforced their treatment suggestions. Variability was noted with how nurses and providers were using the tools. For conversations with patients, some providers preferred to use the provider form over the patient form, and vice versa. The patient form was intended to be given to the patient while waiting to be seen by the provider, but this was often not happening. Providers had several suggestions for improving the use process, and asked for better documentation tools for results (smart phrases).

Discussion: A clinical decision support tool designed to help providers and patients engage in shared decision-making for $\mathrm{CV}$ risk reduction was well received and perceived as time well spent with patients. Overcoming some problems associated with workflow and adding easier ways to document use of the tool for patient discussions would add to the existing value.

Keywords: clinical decision support, cardiovascular risk
PS2-30:

Identifying Marijuana, Nicotine and Electronic Cigarette References Within a Free-Text Data Field in the Electronic Medical Record Using SAS

Bruce F. Folck, ${ }^{1}$ Stephen C. Waring, ${ }^{2}$ Christopher D. Mack, ${ }^{3}$ Catherine Cleveland ${ }^{4}$

${ }^{1}$ Kaiser Permanente Northern California; ${ }^{2}$ Essentia Health; ${ }^{3}$ Group Health Cooperative; ${ }^{4}$ Kaiser Permanente Northwest

Background/Aims: Marijuana is becoming increasingly decriminalized and legalized across the country, with no sign of abatement. Electronic cigarettes are rapidly increasing in popularity. Nicotine is a common substance utilized in the process to reduce dependency on tobacco products. Data on these behaviors are most frequently recorded in the form of free-text comment fields, and are subject to variations in spelling, abbreviation and slang. There is a growing need to be able to identify references to these behaviors in the medical record.

Methods: Using simple SAS text-string functions, variant references can be identified. A few specific "short-hand" text strings were used to capture all variations. A "short-hand string" is a series of characters one would expect to find in a word, such as "mari" and "juan" in "marijuana." Ten such strings were identified and tested for marijuana, six for electronic cigarettes and three for nicotine. When a string was identified within a field, an attempt was made to capture the entire word in which it was embedded. A manual review of this isolated candidate text was then performed to verify the integrity of the contribution for each string.

Results: Over 370 variations and misspellings of "marijuana," not including slang and cannabinoid references; over 25 variations of "electronic cigarettes;" and over 50 variations of "nicotine" have been identified. Some oversampling is expected. For example, "juan" might identify a proper noun such as San Juan Hospital; "mari" might identify marinol, an opioid but not marijuana in a conventional sense; and "huan" identified ma huang, which is a Chinese equivalent to ephedra. However, isolating the candidate text strings reduced the volume of distinct strings requiring manual review to a manageable level.

Discussion: A library of known misspellings can be employed to identify variants, but utilizing specific short-hand strings will also identify new candidates for consideration. Overinclusiveness is expected owing to idiosyncratic text entry, but isolating the candidate text will offer manageable lists for manual review when determining inclusion or exclusion of the results. Utilizing this series of shorthand strings may obviate the need to maintain an ongoing compendium of misspellings and variation.

Keywords: marijuana, natural language processing

PS2-31:

Natural Language Processing of the Unstructured Electronic Health Record Data Using Regular Expressions and SAS Hash Objects 
Paul J. Hitz, ${ }^{1}$ Mitch Juusola, ${ }^{1}$ Stephen C. Waring, ${ }^{1}$ Irina V. Haller ${ }^{1}$

\section{${ }^{1}$ Essentia Health}

Background/Aims: Structured data in the electronic health record only tell part of the story of a patient's health. Much of the information necessary for health assessment and treatment is located in notes. This information is vital for maintaining care quality as well as for use in research. Manual abstraction of this information is costly and time consuming. The goal of this study was to develop a natural language processing (NLP) system to extract health information from provider notes.

Methods: We have implemented NLP techniques in SAS using hash objects. Our workflow utilizes Excel spreadsheets to manage NLP regular expressions keywords. Keywords for the disease identification as well as keywords for generic terms, family relationships and negation are put into separate spreadsheets and used in the process. The spreadsheets are imported into SAS and loaded into hash tables. Each note is parsed into sentences and standard SAS hash processing is used to find the token and its position in the sentence. If the disease keyword is found, the family, generic and negation hash processes are run. We then utilize each of the token types and the distance from the disease keyword to identify possible instances of the condition.

Results: In a random sample of 1,000 patients, we found 195 patients with diagnosis of diabetes (23\% with ICD-9 diagnosis code only, $5 \%$ with problem listing only, and $72 \%$ with both). NLP identified 11 additional cases without diagnosis code or problem listing mention. Overall, agreement between structured and unstructured data was substantial (kappa $=0.66$ ).

Discussion: Preliminary analysis indicates that we can identify additional disease burden in patients even for well-documented conditions like diabetes. NLP is a costeffective and efficient approach for collecting information using unstructured data. In a health care delivery setting this approach offers the potential for better identification of the patients' health care needs. It also has promising implications for health research by minimizing misclassification errors and improving case ascertainment. Additional analyses are planned for other conditions that may not be as well documented using structured data.

Keywords: natural language processing, hash tables

\section{PS2-33:}

\section{A Lightweight Text Mining Tool for Multisite Research}

\section{David J. Cronkite, ${ }^{1}$ David S. Carrell ${ }^{1}$}

\section{${ }^{1}$ Group Health Cooperative}

Background/Aims: The use of electronic medical records provides researchers and care administrators with access to increased patient information. Much of the information, however, is available only as unstructured data in clinical notes, which limits its accessibility. Various natural language processing systems seek to extract information from the clinical notes and present it as structured data, thereby increasing the data's usability for research, care administration and quality assurance. Many of the available open-source tools, however, require nontrivial installation and configuration. In addition, customizations, debugging and portability can be challenging for some users.

Methods: We developed a lightweight Python application that performs basic information extraction tasks, but that is easy to install, configure, customize and share. The application relies on a dictionary to extract content from unstructured data. The dictionary is a mapping of a set of concepts (e.g. "hypoesthesia") to words and phrases that characterize how that concept would appear in clinical text (e.g. "decreased sensation," "impaired sensation," etc.). The application converts these words and phrases to regular expressions and searches the text for matching content, attempting to detect variations in spelling and to recognize when the word or phrase is qualified by negation, uncertainty, historical reference or a reference to someone other than the patient.

Results: We tested the application using a dictionary of 792 entries on a set of 205,748 clinical notes. The application completed the information extraction task in less than two hours. We used the more comprehensive Apache Clinical Text Analysis and Knowledge Extraction System (cTAKES) as a comparison using the same dictionary and same notes. We stopped cTAKES after 8.5 days with 144,701 (68.9\%) complete.

Discussion: The application provides a quick means to access unstructured data with minimal configurations and environmental settings. Multiple sites can use the same algorithm simply by sharing the dictionary and a configuration file.

Keywords: natural language processing, information extraction

PS2-34:

PopMedNet: Collecting and Using Metadata in Distributed Research Networks

Jessica M. Malenfant, ${ }^{1}$ Melanie Davies, ${ }^{1}$ Bruce Swan, ${ }^{2}$ Jeffrey Brown ${ }^{1}$

\section{${ }^{1}$ Harvard Pilgrim Health Care; ${ }^{2}$ Lincoln Peak Partners}

Background/Aims: In 2007, the PopMedNet ${ }^{\mathrm{TM}}$ platform was developed through support from Agency for Healthcare Research and Quality to facilitate multisite distributed research. This open-source software platform has been deployed in and currently supports five large-scale distributed research networks, including the Food and Drug Administration (FDA) Mini-Sentinel, CRNnet and PCORnet, with over 100 participating sites. Continued PopMedNet software development has been supported by the FDA, National Institutes of Health, Office of the National Coordinator and Patient-Centered Outcomes Research Institute, creating a scalable and extensible informatics platform that meets the needs of multiple research networks. These PopMedNet networks identified a need for improved capture and use of network metadata to enhance network operations and collaboration. 
Methods: As networks have grown in size and complexity, it is increasingly important to improve the capture and use of institutional, data source and query metadata. Organizational metadata includes information such as the organizational descriptions (e.g. health plan, hospital) available data resources (e.g. claims, registries), local expertise, data models supported and willingness to participate in different types of research activities (e.g. clinical trials, observational, etc.). Data source metadata focuses on a specific resource and includes information such as data model, data elements and periods of data capture. Query metadata include information such as query descriptions, requester and dates. New PopMedNet functionality was implemented in 2014 that allows for the capturing and reporting of network metadata.

Results: PopMedNet profile "input screens" for organizations, data sources and DataMarts (queryable data sources) were expanded to capture standardized information about each of these domains. When available, existing metadata standards were adopted. Also, the query input screen was expanded to capture additional query metadata. The PopMedNet system was expanded to allow users to search and display the metadata. Users can now search for organizations, data resources or specific queries that meet specific criteria (based on captured metadata). Access control layers managed how much information specific users can view.

Discussion: As the adoption and use of distributed networks grow, there is an increasing need to capture network metadata to improve collaboration and enable network learning. The value of distributed networks will grow with the ability to capture and use network and query metadata.

Keywords: metadata, distributed research

\section{HEALTH SERVICES RESEARCH/ HEALTH POLICY/COSTS}

\section{A1-2: \\ Increasing Preventive Care With Expanded Medicare Coverage Under the Affordable Care Act}

Sukyung Chung, ${ }^{1}$ Lenard Lesser, ${ }^{1}$ Diane Lauderdale, ${ }^{2}$ Nicole E. Johns, ${ }^{1}$ Latha Palaniappan, ${ }^{3}$ Harold S. Luft ${ }^{1}$

\section{${ }^{1}$ Palo Alto Medical Foundation Research Institute; 'University of Chicago; ${ }^{3}$ Stanford University School of Medicine}

Background/Aims: Under the Patient Protection and Affordable Care Act (ACA), Medicare coverage was expanded in 2011 to fully cover comprehensive annual preventive care visits. We assessed the impact of this coverage expansion on the utilization of preventive services.

Methods: We used 2007-2013 electronic health record data from primary care patients of Medicare-eligible age (65-75) at a large multispecialty organization in California $(\mathrm{N}=204,388$ patient-years). Using a difference-in-differences approach, we compared trends in preventive visits and recommended preventive care services across insurance coverage groups (Medicare fee-for-service [FFS] and HMO, private FFS and HMO) pre- and post-ACA. This analytic design allows us to assess the effects of the ACA controlling for contemporaneous changes in practice.

Results: Prior to the ACA, each year $1.4 \%$ of Medicare FFS enrollees had a Medicare-covered preventive visit. This rose substantially with coverage expansion to $27.5 \%$. With expanded Medicare coverage, their use of privately covered or self-paid preventive visits decreased by two-thirds, from $16.0 \%$ pre-ACA to $5.5 \%$ post-ACA, which suggests that Medicare is partially substituting for previously privately covered or self-pay preventive care visits. Even with an overall increase in preventive visit use, rates of preventive visits in 2011-2013 among Medicare FFS beneficiaries were still 10-20\% lower than other insurance groups (Medicare HMO: $52.7 \%$, private HMO: 43.5\%, private FFS: $42.6 \%$ ). Frequency of nonpreventive office visits has been decreasing, more rapidly among Medicare FFS enrollees, during the same time period. Completion of many recommended preventive services among Medicare FFS enrollees increased, particularly in those requiring extended physician time.

Discussion: Expanded preventive visit coverage under ACA led to a marked increase in preventive visits among Medicare enrollees. Preventive visits have replaced some routine office visits. The use of preventive visits among Medicare FFS enrollees may continue to increase, as the rate of utilization is still lower than seniors with private coverage only.

Keywords: preventive visit, Medicare coverage

A1-3:

Who Is Coming to Kaiser? A Comparison of the Characteristics of Estimated Eligible to Purchase Insurance Through Colorado's Marketplace to Kaiser Permanente Colorado New Members

Jennifer Barrow, ${ }^{1}$ David J. Magid, ${ }^{1}$ Jennifer Ellis, ${ }^{1}$ Susan Shetterly $^{1}$

\section{${ }^{1}$ Kaiser Permanente Colorado}

Background/Aims: An estimated 254,000 Coloradans were eligible to purchase private insurance through Colorado's health insurance marketplace. Many of those eligible chose Kaiser Permanente Colorado (KPCO). New member characteristics could impact both operational decisions and provision of quality care. This project compares characteristics of Coloradans estimated to be eligible (estimated eligible) to purchase insurance through the marketplace, those who enrolled through the marketplace, and those who chose KPCO.

Methods: This study included data on the estimated eligible population from the Colorado Health Institute's 2013 Colorado Household Access Survey of 10,224 randomly digit dialed Coloradans. Preliminary data about Coloradans who enrolled through the marketplace were collected by the administrator, Connect for Health Colorado. KPCO data were from KPCO's administrative systems and a survey of 2,570 randomly selected KPCO new members through the marketplace.

Results: As of August 2014, 145,994 Coloradans had enrolled in one of 10 carrier's health plans available through 
the marketplace. As of August 2014, 59,645 (41\%) enrollees chose KPCO. To date there were 1,017 (40\%) respondents to the survey. When compared to the estimated eligible, Coloradans who enrolled through the marketplace were older. KPCO new members were more educated (54\% vs. $27 \%$ graduated college), less likely to be Hispanic ( $9 \%$ vs. $24 \%$ ), and appeared to have a higher household income than the estimated eligible. The KPCO new members and estimated eligible were similar in the percentage reporting having usual source of health care (51\% in both groups) and seeing a provider in the previous 12 months (39\% vs. $38 \%$ ), but fewer KPCO members reported delaying seeking care in the previous 12 months because of costs (34\% vs. $42 \%)$. KPCO enrollees had similar age distributions to all Coloradan enrollees (35\% Coloradans vs. 36\% KPCO 35-54 years old; $25 \%$ vs. $27 \% 55$ years old or older).

Discussion: New members to KPCO who enrolled through Colorado's marketplace differ from the estimated eligible, but appear to be similar to all Coloradan enrollees. New enrollee characteristics inform KPCO's operational teams how to position themselves in the marketplace and provide quality health care. Knowing who enrolled is important for future plans of all carriers and concerned state health officials.

Keywords: health reform, new members

\section{A1-4: \\ Medicaid Expansion and the Affordable Care Act: Data From the First Year of Enrollment at Kaiser Permanente Northern California}

Mark W. Lin, ${ }^{1}$ Zheng Zhu, ${ }^{1}$ Wendy Dyer, ${ }^{1}$ Julie A. Schmittdiel, ${ }^{1}$ Alyce S. Adams ${ }^{1}$

\section{${ }^{1}$ Kaiser Permanente Northern California}

Background/Aims: The Affordable Care and Patient Protection Act (ACA) aims to reduce rates of un-insurance partly through expansion of Medicaid eligibility and the establishment of insurance exchanges with income-based subsidies. While prior studies used historical enrollment data to predict the characteristics and utilization patterns of newly eligible Medicaid recipients, few studies to date have actively analyzed these new enrollees. By providing MediCal coverage and commercial insurance, Kaiser Permanente provides a unique perspective when analyzing these programs. As part of a larger ongoing study, we aim to provide early data on the characteristics and preliminary utilization patterns of new Medi-Cal enrollees after the roll-out of insurance expansion under the ACA.

Methods: This is a descriptive study including two distinct cohorts of adult (18-64) Kaiser Permanente Northern California (KPNC) members who were newly enrolled in KPNC Medicaid in 2013 and 2014, defined as no KPNC enrollment in the prior 12 months.

Results: Between 1/2014 and 6/2014, 9,795 adults enrolled in Medi-Cal while 5,322 enrolled in 2013. Compared to enrollees in 2013, Medi-Cal enrollees in 2014 were older (44\% aged 41-64 vs. 29.2\%). New enrollees were predominantly female $(58.7 \%$ female vs. $41.3 \%$ male), but less so than in
2013 (62.2\% female vs. 37.8\% male). 2014 enrollees were proportionally less likely to be white $(29.0 \%$ vs. $30.0 \%)$, black $(15.3 \%$ vs. $24.5 \%)$ or Hispanic (17.0\% vs. $27.8 \%$ ), and proportionally more likely to be Asian (10.2\% vs $9.7 \%)$. However, a larger percentage was classified as "unknown" (26.8\% vs 6.2\%). Preliminary rates of utilization (per member per month) were similar, except for outpatient visits where the mean for the post-ACA cohort was 0.98 (1.59) relative to 0.74 (0.94) for the pre-ACA cohort.

Discussion: Consistent with expectations, newly enrolled Medi-Cal beneficiaries post-ACA were older and less predominantly female. However, there also was a decrease in race/ethnicity ascertainment in the post-ACA cohort. Additional analysis will explore differences in utilization once a full year of data for the 2014 cohort has been collected. When combined with data on medical comorbidities of this population, this study will help further understand the needs of the newly insured post-Medicaid expansion.

Keywords: Medicaid expansion, Affordable Care Act

A2-2:

Race-Ethnic Differences in Kaiser Permanente Northern California Seniors'Access and Preferences for Using the Web for Health Information and HealthRelated Transactions, 2013

\section{Nancy P. Gordon, ${ }^{1}$ Mark C. Hornbrook, ${ }^{2}$ Teresa Y. Lin ${ }^{1}$ \\ ${ }^{1}$ Kaiser Permanente Northern California; ${ }^{2}$ Kaiser Permanente Northwest}

Background/Aims: Patients are being encouraged to use the Internet to obtain health information and interact with the health care system. However, surveys suggest lower use of the Internet by seniors, especially blacks, Latinos, and those aged 75+. We examined whether race-ethnic and age disparities exist in a Medicare-age population regarding readiness and preferences for using the Internet for health-related purposes. Methods: In 2013 we surveyed an age-gender stratified random sample of non-Limited English Proficient white (W), black (B), Latino (L), Filipino (F) and Chinese (C) seniors aged 65-79 who were Kaiser Permanente Northern California members for $2+$ years. The study sample was linked to 2013 kp.org utilization data (activated account, use of email, online lab views, online Rx refills). SAS Proc Surveymeans and Surveylogistic were used to analyze respondent data weighted to the study population. The final sample included 849 whites, 567 blacks, 653 Latinos, 219 Filipinos and 314 Chinese.

Results: Significant $(\mathrm{P}<0.05)$ race-ethnic differences were found in easy access to a computer $(70.7 \% \mathrm{~B}, 63.0 \% \mathrm{~L}$, $57.5 \%$ F vs. $85.3 \% \mathrm{~W}, 82.8 \% \mathrm{C})$, tablet $(16.0 \% \mathrm{~B}, 12.6 \% \mathrm{~L} \mathrm{v}$. $27.1 \% \mathrm{~W}, 28.3 \% \mathrm{C})$ and smartphone $(22.0 \% \mathrm{~L}, 19.6 \% \mathrm{~F}$ vs. $31.2 \%$ W, 32.8\% B, 26.6\% C). Blacks, Latinos and Filipinos were less likely to be able to send secure messages $(50.2 \%$ B, $51.7 \%$ L, $52.0 \% \mathrm{~F}$ vs. $74.8 \% \mathrm{~W}, 73.5 \% \mathrm{C}$ ) and to prefer to use secure messaging $(22.8 \% \mathrm{~B}, 25.5 \% \mathrm{~L}, 22.0 \% \mathrm{~F}$ vs. $51.4 \% \mathrm{~W}, 55.2 \% \mathrm{C})$, view lab results online $(16.5 \% \mathrm{~B}, 17.7 \%$ L, $12.9 \% \mathrm{~F}$ vs. $35.3 \% \mathrm{~W}, 37.8 \% \mathrm{C}$ ), order $\mathrm{Rx}$ refills online (13.9\% B, 16.5\% L, 10.8\% F vs. $34.0 \% \mathrm{~W}, 36.3 \% \mathrm{C}$ ), conduct 
video visits $(12.3 \% \mathrm{~B}, 9.5 \% \mathrm{~L}, 10.6 \% \mathrm{~F}$ vs. $19.2 \% \mathrm{~W})$, and view online videos $(17.5 \% \mathrm{~B}, 15.2 \% \mathrm{~L}, 11.6 \% \mathrm{~F}$ vs. $26.7 \%$ $\mathrm{W}, 24.3 \% \mathrm{C})$. Whites were more likely to prefer to complete health risk appraisals online $(34.7 \% \mathrm{~W}$ vs. $15.6 \% \mathrm{~B}, 16.8 \%$ L, $11.6 \% \mathrm{~F}, 24.3 \% \mathrm{C}$ ), with very low preference for in-clinic tablet or interactive voice response completion across all groups. Non-Internet users were more likely to indicate the shift to web has made it harder to communicate with doctors and obtain information.

Discussion: Race-ethnic and age-related differences in access to and preference for using the Internet for health-related purposes should be considered when planning and evaluating use of patient portals and providing health information to ensure that health care access is not decreased for seniors who cannot or prefer not to use the Internet.

Keywords: patient portal use, racial-ethnic disparities

\section{B1-1: \\ Comparison of Organizational Context Across HMORN Members}

Wayne Psek, ${ }^{1}$ Jing Hao, ${ }^{1}$ James M. Pitcavage ${ }^{1}$

${ }^{1}$ Geisinger Health System

Background/Aims: Organizational context is an important element in implementation science and comparative effectiveness research (CER). Contextual factors include practice, hospital, system and environmental factors that may influence care delivery. For multisystem research networks such as the HMORN, awareness and inclusion of contextual factors can strengthen research design and interpretation of findings. Similarly, consideration of local contextual factors can enhance dissemination and implementation of interventions across systems. To our knowledge a comparative analysis of system-level contextual factors reflecting recent market and policy changes has not been performed for HMORN members. The aim of this study is to describe differences and similarities in seven system-level contextual factors across HMORN delivery systems and discuss their implications for CER and implementation research.

Methods: We performed a review of system-level contextual factors representing major policy, market or operational changes from 2004 to 2014. Contextual factors were: 1) accountable care organization (ACO) participation; 2) patient-centered medical home (PCMH) implementation; 3) CMS-CMMI program participation; 4) market consolidation (Mergers \& Acquisitions); 5) systemwide adoption of Lean or continuous quality improvement (CQI) programs; 6) Beacon Community Grants; and 7) systemwide population health management program. A manual review of several data sources including HMORN member websites, CMS website, National Committee for Quality Assurance website and Office of the National Coordinator for Health Information technology website. Additional data was attained using a keyword search in industry journals. For each contextual factor we determined a system's level of participation, start and end dates, and program or consolidation characteristics as applicable.
Results: Preliminary results indicate a wide variation across HMORN members in adoption of new models of care and market consolidation. Four members belonged to Beacon communities (health information exchanges). While most systems participated in an ACO, the funding mechanisms varied (Medicare-shared savings or Pioneer program). Systems underwent considerable consolidation, with the largest merger increasing a HMORN member size from 12 to 43 hospitals. Further results are pending.

Discussion: System-level contextual factors differ across HMORN members. Our findings draw attention to the relevance of contextual factors and may inform interpretation of results and implementation.

Keywords: delivery system, organizational context

B1-2:

Acceptance and Adoption of Lean Redesigns Among Frontline Providers in Primary Care

Dorothy Y. Hung, ${ }^{1}$ Caroline Gray, ${ }^{1}$ Meghan C. Martinez, ${ }^{1}$ Michael I. Harrison, ${ }^{2}$ Julie A. Schmittdiel ${ }^{3}$

${ }^{1} \mathrm{Palo}$ Alto Medical Foundation Research Institute; ${ }^{2}$ Agency for Healthcare Research and Quality; ${ }^{3}$ Kaiser Permanente Northern California

Background/Aims: This study examines the implementation and scale-up of Lean methodology for enhancing value in a large ambulatory care delivery system. While evidence suggests that Lean techniques can lead to higher quality care at lower cost, its success or failure is inextricably tied to the views and activities of frontline care providers who are the daily implementers of new workflows. This study explores how frontline views and activities impacted systemwide efforts to redesign primary care.

Methods: This work stems from a collaborative, mixedmethods evaluation of a major operational initiative to implement Lean as a common platform across the organization. The current analysis was guided by a modified version of the Consolidated Framework for Implementation Research, which defines various "measures" of success when implementing process redesigns. Drawing on over 100 indepth interviews with physicians and staff conducted between January 2012 and March 2014, we sought to understand the extent to which new Lean workflows were accepted and adopted into practice, and the contextual factors that impact successful implementation.

Results: Frontline views on Lean's potential to enhance value were impacted in part by: local dynamics of each care team; perceived skill or competency of team members (namely, medical assistants and licensed vocational nurses) in taking on new roles or scopes of work; and physicians' own perceived efficiency prior to the introduction of Lean redesigns. The implementation strategy used by the organization was also critical. Physicians and staff who viewed the effort as "topdown" or "inflexible" were less likely to comply with changes. Even those who expressed positive views about Lean as an overall strategy for redesigning care, but who found the implementation process excessively top-down, were less 
likely to accept and adopt new modes of operation.

Discussion: Gaining "buy-in" from frontline providers is critical to implementing workflows that are designed to improve the delivery of health care. Understanding how clinical insiders' views inform their decision to embrace or reject changes is important for managing change, and may also be instructive for organizations attempting to implement similar, operational initiatives.

Keywords: Lean process redesign, implementation measures/ success

B1-3:

Implementing a Transitional Care Program for Older Adults at High Risk for Readmission

\section{Dorothy Y. Hung, ${ }^{1}$ Francesca Nicosia ${ }^{1}$}

\section{${ }^{1}$ Palo Alto Medical Foundation Research Institute}

Background/Aims: This study describes the implementation of a transitional care program for older adults at high risk for hospital readmission. Despite increasing efforts to reduce preventable readmissions through postdischarge care coordination, little research has been done on how to operationalize transitional care in community settings. We sought to understand how implementation efforts were impacted by factors including intervention complexity, patient beliefs about transitional care, and program fidelity with ramifications on staff roles and scope of work.

Methods: Our analysis is guided by the Agency for Healthcare Research and Quality "Care Transitions Framework" (CTF), a novel adaptation of the Consolidated Framework for Implementation Research. Seventeen in-depth interviews with program staff and leadership were conducted in January and February 2014. Interviews were recorded, transcribed verbatim and analyzed by two qualitative researchers for intercoder reliability based on emergent themes and relevant constructs from the CTF.

Results: The intervention was a complex collaboration between organizations, which served to bridge multiple settings ranging from the hospital and outpatient clinic to the patient's home and community. While this partnership allowed for comprehensive services across the care continuum, it posed unique operational challenges for program coordination and communication. Challenges included engaging hospital staff to fully understand the program's goals and scope of services, particularly when making referrals. Patients' knowledge and beliefs about transitional care services also directly impacted their choice to enroll, as many older patients have preconceived notions about receiving home-based assistance and what that signifies, despite the potential benefits of such services. Finally, fidelity to the program's original coaching model was affected by midstream modifications to patient eligibility criteria. As the program was spread to other hospital units, patients with more complex medical and social needs were enrolled, resulting in increasing shifts from health coaching to clinical case management. This, in turn, created greater need for clarity around the referral process as well as staff roles and scope of work.
Discussion: This study describes contextual factors that impacted the implementation and hospitalwide spread of a transitional care program for older adults. Lessons learned can inform future efforts to implement similar transitional care interventions that are based in the community.

Keywords: transitional care, qualitative research

C1-4:

Pragmatic and Adaptive Approaches Accelerate Recruitment of Adolescents in Asthma Intervention Trial (Puff City in the Clinic)

Christine L. Joseph, ${ }^{1}$ Dennis R. Ownby, ${ }^{2}$ Edward M. Zoratti, ${ }^{1}$ Talan Zhang, ${ }^{1}$ Christine Cole Johnson, ${ }^{1}$ Mei Lu ${ }^{1}$

${ }^{1}$ Henry Ford Health System; ${ }^{2}$ Georgia Regents University

Background/Aims: Recruitment of patients for randomized clinical trials (RCT) remains a challenge for investigators. "Puff City in the Clinic" is multisite, open-label, randomized Phase II/III trial designed to evaluate a clinic-based asthma management intervention targeting urban youth. Pragmatic and adaptive approaches were used to accelerate recruitment for this RCT.

Methods: Using our electronic medical record (EMR), study eligibility criteria were applied to our patient population in order to establish a pool of potentially eligible patients and the clinics in which they were being seen. Clinical sites seeing the bulk of patients were identified, recruited, initiated and trained. Potentially eligible patients are identified through our automated appointment scheduling process and approached for enrollment at a scheduled visit. Enrollment goals were 250 patients in Phase II and 500 for total Phase II/III enrollment. Enrollment began in the second quarter of 2013. Using only onsite clinic staff to conduct recruitment, study enrollment was below target after 12 months of recruitment (57\%; 63 patients). To improve recruitment, several strategies were implemented. Recruitment reports by clinic were generated weekly to note successes as well as missed opportunities. We made use of the automated scheduling system to better identify patients with same-day appointments. We deployed research staff to compensate for staff shortages at the clinical site. Per-patient payment and responding to feedback from the clinical site helped to maintain site engagement.

Results: Enrollment rates have increased after implementation of these strategies, with 110 patients recruited in just 9 months (total to date: 106\%; 173). Real-time monitoring of patient recruitment allowed us to optimize recruitment strategies as needed, including use of the scheduling system and EMR. Key in improving recruitment is clinical site engagement, resource provision and flexible staff support.

Discussion: Onsite recruitment of patients during routine clinical care has been feasible for the RCT of an asthma intervention. Integration of the automated scheduling system allowed the identification of missed opportunities and the need for more resources. Together, these strategies have increased patient recruitment rates.

Keywords: recruitment, randomized control trial 
C3-4:

Low Reach of Four Different Alcohol Screening Approaches in the Absence of Population-Based Screening: Results From One Large Integrated Health Care System

Gwen T. Lapham, ${ }^{1}$ Emily C. Williams,${ }^{1}$ Julie E. Richards, ${ }^{1}$ Evette J. Ludman, ${ }^{1}$ Paula Lozano, ${ }^{1}$ Ryan Caldeiro, ${ }^{1}$ Amy Lee, ${ }^{1}$ Katharine A. Bradley ${ }^{1}$

\section{${ }^{1}$ Group Health Cooperative}

Background/Aims: Population-based alcohol screening is recommended for identifying patients who may benefit from brief alcohol interventions. Although few health systems universally screen primary care patients for unhealthy alcohol use, some may offer screening at specific appointments or through online health risk assessments. However, little is known about the reach of various alcohol screening approaches in the absence of universal alcohol screening. The objective was to evaluate the proportion of primary care patients who were: 1) screened for unhealthy alcohol use, and 2) screened positive as a result of four different screening approaches.

Methods: This project included 262,616 adults enrolled in Group Health in 2012 who had at least one primary care visit. Electronic medical record documentation of Alcohol Use Disorders Identification Test - Consumption (AUDIT-C) screening results were obtained from four screening approaches: online health risk assessment; progress note documentation through a template completed at preventive visits; paper-based questionnaire completed at mental health visits; and ad-hoc screening when providers had concerns. AUDIT-C results, ranging from $0-12$ points, were positive for unhealthy alcohol use at scores of $\geq 3$ and $\geq 4$ points for women and men, respectively. The percentage of patients screened and the percentage of screened patients who were positive for unhealthy alcohol use were estimated overall and across the four screening approaches.

Results: Overall, $14 \%(36,435)$ were screened with the AUDIT-C. The approach-specific percentages of patients screened were $8 \%$ online, $3 \%$ at preventive visits, $1 \%$ at mental health visits and 3\% by ad-hoc screening. Of those screened, $33 \%$ were positive for unhealthy alcohol use and the approach-specific percentages of patients who screened positive were $35 \%$ online, $28 \%$ at preventive visits, $40 \%$ at mental health visits and $45 \%$ by ad-hoc screening.

Discussion: Despite four approaches to alcohol screening, $86 \%$ of primary care patients were not screened for unhealthy alcohol use. Formal implementation of universal screening is likely necessary to achieve high rates of alcohol screening. Keywords: unhealthy alcohol use, preventive screening

D1-1:

Primary Care Provider Perspectives on Reducing Low-Value Care

Diana S. Buist, ${ }^{1}$ Matthew R. Handley, ${ }^{1}$ Eva Chang, ${ }^{1}$ Roy Pardee, ${ }^{1}$ Gabrielle Gundersen, ${ }^{1}$ Allen Cheadle, ${ }^{1}$ Robert J. Reid ${ }^{1}$
${ }^{1}$ Group Health Cooperative

Background/Aims: Constraining costs and enhancing value in health care delivery is a national priority. Understanding the clinician perspective is particularly important because health care professionals play key roles in choosing what health care services are used. This study explores clinicians' perceived use of and professional responsibility for reducing low-value care, barriers to decreasing its use, and knowledge and perceived legitimacy of the Choosing Wisely campaign.

Methods: We conducted an online survey of 304 primary care clinicians at Group Health in Fall 2013. The overall response rate was $62 \%(\mathrm{n}=189)$.

Results: Nearly all (93\%) responded that the cost of care they personally deliver is important to different stakeholders and believe it is fair to ask providers to be cost-conscious. Over half (62\%) said patients request unnecessary tests or procedures at least several times per week. The majority indicated they were somewhat or completely comfortable discussing low-value care with colleagues $(70 \%)$ and patients $(88 \%)$; conversations with patients were reported much more frequently than with providers $(10 \%$ and $56 \%$ five or more discussions in the past 30 days, respectively). Providers indicated patients follow their advice the majority of the time about unnecessary tests or procedures when conversations happen. Notable perceived barriers to decreasing low-value care included: time (45\%), community standards (43\%), challenges overcoming patient preferences and values (43\%), fear of patients' dissatisfaction (40\%), patients' knowledge about harms of having low-value care (37\%), availability of tools to support relevant shared decision-making (36\%) and fear of litigation (31\%). Almost two-thirds of providers were aware of the national Choosing Wisely campaign, nearly all of which considered it a legitimate source of information on unnecessary tests and procedures.

Discussion: There were few differences in national physician surveys and our survey of integrated delivery system clinicians - patients listen to their providers and the majority of providers report talking with patients about reasons to avoid unnecessary tests. Awareness and perceived legitimacy of the Choosing Wisely campaign suggests the campaign and others like it may be used to activate providers to be conscientious stewards of limited health care resources. Additional focus on training providers to have discussions with colleagues about low-value care could be beneficial.

Keywords: low-value care, Choosing Wisely

D1-2:

Risk Stratification and Population Management: Validation of the Patient Stratification Model Based on Electronic Health Record

Irina V. Haller, ${ }^{1}$ Brian P. Johnson, ${ }^{1}$ Michael Van Scoy, ${ }^{1}$ Catherine VonRueden, ${ }^{1}$ Paul J. Hitz, ${ }^{1}$ Joseph Bianco ${ }^{1}$

\section{${ }^{1}$ Essentia Health}

Background/Aims: Population health management and patient risk stratification are essential components of health 
care delivery in order to achieve the Triple Aim of better health outcomes, better care and lower health care costs. Many current risk adjustment/risk stratification models are based on claims data, which often are unavailable in health care setting. This study evaluated an electronic health recordbased patient stratification model (PSM) and its accuracy in predicting future utilization of high-cost health care services.

Methods: A 24-item scoring system, PSM includes markers for chronic conditions, disease states, lab values, health behaviors and health care utilization. In this retrospective cohort study, we focused on adult (18+ years old) primary care patients from a regional health care system $(\mathrm{N}=250,903)$. Patients were classified based on demographics, place of residence (isolated rural or small rural, large rural, urban) and prevalence of PSM markers at baseline (9/1/11-8/31/12). The outcome measure in this analysis was defined as 3 or more emergency department (ED) visits at follow-up (9/1/12$8 / 31 / 13)$. We used a logistic regression model to estimate the odds ratio (OR) of the baseline factors on the outcome using both unweighted and weighted PSM scoring. Weights for the PSM markers were calculated as ratios of deviations of the OR from one and the sum of the deviations. The unweighted and weighted composite scoring systems were assessed on the basis of predictive characteristics of the models (c-statistic). In addition, we will examine interactions between age groups and PSM markers and use bootstrap methods of resampling to assess the variation in predictions.

Results: The average age in the cohort was 49.0 (standard deviation: 19.2) years at baseline, with $37.0 \%$ being 40 or younger and $23.0 \%$ being 65 or older; $54.3 \%$ were female and $57.9 \%$ resided in rural areas. Frequent ED use at followup was observed in $2.7 \%$ of the study cohort. The c-statistics for the models using unweighted and weighted baseline PSM markers were 0.834 and 0.868 , respectively, with a difference of 0.034 (95\% confidence interval: $0.032-0.037)$.

Discussion: A model that includes weighted PSM markers, demographics and place of residence had better accuracy in predicting future frequent ED use. Additional analyses will help with further refinement and calibration of the model.

Keywords: risk stratification, electronic health record

\section{D1-3:}

Linking Medication Orders to Prescription Fills: Lessons From HMO Enrollees Applicable to PPO Patients

Su-Ying Liang, ${ }^{1}$ Laura J. Eaton, ${ }^{1}$ Jia Pu, ${ }^{1}$ Tony Chen, ${ }^{1}$ Harold S. Luft ${ }^{1}$

\section{${ }^{1}$ Palo Alto Medical Foundation Research Institute}

Background/Aims: This study develops an approach to estimate the prescription fill rate when detailed medication orders from electronic health records are available but pharmacy dispensing data are limited.

Methods: Data included medication orders from the electronic health records and pharmacy claims from a large ambulatory group practice with mixed payment sources. Pharmacy claims were available only for HMO patients, but no direct link was available to track whether a pharmacy claim was tied to a particular medication order. We analyzed 608,871 medication orders from 2007 to 2013 for 26,875 HMO patients who were enrolled in an HMO plan in the calendar year preceding, during and after the medication ordering date. We developed an algorithm matching the pharmacy claims to medication orders by the patient identifier, the generic product identifier and the prescription fill date within the eligible timeframe (i.e. within 365 days of the ordering date or between the ordering date and a specified termination date). Fill rates were computed separately for the index (i.e. newly initiated therapy) and renewal orders for each drug. Sensitivity analyses were conducted using different matching algorithms, e.g. using simple generic name and medication strength.

Results: Overall, $75 \%$ of the 608,871 medication orders had matched pharmacy claims using the proposed algorithm. Fill rates were higher among renewal orders than index orders (76\% vs. $74 \%, \mathrm{P}<0.01)$. Preliminary findings suggested a higher fill rate for chronic conditions compared to acute conditions $(78 \%$ vs. $74 \%, \mathrm{P}<0.01)$. In addition, there were variations across therapeutic classes, ranging from $83 \%$ fill rate of anti-infective agents to $40 \%$ of nutritional products. Sensitivity analyses using different matching criteria yielded a similar overall fill rate $(73.6 \%)$.

Discussion: We developed a generic approach to estimate the prescription fill rates based on medication orders and pharmacy claims from HMO patients. This is an important first step, as the estimated fill rates can serve as a benchmark and be applied to patients who did not have pharmacy dispensing data in the same delivery system. Applications of estimated fill rates can be further extended to estimate pharmacy costs associated with medication orders or examine patterns of medication adherence over a range of conditions.

Keywords: prescription fill, medication orders

\section{D1-4: \\ Severe Obesity on Labor and Delivery: Variation in Hospital Resources Among California Hospitals}

Daniele S. Feldman, ${ }^{1}$ Moshe Fridman, ${ }^{2}$ Lisa Bollman, ${ }^{3}$ Lisa Korst, ${ }^{4}$ Samia El Haj Ibrahim, ${ }^{1}$ Arlene Fink, ${ }^{5}$ Kimberly D. Gregory ${ }^{1}$

${ }^{1}$ Cedars-Sinai Medical Center; ${ }^{2}$ AMF Consulting; ${ }^{3}$ Community Perinatal Network; ${ }^{4}$ Childbirth Research Associates; ${ }^{5}$ University of California Los Angeles

Background/Aims: More than half of pregnant women are overweight and $8 \%$ of reproductive aged women are extremely obese. Rates of maternal obesity and obstetrical complications associated with obesity are increasing. How hospitals are responding to this trend in terms of both clinical practices and resources is unknown. The objective of this study is to describe how labor and delivery (L\&D) units in California are equipped to care for women with extreme obesity.

Methods: A cross-sectional study based on a survey instrument designed and validated to collect information on childbirth practices and resources from nurse managers on L\&D units in all California hospitals. Based on responses 
to specific survey items, hospitals were stratified by type (Community, Teaching, and Integrated Delivery System [IDS] hospitals) and obesity resources (low level [LL] — at least one key piece of equipment not available in hospital; intermediate level [IL] — all equipment available in hospital but not kept on L\&D; and high level [HL] — all equipment available in hospital and kept on L\&D). Survey questions identified hospitals that transfer out women and ascertained the availability of the following resources to accommodate women with severe obesity: operating table, special gurney, surgical instruments and retractor equipment.

Results: Nearly all hospitals responded to the survey (239/248 [96\%]). There were 187 community hospitals, 27 teaching hospitals and 25 IDS hospitals. Of these, there were $77 \mathrm{HL}$ (32.2\%), 143 IL (51.5\%) and 39 LL (16.3\%) hospitals. Of the 25 IDS hospitals, there were $15 \mathrm{HL}(60 \%), 8 \mathrm{IL}(32 \%)$ and 2 LL ( $8 \%$ ). Only one IDS hospital reported that they had ever transferred a patient out for extreme obesity and this was a LL hospital.

Discussion: Hospitals vary by resources available to manage obese patients. IDS hospitals are better equipped to care for obese patients with nearly twice as many HL IDS hospitals than the total hospital sample. The extent to which this is associated with patient outcomes remains to be determined and is the focus of ongoing research. Primary outcomes should include cesarean delivery rates and composite maternal morbidity among obese patients who delivered at hospitals with LL, IL and HL obesity resources

Keywords: hospital resources, severe obesity

D1-5:

The Risk of Subsequent Concussion or Reconcussion Among Youth Participating in Sports

Debbie A. Postlethwaite, ${ }^{1}$ Diane Carpenter, ${ }^{1}$ Sarah Preiss-Farzanagan, ${ }^{1}$ Catherine Broomand ${ }^{1}$

\section{${ }^{1}$ Kaiser Permanente Northern California}

Background/Aims: In 2012, an amendment to the California Education Code (CA-AB 25) was enacted requiring schoolbased athletic programs to remove youth from the sport played when a concussion occurred, and to provide more rigorous evaluation and treatment. Youth were required to obtain written clearance from their health care provider before returning to play.

Methods: A retrospective cohort study was conducted of Kaiser Permanente Northern California (KPNC) members age 10-17 years with sports-related concussions from 2009-2010 and 2012-2013. Our aims were to calculate subsequent concussion (reconcussion) rates and compare risks for reconcussion before and after enactment of CA-AB 25. Data obtained determined type of sport, level of contact (noncontact, limited contact, contact and collision), presenting symptoms, and evidence of prior and reconcussion to the index concussion. Chi-square tests and logistic regression analysis were performed to assess the association of index concussion demographic (age, gender, race/ethnicity, median family income) and clinical characteristics with reconcussion.
Results: Preliminary results $(\mathrm{N}=1,002)$ of KPNC youth age 10-17 in 2009-2010 showed concussion diagnoses were highest among boys playing football (31.6\% [223/706]) soccer (8.8\% [62/706] and baseball (8.64\% [61/706]). Concussion diagnoses were highest among girls playing soccer $(27.7 \%$ [82/296]), softball (10.47\% [31/296]) and basketball (10.14\% [30/296]). Reconcussions occurred in $7.4 \%$ within 18 months. Reconcussions among those with concussions prior to index compared with those without were higher (11.6\% [22/189] vs. $6.5 \%$ [52/795], $\mathrm{P}=0.02)$. Those playing contact/collision sports were more likely to have reconcussions compared with limited/noncontact sports (9.4\% [53/561] vs. $5.0 \%$ [21/417], $\mathrm{P}=0.01)$. Of reconcussed subjects, those who played contact/collision sports were more likely to be reconcussed due to sports participation compared with those who played limited/noncontact sports $(81.1 \%$ [43/53] vs. 52.74\% [11/21], $\mathrm{P}=0.02)$. In multivariable analyses, age 14-17 compared to age 10-13 (odds ratio [OR]: 2.59, 95\% confidence interval [CI]: 1.36-4.97), and blurred vision/light sensitivity (OR: 2.10, 95\% CI: 1.16-3.78) and dizziness or lightheadedness (OR: 1.75, 95\% CI: 1.02-2.98) vs. absence of these symptoms were significantly associated with reconcussion.

Discussion: Preliminary findings show risk of reconcussion in athletic youth is a serious public health problem. Final results on 2,065 subjects will show comparisons in reconcussion rates and risk factors associated with reconcussions before and after enactment of CA-AB 25.

Keywords: sports-related concussion, reconcussion

\section{D3-2:}

Estimating the Human Resource Costs of Developing and Implementing Shared Medical Appointments in Primary Care

Cheryl D. Stults, ${ }^{1}$ Sean McClellan, ${ }^{1}$ Laura Panattoni, ${ }^{1}$ Mary Carol Mazza, ${ }^{1}$ Ming Tai-Seale ${ }^{1}$

\section{${ }^{1}$ Palo Alto Medical Foundation Research Institute}

Background/Aims: Shared medical appointments (SMAs) or group visits have been shown to improve access, but there is no research to date on the costs of developing and implementing SMAs. We estimate the human resource costs of developing and implementing an administrative program to support SMAs and an additional SMA on cancer survivorship. Methods: Activity-based costing was used to analyze the human resource costs for completed tasks, personnel involved and time to complete tasks. The study occurred in one division of a nonprofit, multispecialty group practice in northern California that serves 126,000 patients. We conducted indepth, key-informant interviews with stakeholders involved in SMAs - clinicians, coordinator, and operations manager - from different levels of the organization. National median wages were calculated from the 2013 Bureau of Labor Statistics.

Results: We estimate that the human resource cost to develop and implement a program to support SMAs was $\$ 62,874$. The employee who spent the most time (1,255 hours) was the SMA coordinator and the activity that required the most time $(1,211$ 
hours, \$52,945) was for research tasks including establishing workflow and logistics of a SMA program. Once the SMA program was established, the cancer survivorship SMA cost $\$ 7,271$ to develop and implement. The physician who ran the cancer survivorship SMA spent the most time and provided the most expensive labor (72.5 hours, \$6,513) and the most time-consuming and costly activity was the development of materials (44 hours, \$3,768).

Discussion: Developing and implementing a program to support SMAs took the SMA coordinator about 8 months. Introducing new providers or a new type of SMA may require a relatively modest incremental commitment of organizational resources and provider time (less than $6 \%$ of the time and less than $14 \%$ of the finances required to start the initial SMA). It is possible that the total time and cost could be further decreased by leveraging relevant materials from existing SMAs.

Keywords: primary care, activity-based costing

\section{ECl-2: \\ Reductions in Medical Resource Use Among Primary Care Physicians Following the Adoption of Personalized, Transparent Reporting}

Eva Chang, ${ }^{1}$ Diana S. Buist, ${ }^{1}$ Matthew R. Handley, ${ }^{1}$ Roy Pardee, ${ }^{1}$ Gabrielle Gundersen, ${ }^{1}$ Robert J. Reid ${ }^{1}$

\section{${ }^{1}$ Group Health Cooperative}

Background/Aims: With Group Health's commitment to Affordable Excellence, Group Health has implemented multifaceted interventions since 2011 around helping clinicians become aware of their health services use relative to their colleagues, with the goal of increasing quality and reducing waste. This quality improvement initiative aims to measure changes in physician medical resource use and identify physician practice characteristics associated with decreasing use of low-value services.

Methods: We calculated physician ordering rates for specialty referrals, prescriptions, high-end imaging, and lab tests for 197 practicing primary care physicians with panels greater than 500 in 2012 and 2013. Our primary outcomes were the differences between 2013 and 2012 ordering rates per 1,000 patients adjusted for age, sex and clinical complexity. Multiple regression models were used to examine associations between outcomes and physician years in practice and FTE-adjusted panel size. Models also were adjusted for gender and ethnicity. Results: In 2012, mean (with standard deviation) primary care physician ordering rates for referrals, prescriptions, imaging and labs were 336.1 (142.7), 7,838.2 (1,601.3), 19.8 (10.6) and 1,805.7 (647.5), respectively. Between 2013 and 2012, ordering decreased across all services; mean change in referrals, prescriptions, imaging and labs was -89.6 (125.7), $-1,255.5(1,509.5),-5.3(8.9)$ and -435.9 (562.1), respectively. For referral, imaging and lab test orders, reduction was greatest in physicians newer to practice. Also for lab test orders, physicians with smaller panels had greater reductions. We did not observe any significant practice characteristics associated with change in prescriptions.

Discussion: Group Health physicians with fewer years practicing and smaller panel sizes were associated with larger decreases in resource use from 2013 to 2012. The larger decrease in resource use in newer physicians may be associated with a learning curve early in practice and may represent an important time for early feedback and intervention. Since the multifaceted intervention also may have influenced physician resource use, more understanding of what accounts for reductions in resource use is useful for future development of interventions.

Keywords: resource utilization, primary care practice

PD2-5:

\section{Adverse Effects of Fecal-Based Colorectal Cancer Screening}

Grace Clarke Hillyer, ${ }^{1}$ Christopher D. Jensen, ${ }^{2}$ Wei K. Zhao, ${ }^{2}$ Alfred I. Neugut, ${ }^{1}$ Benjamin Lebwohl, ${ }^{1}$ Douglas A. Corley ${ }^{2}$

\section{${ }^{1}$ Columbia University; ${ }^{2}$ Kaiser Permanente Northern California}

Background/Aims: Individuals report that the colorectal cancer (CRC) screening process is stressful and, for some, studies indicate that this stress has a downstream impact on motivation to engage in health behaviors and use of health care services. We examined ambulatory primary care (APC) utilization following a false-positive fecal immunochemical test (FIT) to screen for CRC among patients enrolled at the Kaiser Permanente Northern California PROSPR site between 2007 and 2011.

Methods: Included were adults aged 50-89 years who were enrolled in Kaiser Permanente Northern California continuously for 36 months. The first FIT completed during this period for those with a true-negative FIT (negative FIT and not diagnosed with CRC in the subsequent 12 months) and a false-positive FIT (no CRC or adenoma detected with diagnostic colonoscopy following a positive FIT) was identified. The mean number of APC visits in the 12 months following the FIT screening result was compared to the prior 12-month period. Associations between differences in APC utilization pre- to post-FIT by test result and patient demographic characteristics and Charlson comorbidity score were assessed.

Results: We examined 582,997 electronic medical records. For true-negative FIT, we did not detect a change in the number of APC visits pre- vs. post-FIT, whereas there was an increase among patients with a false-positive FIT. Comparison of mean APC visits pre- to post-FIT screening for true-negative FIT was -0.02 (standard deviation [SD]: 2.4] vs. 0.57 [SD: 2.6] for false-positive FIT $(\mathrm{P}<0.001)$. In multivariable analysis, those with a false-positive FIT were $64 \%$ more likely to have increased APC utilization post-FIT compared to those with a true-negative FIT and that utilization tended to increase with age. Those with no comorbidities were $45 \%$ more likely to increase their APC utilization post-false-positive FIT, and this association decreased as comorbidities increased. Men were less likely than females to engage in increased APC utilization post-false-positive FIT. 
Discussion: This study demonstrates that primary care utilization varies significantly by fecal-based colorectal cancer screening test result. Further evaluation of the nature of these visits, as well as underlying behavioral determinants of the utilization is warranted.

Keywords: fecal-based colorectal cancer screening, harms of cancer screening

PS1-2:

Using Systematic Review and Decision Modeling to Fill Gaps in Evidence: Application to Positron Emission Tomography Scan Use in Posttreatment Squamous Cell Carcinoma of the Oropharynx

Susan R. Snyder, ${ }^{1}$ Steven S. Chang, ${ }^{2}$ Jing Hao, ${ }^{1}$ James M. Pitcavage, ${ }^{1}$ Kevin Wang, ${ }^{3}$ Scott A. Kono, ${ }^{4}$ Daniel M. Saman, ${ }^{5}$ Thuy-Anh Melvin, ${ }^{6}$ Gabriel G. Calzada ${ }^{7}$

${ }^{1}$ Geisinger Health System; ${ }^{2}$ Henry Ford Health System; ${ }^{3}$ Kaiser Permanente Northern California; ${ }^{4}$ Kaiser Permanente Colorado; ${ }^{5}$ Essentia Health; ${ }^{6}$ Mid-Atlantic Permanente Research Institute; ${ }^{7}$ Kaiser Permanente Southern California

Background/Aims: There is a general lack of costeffectiveness evidence for positron emission tomography (PET) scan use in cancer suitable to support decision-making, and specifically for post-nonsurgical treatment of squamous cell carcinoma of the oropharynx (SCOOP). The value of effectiveness research is greatest when there are considerable data challenges and uncertainty due to early adoption of new technology and/or emerging serious high-growth disease. New expensive technology commonly becomes the standard of care without adequate evidence to support its value, including whether its performance does more good than harm to patients compared to the previous standard or alternatives. An example is the lack of evidence-informed standards for postchemoradiation imaging of SCOOP for patient management. The standard of care is posttreatment physical examination with imaging at 8-12 weeks using PET scan, computed tomography scan or ultrasound. Due to limits in the sensitivity and negative predictive value of these alternatives, patients may be over- or undertreated. A particular concern is PET scans are routinely used in practice although they have a high false-positive rate, which often result in unnecessary procedures.

Methods: A recommended efficient and evidence-based approach to providing needed information addressing health care cost and quality research gaps is decision and simulation modeling informed by a systematic review of the literature to obtain relevant information for model assumptions and parameter values augmented by expert input and consensus review. This approach will be applied to three posttreatment imaging alternatives for human papillomavirus (HPV)positive SCOOP patients, and supplemented by Cancer Research Network (CRN) virtual data warehouse (VDW) patient data from HMORN participants and a technical expert panel from the newly formed CRN Head and Neck Cancer Scientific Interest Group.
Results: The results presented are a decision-analysis model with assumptions, parameters and input values documented from the systematic review, CRN VDW data and input provided by the CRN Head and Neck Cancer Scientific Interest Group.

Discussion: The finalized decision-analysis model will support simulations to estimate comparative effectiveness outcomes and cost-effectiveness of the HPV-positive SCOOP post-nonsurgical treatment imaging alternatives to provide much needed evidence to evaluate and improve clinical decision-making and patient outcomes and to identify future research needs.

Keywords: systematic review, cancer

PS1-4:

Variation in Pathology Examination of Extended Core Prostate Biopsies: A CRN Pilot Project

Mara M. Epstein, ${ }^{1}$ Christopher L. Owens, ${ }^{2}$ Daniel Peterson, ${ }^{3}$ Vanessa Neergheen, ${ }^{3}$ Valerie McManus, ${ }^{4}$ Deborah Multerer, ${ }^{4}$ Robert T. Greenlee ${ }^{4}$

${ }^{1}$ University of Massachusetts Medical School; ${ }^{2}$ University of Massachusetts Memorial Medical Center; ${ }^{3}$ Meyers Primary Care Institute; ${ }^{4}$ Marshfield Clinic Research Foundation

Background/Aims: Extended core prostate biopsy (ECPB) removing 10-12 tissue cores is the standard of care in men investigated for prostate cancer; however, the manner of pathologic examination varies. Urologists may process cores by laterality into two specimen jars, or separate each core into 12 specimens for pathologic review. Considering the high volume of ECPBs performed annually, varying strategies of submitting specimens for review could lead to variations of over $\$ 1$ billion in health care expenditures. We will examine the association between number of jars submitted for pathologic review and rates of prostate cancer detection and equivocal diagnoses.

Methods: We are conducting a pilot project at two Cancer Research Network (CRN) sites - Meyers Primary Care Institute (MPCI) in Worcester, MA, and Marshfield Clinic (MCRF) in Marshfield, WI - to determine patterns of processing ECPB tissue cores for pathologic review in men aged 30-95 years who underwent ECPB between 2008-2011. ECPBs and subsequent pathology services were identified in the CRN virtual data warehouse (VDW). Prostate cancer diagnoses were identified in VDW tumor files and equivocal diagnoses as repeat ECPBs within 7-180 days of the initial biopsy. Site-specific methods for identifying and validating jar number have been developed.

Results: To establish this pilot study, we constructed datasets using the CRN VDW, discovered new variables in raw claims data, and performed medical record abstraction to validate new variables. At MPCI, 731 ECPBs were conducted 20082011, with a median of 2 jars per ECPB (range: 1-36). Jar number was extracted from raw claims data and validated through selected medical chart review. At MCRF, 902 ECPBs were conducted 2008-2011, with a median of 3 jars per ECPB 
(range: 1-12). A jar number algorithm that partly includes manual abstraction for some patients was developed and validated through a systematic review of all identified biopsy patients. Additional analyses linking jar number to patient outcomes are ongoing.

Discussion: A two-site CRN pilot project was successfully developed to investigate the association between jar number for ECPB pathologic review and patient prostate cancer outcomes. Combining VDW and raw claims data, we have established a strong foundation for future research in this area. Keywords: prostate biopsy, pathology

PS1-14:

Cost-Effectiveness of Taxanes in the Treatment of Metastatic Breast Cancer: A Review of the Literature

\section{Erika L. Estrada, ${ }^{1}$ Albert J. Farias, ${ }^{1}$ Janet Mora Marquez, ${ }^{1}$ Cecilia Portugal, ${ }^{1}$ Aniket A. Kawatkar ${ }^{1}$}

\section{${ }^{1}$ Kaiser Permanente Southern California}

Background/Aims: Metastatic breast cancer (MBC) is an incurable disease in which regardless of treatment, total survival time is often less than a year. In these patients suffering from $\mathrm{MBC}$, mitigation of symptoms, reducing side effects of therapy and enhancing quality of life are the major issues in selecting a treatment. Taxanes have been shown to be efficacious as single agents or in combination with other agents as compared to other chemotherapy regimens. Docetaxel has received more support over paclitaxel in many analyses that have compared the two drugs indirectly. A direct comparison of both docetaxel and paclitaxel has not been possible until recently due to a lack of randomized trials. Little is known in reference to cost-effectiveness of taxanes in the treatment of $\mathrm{MBC}$ when compared directly. The objective of this literature review was to assess cost-effectiveness literature comparing docetaxel and paclitaxel in MBC.

Methods: PubMed was used to conduct a search for manuscripts published 2005-2014. Search terms included "metastatic breast cancer" in addition to "cost-effectiveness" $(n=96)$, “cost-utility" ( $n=9)$, “docetaxel" $(n=332)$ and "paclitaxel" $(\mathrm{n}=336)$. Only articles that included costeffectiveness analyses and a direct comparison among the taxanes were included.

Results: A total of three cost-effectiveness studies were identified and included in the final review. Two of the three studies utilized data from a randomized controlled trial. The cost-effectiveness analyses reported incremental cost-effectiveness ratios (ICER) and quality adjusted lifeyears (QALY) and concluded that docetaxel, compared to paclitaxel, was superior and improved QALYs by $0.33-0.75$. It is important to note that all three analyses were conducted outside of the United States.

Discussion: In North America, breast cancer remains the most frequently diagnosed cancer in women. Yet, there are no new comprehensive economic analyses comparing the two taxanes published recently in the United States. Literature reflecting such comparisons has been published within the United Kingdom, Spain and Canada (reflected in this review).
However, with the prevalence for the disease growing, it is important to continuously develop models assessing costeffectiveness among patients with MBC.

Keywords: cost-effectiveness, metastatic breast cancer

PS1-22:

Inability of Primary Care Providers to Predict Medication Fulfillment of New Prescriptions

Eric A. Wright, ${ }^{1}$ Ryan P. VanLoan, ${ }^{1}$ Agnes S. Sundaresan, ${ }^{1}$ J. B. Jones, ${ }^{2}$ Jing Xie, ${ }^{3}$ Michael A. Fischer ${ }^{3}$

${ }^{1}$ Geisinger Health System; ${ }^{2}$ Sutter Health Research; ${ }^{3}$ Brigham and Women's Hospital

Background/Aims: Physician prediction of patient medication adherence to chronic therapy is unreliable, but the accuracy of physician predictions is largely unstudied for new prescriptions. Our aim was to determine if provider perception of the likelihood a patient will pick up a medication is an accurate predictor of primary medication nonadherence.

Methods: We conducted a prospective cohort study as part of a randomized clinical trial. Providers at 24 primary care and family medicine Geisinger clinics were asked to complete a "best practice alert" (BPA) within the electronic health record when placing an order for a new antihypertensive, antidiabetic, antihyperlipidemic or antiasthmatic medication. The BPA asked: "In your opinion, how likely is it that this patient will pick up this medication?" The provider could select from a 5-level Likert item with responses ranging from "very unlikely" to "very likely." Provider response was correlated to the principle outcome variable, medication first fill after 14 days as identified from the records of the pharmacy to which the prescription was transmitted.

Results: A total of 4,822 patients over 11 months were included, and 4,532 (94\%) patients filled their prescription within 14 days. Providers answered the BPA $89 \%$ of the time. Among respondents, most felt their patients would be likely or very likely to pick up their new medication $(90.6 \%$ vs. $86.8 \%$ of providers chose likely or very likely among adherent and nonadherent groups, respectively). Only 10 (3.9\%) of new medication orders not filled (nonadherent) versus 110 (2.7\%) filled (adherent) were suspected by providers to be unlikely or very unlikely to be picked up, resulting in only an $8.3 \%$ positive predictive value for primary medication nonadherence.

Discussion: Our study suggests that physicians overwhelmingly believe their patients are likely/very likely to pick up their first prescription. A physician's intuition about a patient's likelihood of filling a new medication does not reliably identify patients who do not fill new prescriptions for chronic medications. Our study's ability to assess prediction accurately is limited by the unusually high first-fill rate of patients in this trial. Our findings are congruent with other reports assessing physician perceptions of patient adherence to chronic medications.

Keywords: primary adherence, prediction 
PS1-36:

\section{Consultation and Referral Patterns of Primary Care} Nurse Practitioners

\section{Michelle L. Cook, ${ }^{1}$ Lydia Riley, ${ }^{1}$ Tyler Litsch ${ }^{1}$}

\section{${ }^{1}$ American Association of Nurse Practitioners}

Background/Aims: The aims of the Primary Care Nurse Practitioner Consultation \& Referral Patterns study included: a) determining the percentage of patients for which primary care nurse practitioners (NPs) request referrals or formal consultations; and b) exploring the patient, provider and system characteristics associated with variation in consultation/referral. Specifically, the project will contribute to understanding the decision-making models for when and how patients are referred by NPs to physicians and other health care providers.

Methods: Primary care NPs who were individual members of the American Association of Nurse Practitioners were invited to participate in the study. During a 3-week period, participants recorded summary data identifying: a) all the patients seen on a particular day, b) the number of patients seen by age category, and c) the type of patient visit. When any visit occurs in which they initiate an informal consult, formal consult or referral, the NPs recorded de-identified data to describe the encounter, preferably at the time they completed their routine charting after each of these encounters.

Results: Results are forthcoming. Data collection is ongoing through September 30, 2014, and the analysis will be completed by February 2015. Descriptive statistics will be used to examine the distribution of key outcome and predictor variables. Means and standard deviations will be computed for continuous variables and percentages for categorical variables. These data will be used to describe the study participants, address the research questions and inform the statistical analysis.

Discussion: Conclusions will contribute to understanding the decision-making models for when and how primary care NPs are consulting and referring.

Keywords: nurse practitioners, consultations, referrals

PS1-37:

Evaluation of an Advanced Patient-Centered Medical Home Model at Kaiser Permanente Los Angeles Medical Center

William N. Grice, ${ }^{1}$ Anthony L. Morena, ${ }^{1}$ Richard A. Mehlman, ${ }^{1}$ Zakiya N. Devine, ${ }^{1}$ Huong Q. Nguyen, ${ }^{1}$ Ernest Shen, ${ }^{1}$ Angelika Clarke $^{1}$

\section{${ }^{1}$ Kaiser Permanente Southern California}

Background/Aims: Organizing cost-effective patientcentered care for individuals with complex medical and social needs continues to be challenging for many health care systems. The patient-centered medical home (PCMH) is a popular model that emphasizes enhanced access to teambased comprehensive and coordinated care that actively engages patients and their families in self-care and that which is supported by appropriate tools and technologies to facilitate proactive population-based management. Operationalizing these core principles into sustainable practice beyond mere attainment of national recognition is still the subject of intensive experimentation across many health systems, including Kaiser Permanente Southern California (KPSC). The aim of this study is to evaluate the effects of an enhanced PCMH care model at one medical center in KPSC.

Methods: We will use a retrospective propensity-score matched control design to compare changes in the primary composite outcome of hospitalizations and emergency department visits in the 12 months before and after AdvancedPCMH implementation. The care transformation included changes to the structures, functions, roles/responsibilities of the care team, workflows and processes of care; chief among these changes was formalizing the role of a care navigator and embedding social medicine resources and behavioral health within primary care. Advanced-PCMH was implemented in two primary care modules with 14 providers. A total of 300 patients who were high utilizers of hospital-based services in the previous six months or at increased risk for use of hospitalbased services due to their utilization patterns in the previous six months and patients who were 90 years of age or older were enrolled. Approximately 600 patients will be selected from five comparable primary care modules to serve as controls. We also will measure level of exposure to Advanced-PCMH care elements, standard quality-of-care processes, behavioral and clinical outcomes, patient satisfaction, quality of end-oflife care, and perceptions of team functioning, satisfaction and level of burnout by providers.

Results: We are in the process of extracting the data and will have results available at the meeting in March 2015.

Discussion: Findings from this evaluation will inform future efforts in our system to achieve the triple aim of better care and outcomes at potentially lower cost.

Keywords: patient-centered medical home, evaluation

\section{MENTAL HEALTH/CHEMICAL DEPENDENCY}

C3-1:

Smoking Prevalence and Use of Cessation Medications Among Patients With Psychiatric Disorders in an Integrated Health Care Delivery System: A Case-Control Study

Kelly C. Young-Wolff, ${ }^{1}$ Andrea H. Kline-Simon, ${ }^{1}$ Constance Weisner ${ }^{1}$

\section{${ }^{1}$ Kaiser Permanente Northern California}

Background/Aims: Although impressive gains have been made in recent decades in reducing overall rates of smoking in the United States, individuals with behavioral health conditions continue to smoke at high rates and have limited success with quitting. The current study tested whether longitudinal disparities in smoking prevalence would also be found among individuals with behavioral health conditions in an integrated health care delivery system with convenient 
access to tobacco treatments.

Methods: This secondary analysis examined smoking prevalence over four years in a subsample of Kaiser Permanente Northern California patients diagnosed with the five most prevalent behavioral health conditions (cases) in $2010(\mathrm{n}=155,733)$ : depression, anxiety, substance use disorders, bipolar spectrum disorders, and attention deficit hyperactivity disorders (ADHD). Nonbehavioral health condition-matched controls were created for all unique cases $(n=155,733)$. Smoking status for each of the behavioral health cases and matched controls was compared over a 4-year period (2010-2013) using a logistic regression generalized estimating equation model. Logistic regression analyses also were used to compare tobacco cessation medication use in 2010 among smokers with behavioral health conditions and smoking-matched controls.

Results: Participants with behavioral health conditions had significantly higher odds of smoking over the 4-year period compared to matched controls $(\mathrm{P}<0.001$ for all). Patients with substance use disorders and bipolar spectrum disorders had the largest differences in odds of smoking relative to their matched controls. Smoking prevalence decreased over 4 years overall, but to a lesser extent among cases with depression, anxiety, substance use disorders and bipolar disorder compared to controls $(\mathrm{P}<0.001$ for all). With the exception of ADHD, smokers with behavioral health conditions were significantly more likely than matched controls who smoked to utilize tobacco cessation medications in 2010 ( $\mathrm{P}<0.001$ for all).

Discussion: Similar to findings in the general U.S. population, results from an integrated health care delivery system indicate that smoking prevalence is decreasing at slower rates among individuals with versus without behavioral health conditions. Findings highlight the potential to address smoking among disproportionately impacted groups within a health care setting.

Keywords: behavioral health conditions, smoking prevalence

\section{C3-2:}

Removing Barriers to Preventive Care for People With Serious Mental IIInesses: Preliminary Results From the PRIME Study

Bobbi Jo H. Yarborough, ${ }^{1}$ Scott Stumbo, ${ }^{1}$ Micah T. Yarborough, ${ }^{1}$ Carla A. Green ${ }^{1}$

\section{${ }^{1}$ Kaiser Permanente Northwest}

Background/Aims: People with serious mental illnesses (SMI) experience premature morbidity and mortality, largely due to preventable conditions, and may not receive guidelineconcordant preventive care. Understanding modifiable factors that produce health disparities among this population will inform efforts to improve care delivery and reduce excess mortality.

Methods: Interview and survey data was obtained from primary care providers (PCPs) practicing in a not-for-profit integrated health plan, Federally Qualified Health Centers and safety-net clinics, and patients with SMI. Formative interviews with both groups explored approaches, experiences, barriers and perceived effectiveness of preventive services for people with SMI. Web-based surveys with PCPs $(n=249$; response rate $=51 \%$ ) and interviews with patients diagnosed with schizophrenia-spectrum disorders, affective psychosis or mood disorders, followed formative interviews. Patient data collection is ongoing (projected $\mathrm{n}=150$ ).

Results: PCPs felt patients with SMI lacked future orientation and interest in prevention and perceived that these patients have too many needs and are seen too infrequently to affect their health. For providers, lack of time was the most common barrier to providing preventive care $(89 \%)$. Providers felt office visits were too short, particularly when patients were experiencing psychiatric symptoms; 59\% said psychiatric stability greatly/moderately affected ability to provide preventive services. Patients agreed visits were short but saw symptoms as a barrier only when they interfered with making/ attending appointments or made providers uncomfortable. Provider-patient relationships were a barrier if providers were dismissive/rude. Patients mentioned appointment logistics (e.g. scheduling, transportation), costs, avoidance and forgetfulness as barriers. A lack of interest in prevention was not supported among patients. Both groups mentioned lack of time, lack of coordination with mental health providers, lack of insurance coverage and lack of understanding insurance as barriers.

Discussion: To increase preventive service use among patients with SMI, patients need assistance overcoming logistical barriers that prevent them from getting into the office. Psychiatric symptoms can make it difficult to get in, but once patients are in front of the PCP they are amenable to prevention, particularly when recommendations come from a provider who engages them. Providers need more time, resources that support collaboration with mental health providers, and some may need additional training to increase their comfort with these patients and their confidence that their recommendations can be effective.

Keywords: preventive services, serious mental illness

C3-3:

Diabetes Mellitus and Rheumatoid Arthritis Predict Depression Disorder Diagnosis in Nonelderly Adults in Primary Care Settings

Euijung Ryu, ${ }^{1}$ Alanna M. Chamberlain, ${ }^{1}$ Richard S. Pendegraft, ${ }^{1}$ William V. Bobo, ${ }^{1}$ Jyotishman Pathak ${ }^{1}$

\section{${ }^{1}$ Mayo Clinic}

Background/Aims: Depressive disorders (DEP) are often comorbid with other chronic conditions. Bidirectional associations between DEP and these conditions are poorly understood. In this study, we aimed to identify chronic conditions with the greatest influence on the DEP diagnosis.

Methods: A validated electronic health record-based algorithm was applied to identify DEP patients receiving primary care at Mayo Clinic between 2000 and 2013. Cases were identified on the basis of having $>2$ DEP-related ICD9 diagnosis codes, $>1$ antidepressant prescription, and $>3$ 
mentions of in- or outpatient clinical notes for DEP. Controls were matched on birth year ( \pm 2 years), sex and outpatient clinic visits at the same year. We ascertained 26 chronic condition categories, as defined by Chronic Conditions Data Warehouse, using the 5 years of medical records prior to the DEP diagnosis. For each age group at diagnosis (60 years), gradient boosting machine models were applied to estimate relative influence $(\mathrm{RI})$ of the chronic conditions on the DEP diagnosis.

Results: A total of 11,219 DEP cases were identified (median age at DEP diagnosis 44 years, $34 \%$ male and $89 \%$ white). The proportion of subjects with at least one co-occurring condition increased with older age and was higher in DEP cases compared to controls ( $9.5 \%$ vs. $6.9 \%$ for subjects 60 years). For subjects aged $\leq 45$ years, diabetes mellitus ( $\mathrm{RI}=19.6 \%$ ) was identified as the most influential condition contributing to the risk of DEP, followed by asthma ( $\mathrm{RI}=11.6 \%$ ) and rheumatoid arthritis $(\mathrm{RI}=11.0 \%)$. For subjects aged $46-60$ years, the most influential condition was rheumatoid arthritis $(\mathrm{RI}=13.2 \%)$ followed by diabetes mellitus ( $\mathrm{RI}=12.9 \%)$. For subjects older than 60 years, dementia $(\mathrm{RI}=10.7 \%)$ and rheumatoid arthritis $(\mathrm{RI}=10.2 \%)$ showed similar relative contribution to the risk of DEP.

Discussion: Among nonelderly adults who received primary care at Mayo Clinic (2000-2013), diabetes mellitus and rheumatoid arthritis were the strongest risk predictors for a diagnosis of depression among 26 chronic conditions considered. Our results suggest that certain chronic conditions may play a role in exacerbating, precipitating or increasing longitudinal risk of medically significant depression.

Keywords: depression, electronic health record

\section{C3-5: \\ Single-Item Screens for Prescription and Illicit Drug Misuse Largely Identifies Primary Care Patients With Unrecognized Drug Use}

\author{
Katharine A. Bradley, ${ }^{1}$ Gwen T. Lapham, ${ }^{1}$ Ryan Caldeiro, ${ }^{1}$ \\ Larry Marx ${ }^{1}$
}

\section{${ }^{1}$ Group Health Cooperative}

Background/Aims: The U.S. Preventive Services Task Force recommends depression and alcohol screening as part of routine primary care, but not drug screening because brief primary care counseling about drug use does not improved outcomes. However, drug screening might be helpful if it led to identification and treatment of patients with unrecognized substance use disorders. The objective was to describe the prevalence of positive drug and marijuana screens in a primary care population and whether patients who screened positive had previously recognized substance use disorders.

Methods: We conducted a 3-week feasibility test of universal depression and alcohol misuse screening in one primary care clinic as a prelude to behavioral health integration. Providers asked that we also screen for illicit drug and marijuana use (the latter was legal in Washington state). A total of 409 $(61 \%)$ of 667 patients who were seen in the primary care clinic for a 3-week period completed a one-page behavioral health questionnaire that included brief depression (PHQ-2), alcohol misuse (AUDIT-C), drug (validated single-item) and marijuana screens. The latter was adapted from the singleitem drug screen.

Results: Of 409 patients who completed screening $(68 \%$ women; $10 \%, 17 \%, 43 \%$ and $31 \%$ age $<30,30-44,45-64$ and $\geq 65$ years, respectively), $4 \%$ screened positive for past-year prescription or illicit drug use, $13 \%$ reported marijuana pastyear use (3\% daily or almost) and $16 \%$ screened positive for alcohol misuse. Among the 16 patients who screened positive for drug misuse, 4 (25\%) reported daily drug misuse, and 7 (44\%) screened positive for alcohol misuse. Only $1(6 \%)$ of the 16 patients who screened positive for drug misuse had a prior diagnosis of a substance use disorders (but not in the past year). However, 38\% had been prescribed opioids and $13 \%$ benzodiazepines in the past year (38\% either). Providers found drug screening valuable as they had been previously unaware of most patients' drug use.

Discussion: Most patients who screened positive for illicit or prescription drug misuse did not have recognized substance use disorders. Findings suggest that routine screening with single-item drug and marijuana screens provide important clinical information for primary care teams.

Keywords: drug misuse, preventive screening

\section{PS1-32:}

The Utilization of the Search Function of the Scoring Website for the Handbook of Normative Data for Neuropsychological Assessment for Research of Underserved Populations

Katherine W. Smith, ${ }^{1}$ Ariella Benji, ${ }^{1}$ Nardos Bellete, ${ }^{1}$ Payam Kharazi, ${ }^{1}$ Hussah Al-Kharafi, ${ }^{1}$ Rodger Questin, ${ }^{1}$ Marcia Nimmer, ${ }^{1}$ Paula Cedillo, ${ }^{1}$ Enrique Lopez ${ }^{1}$

\section{${ }^{1}$ Cedars-Sinai Medical Center}

Background/Aims: Clinicians and neuroscience researchers are still faced with the cumbersome task of searching through handbooks, journals and online databases to search for neuropsychological norms when assessing individuals on neuropsychological measures, specifically those from diverse populations.

Methods: In order to make the acquisition of neuropsychological normative data more simple and efficient, and to increase the validity of appropriate neuropsychological measures for specific populations, the Handbook of Normative Data for Neuropsychological Assessment (Mitrushina et al., 2005) has now been computerized and is easily accessible online. A specific search function (http://npnorms.com/ search) in the computerized database allows a clinician to input demographic information (e.g. age, education, gender identity, country of origin and language) to determine what norms are available for some of the most common neuropsychological measures used (e.g. Trail Making Test, Color Trails Test, Stroop, Grooved Pegboard and Wisconsin Card Sorting Test).

Results: This newly developed, Internet-based program allows the examiner to fill in demographic information to find 
the appropriate measures to administer to a specific client. Additionally, this Internet tool can be utilized for research purposes in determining where important normative data is lacking for at-risk individuals.

Discussion: Since all information is consolidated in one location and consistently updated to reflect available research on culturally appropriate measures and normative data, this is particularly useful and efficient for neuroscientists who are both conducting research and are providing clinical care, particularly those who see underserved minority groups.

Keywords: neuroscience, cognition

\section{PATIENT-CENTERED OUTCOMES RESEARCH/PATIENT AND STAKEHOLDER ENGAGEMENT}

D4-1:

\section{Best Practices in Patient Engagement in Research: Creating an Engagement Checklist}

\section{Sarah D. Madrid, ${ }^{1}$ Leslie A. Wright ${ }^{1}$}

\section{${ }^{1}$ Kaiser Permanente Colorado}

Background/Aims: With the establishment of the PatientCentered Outcomes Research Institute in 2010 and subsequent explosion in recognition of the importance of patient perspectives in research to improve patient decisionmaking and health outcomes, patient engagement in research is an increasingly critical aspect of the research process. The HMORN Patient Engagement in Research Scientific Interest Group (PER SIG) was established to improve the ability of member organization research teams to effectively and meaningfully engage patients and other stakeholders in the continuum of research, from developing appropriate research questions to disseminating research findings. One of the 2014 goals for the PER SIG was to create an engagement checklist to guide research teams in planning and recruiting for patient engagement, and provide best practices for their engagement. Methods: We created a comprehensive questionnaire encompassing all aspects of the engagement process, including prompts for intentionality/justification for engaging patients, patient roles and stages of participation, logistical details and planning, human resource issues (e.g. compensation, contracts), IRB oversight, recruiting, training for patient and research team members, communication plans, and engagement monitoring and evaluation. We fielded our web-based survey to all members of the PER SIG and other research colleagues with experience engaging patients as members of research teams.

Results: We received 23 responses to the survey. From the results and comments provided, we created a draft checklist of items and practices critical to the engagement process. This draft was vetted by PER SIG members and discussed at a monthly PER SIG conference call, then finalized as a "working draft." Broader dissemination is currently underway, and this abstract, leading to further dissemination at the annual meeting, is one goal of the dissemination plan.

Discussion: Patient engagement in research is a complex process requiring thoughtful planning and preparation as well as continued monitoring and evaluation throughout the life of the research project. The patient engagement checklist provides research teams with guidance for each step of the process, promoting use of best practices and encouraging meaningful engagement of patients as members of the research team.

Keywords: patient engagement, patient-centered outcomes research

\section{D4-2:}

Building Infrastructure to Engage Patients, Families and Members in Research Within a Learning Health Organization

\section{Jane E. Duncan ${ }^{1}$}

\section{${ }^{1}$ HealthPartners}

Background/Aims: HealthPartners is the largest consumergoverned nonprofit health care organization in the country, with a mission "to improve health and well-being in partnership with our members, patients and community." We strive to have members, patients and families (PFM) experience us as a trusted partner, and have engaged over 30 advisory groups across the enterprise. The HealthPartners Institute for Education and Research is an integral arm of the HealthPartners learning health organization. One of the institute's key goals is to develop strategy, processes and tools to engage PFM in all aspects of patient-centered outcomes research (PCOR).

Methods: Institute leadership prioritized the development of an infrastructure to support PFM engagement in research. A stakeholder group of three senior investigators and the Strategic Programs Manager led the effort to align with the larger HealthPartners strategy. Other resources included HMORN's patient engagement scientific interest group, the Institute for Patient- and Family-Centered Care, the Patient-Centered Outcomes Research Institute, and the Park Nicollet Medical Group, which had recently merged with HealthPartners. The HealthPartners compliance, legal and finance teams advised on appropriate security and privacy training and financial issues. The IRB provided guidance on appropriate review and approval. Investigator team leaders were interviewed to identify priorities and gaps. Finally, a discussion was held with a patient advisory group currently serving on a PCORI-funded grant led by Dr. Leif Solberg.

Results: By joining the HealthPartners core PFM strategy team, the institute has access to a full inventory of PFM partnerships across the enterprise. A defined process and toolkit were created to recruit, interview and onboard PFM partners. Formal job descriptions and contract templates are available. A compliance guide that defines privacy and security training has been created and approved. Training for investigators and project managers will be held to share tools and best practices. The process will be piloted in an upcoming PCORI submission.

Discussion: Engaging PFM at all levels of the HealthPartners organization, including Research, is one of our core values. 
By designing tools and practices that have been vetted and easily adapted, we hope to streamline the process of involving these valuable partners in research.

Keywords: patient engagement, best practices

\section{D4-3: \\ Developing a Patient-Facing Report for Delivering Results After Whole Genome Sequencing}

Alanna Kulchak Rahm, ${ }^{1}$ Janet L. Williams, ${ }^{1}$ Audrey L. Fan, ${ }^{1}$ Heather L. Stuckey, ${ }^{2}$ Doris T. Zallen, ${ }^{3}$ Jamie Green, ${ }^{1}$ Michele Bonhag, ${ }^{1}$ Lynn Feldman, ${ }^{4}$ Michael Segal, ${ }^{4}$ Marc S. Williams ${ }^{1}$

${ }^{1}$ Geisinger Health System; ${ }^{2}$ Penn State Hershey College of Medicine; ${ }^{3}$ Virginia Tech; ${ }^{4}$ SimulConsult Inc.

Background/Aims: Genome sequencing is emerging into clinical practice, raising a number of issues for delivery systems. One question is how genomic results can be made available wherever the patient receives care. The purpose of this research is to develop a patient-facing genomic laboratory report with advanced functionality including point-of-care education and clinical decision support. Report development utilized providers and parents of affected patients to provide feedback on the desired report elements to maximize usability.

Methods: The research team, including a patient investigator and experts in patient engagement and communication, developed a draft report. Study participants were parents of children with undiagnosed cognitive disability undergoing genome sequencing as part of a larger clinical research study. Semistructured interviews were conducted to elicit prior experience with genetic test results and feedback about the draft report. Based on feedback, subsequent focus groups were conducted to elicit comments on specific report elements presenting inheritance and prognostic information. Recordings of interviews and focus groups were transcribed and analyzed using the conceptual framework of existential phenomenology, which favors the interpretation of meaning through subjective experiences.

Results: Participants endorsed the importance of having a report created for patients and family. In particular, they noted desire to read and re-read the report and to have a record of what was discussed. The draft report was found to be informative and written at an appropriate level. Focus groups reviewed and ranked four different options for presenting prognostic information. Important themes for this specific information were seeing how the condition may change over time and information on support and additional action steps to take.

Discussion: Participants value a report created for them. The interview and focus group participants are informing the creation of a report that will be used in the comparative effectiveness portion of the larger project to provide actual results of whole genome sequencing.

Keywords: patient engagement, genome sequencing
D4-4:

Partnering With Patient Advisors in the PORTAL Clinical Data Research Network

Carmit K. McMullen, ${ }^{1}$ Maureen H. Rumptz, ${ }^{1}$ Ameerah Thomas, ${ }^{2}$ Julie N. Harris, ${ }^{2}$ Stephanie R. Prausnitz, ${ }^{2}$ Carol P. Somkin²

\section{${ }^{1}$ Kaiser Permanente Northwest; ${ }^{2}$ Kaiser Permanente Northern California}

Background/Aims: PORTAL, composed of Kaiser Permanente, Group Health Cooperative, Health Partners and Denver Health, is a PCORnet network focusing start-up efforts on identifying what matters most for patients with obesity, colorectal cancer and congenital heart disease (three cohorts) and building capacity to conduct patient-centered research to address those priorities. This presentation will describe the Patient Engagement Council (PEC) and an associated Online Advisor group guiding PORTAL's development.

Methods: The PEC is composed of 10 patients representing each of the cohorts as well as three representatives from cohort-specific advocacy organizations. PEC members attend an in-person meeting for orientation/team-building and subsequently offer guidance through 10 webinars, emails and conference calls. PORTAL seeks PEC members' input about how to engage patients in research, identify research questions and methods that matter to patients, and facilitate communication between PORTAL and broader audiences. Eighteen online advisors are being recruited to help PORTAL establish three cohort-specific online Smart Patients communities - where patients and caregivers learn from each other about the latest science, treatments and patient concerns. Online advisors work with Smart Patients to identify key topics and develop early content for the online communities' discussion boards.

Results: Eligible members were identified within each health system through clinical and operational liaisons and were then interviewed by PORTAL staff. Selected candidates were invited to join as PEC members or online advisors. All of the PEC members have been recruited, completed the orientation, and participated in the first webinar focused on cohort-specific survey questions/modes of survey administration. The successes and challenges of recruitment, hosting in-person and online meetings, and PEC contributions to PORTAL's start-up phase will be presented. Prior to the conference, all 18 online advisors will have been recruited and completed their work setting up online communities. The benefits and challenges of engaging online advisors will be presented and the online communities will be described.

Discussion: This presentation of the process and initial outcomes of engaging patients in the development of a large multisite network will have broad significance as a model for engaging patients in research network development.

Keywords: patient engagement, patient-centered outcomes research 
D4-5:

\section{Engaging Patients as Stakeholders to Foster More} Open Communication With Primary Care Providers

\author{
Cheryl D. Stults, ${ }^{1}$ Ellis C. Dillon, ${ }^{1}$ Amy Meehan, ${ }^{1}$ Judith
} Chuang, ${ }^{1}$ Ming Tai-Seale ${ }^{1}$

\section{${ }^{1}$ Palo Alto Medical Foundation Research Institute}

Background/Aims: The philosophy of the Patient Centered Outcomes Research Institute (PCORI) is to "do research differently" by involving patients throughout the research process. By leveraging an existing resource of a senior "aging in place" community, we recruited patients to be stakeholders and help develop an intervention to create more open communication between primary care providers and patients. This was one of our initial forays into engaging patients as stakeholders. We wish to share our experience on the patient engagement journey.

Methods: A reflexive, qualitative summary of our observations on the process of engaging patient stakeholders during all phases of a research study.

Results: Five patient stakeholders, all older retired individuals, participated through all stages of the research process, attending regular stakeholder meetings and events and responding to impromptu requests by email or phone. Our patient stakeholders were an important part of the construction of the study materials, a visit companion booklet and a postvisit survey, and we featured their pictures and comments on the back of these tools so that study participants could better relate to their experience. One stakeholder was a former marketing professional who was instrumental in helping us formulate and sharpen the language and delivery of the intervention. When we encountered study recruitment problems, we held a patient stakeholder meeting to seek their advice. They provided us with suggestions that we incorporated into our workflow.

Discussion: Expanding the relationship between patients and researchers was easier than expected and yielded a great improvement on the design and conduct of this research study. Engaging patient stakeholders was not only invaluable for the researchers but also created a reciprocal relationship. The researchers were asked by patient stakeholders for guidance in their own projects, e.g. in one case a patient asked the research team to help her plan an advance care planning workshop at a senior center. Our older retired patient stakeholders are not representative of all patients, but their availability allowed them to share their cumulative wisdom and insights - not only as patients, but also as resourceful professionals - with the research team.

Keywords: patient engagement, patient-centered outcomes research

\section{PS1-15:}

A Systematic Review of the Measurement Properties of the European Organization for Research and Treatment of Cancer QLQ-BR23 Quality-of-Life Instrument
Janet Mora Marquez, ${ }^{1}$ Albert J. Farias,${ }^{1}$ Cecilia Portugal, ${ }^{1}$ Erika L. Estrada, ${ }^{1}$ Aniket A. Kawatkar ${ }^{1}$

\section{${ }^{1}$ Kaiser Permanente Southern California}

Background/Aims: The objective of this literature review was to analyze the measurement properties of the European Organization for Research and Treatment of Cancer's (EORTC) breast cancer-specific quality-of-life questionnaire (QLQ-BR23).

Methods: A PubMed search was conducted using the search terms "breast neoplasm," "quality of life," and "EORTC" to identify all the articles using the EORTC QLQ-BR23 questionnaire. The search was limited to studies published in the English language from 2005 to 2014. Articles were also searched on HEALTHSTAR and Google Scholar Search and the websites of organizations like the Centers for Disease Control and Prevention, the National Cancer Institute and the EORTC Quality-of-Life group. This study primarily focused on articles looking solely at measurement properties such as discriminative and evaluative properties, reliability, validity and interpretability of the EORTC QLQ-BR23 instrument. Articles that did not report any measure of reliability, such as intraclass correlation coefficient or Cronbach's alpha, were excluded from the review.

Results: Of the 101 articles obtained from the initial search, 18 were relevant and 15 met the inclusion criteria. The QLQ-BR23 instrument had suitable face and content validity. Of those 15 articles that met the inclusion criteria, only 6 established support for convergent-divergent validity. Acceptable convergent-divergent validity was seen in the body image, sexual functioning, systemic therapy side effects, breast symptoms and arm symptoms domains. Construct validity for all domains was supported using the method of known groups in 3 culturally diverse populations when the QLQ-BR23 was used as a discriminating tool. Overall, the instrument exhibited high internal consistency in the diverse populations. Reliability, interpretability and evaluative properties were inadequately studied in the published studies. Discussion: The EORTC QLQ-BR23 instrument is proven to be valid cross-culturally and readily accepted as having high internal consistency. Consequently, it is a suitable instrument for measuring patient-reported outcomes in breast cancer patients. It has good discriminative properties, but evaluative properties need more empirical support. Considerations for future research may include using the QLQ-BR23 module in studies designed to better assess the instrument's evaluative properties.

Keywords: EORTC QLQ-BR23, quality of life

PS1-18:

A Pharmacy Counseling and Postdischarge Intervention to Improve Initial Adherence for HighRisk Medications

John E. Zeber, ${ }^{1}$ Disha Shah, ${ }^{1}$ Angel Trieu ${ }^{1}$

${ }^{1}$ Scott \& White Health System 
Background/Aims: Poor medication adherence to potentially life-saving prescriptions frequently inhibits appropriate treatment and outcomes, particularly concerning new drugs following hospital discharge. Yet such initial adherence signals an opportunity to implement effective patient-centered care, balancing clinical objectives with patient health beliefs and other considerations. Within quality improvement efforts of a pharmacy counseling program for high-risk medications, we utilized a short adherence questionnaire and follow-up contact with patients to determine factors associated with initiating anticoagulant regimens.

Methods: Consecutive adults discharged from Scott \& White Hospital between February and December 2013 receiving new prescriptions for Plavix, Effient, Brilinta, Pradax, Xarelto or Coumadin were recruited. Per program guidelines, a pharmacist counseled inpatients concerning their prescriptions, discussed benefits and side effects, and helped resolve immediate adherence problems. Individuals were next administered a validated 3-item Likert instrument as a brief, proximal screener for adherence propensity targeting specific barriers on medication trust, perceived effectiveness and out-of-pocket drug costs. Following consent, participants were contacted within 3 days to provide demographics and confirm they filled initial prescriptions, commenced treatment and intended to remain adherent. If not, self-reported reasons for not taking medications were solicited. Descriptive and bivariate analyses documented associations between patient characteristics, perceived barriers, and upon initial adherence behavior.

Results: Slightly over half of the 101 patients contacted (96\% response rate) were prescribed Plavix, with other medications equally distributed; participants averaged 70.1 years, were $60 \%$ male, $26 \%$ minority and $12 \%$ uninsured. The mean medication trust score was 4.7 (of 6), indicating positive drug perceptions, as did the 4.8 average for gauging prescription importance. However, mixed experiences were reported for personal drug costs (3.6 mean). 94\% both filled initial prescriptions and intended to keep taking them. Minimal self-reported barriers included costs or forgetting to visit the pharmacy, yet far greater numbers enthusiastically supported the counseling program and follow-up contact.

Discussion: While the brief screening instrument did not identify patients likely to experience adherence problems (costs excluded), the predischarge counseling program exemplifies a successful transition engagement as patients initiate essential treatment. Since prior work demonstrates that highly activated individuals adopt better self-care behaviors, including adherence, such educational patientcentered interventions can promote healthier decisions and better outcomes.

Keywords: medication adherence, transition counseling

PS1-25:

Assessing Current Practices in the Evaluation and Treatment of Acute Sinusitis: A Comparison of Primary Care, Urgent Care and Emergency Department Care
Adam L. Sharp, ${ }^{1}$ Marc Klau, ${ }^{1}$ David Keschner, ${ }^{1}$ Eric Macy, ${ }^{1}$ Tania Tang, ${ }^{1}$ Ernest Shen, ${ }^{1}$ Corrine MunozPlaza, ${ }^{1}$ Michael Kanter, ${ }^{1}$ Matthew A. Silver, ${ }^{1}$ Brian Mittman, ${ }^{1}$ Michael K. Gould ${ }^{1}$

\section{${ }^{1}$ Kaiser Permanente Southern California}

Background/Aims: Acute sinusitis (AS) affects 1 in 7 adults and costs over $\$ 5.8$ billion annually. As part of the American Board of Internal Medicine's Choosing Wisely campaign, three organizations make recommendations regarding appropriate sinus imaging and antibiotics for uncomplicated AS. The extent to which AS recommendations are followed is unknown; our aim is to measure adherence with recommendations for AS and to compare AS encounters in primary care (PC), urgent care (UC) and emergency (ED) departments.

Methods: A retrospective, observational study of AS care using structured data from electronic health records, complemented by more extensive chart review in a smaller subsample of encounters. All immunocompetent adults with an initial AS encounter (ICD-9 code 461.x) from 2010 to 2012 were included. Primary outcomes (structured data) were filled antibiotic prescriptions and the performance of a face, head or sinus computerized tomography (CT) scan, and secondary outcomes (chart review) were length of symptoms and adherence to AS recommendations.

Results: The median age of patients was 46 (interquartile range: $34-58), 67.5 \%$ were female and the mean Elixhauser comorbidity score was 1.5 ( \pm 1.6 standard deviation). Of $152,774$ AS encounters (77\% PC, $22 \%$ UC and $1 \% \mathrm{ED})$, $89.2 \%$ resulted in a filled antibiotic prescription and $1.1 \%$ in a CT scan. Compared with the PC setting, UC encounters were more likely to result in antibiotics (adjusted odds ratio [OR]: $1.12,95 \%$ confidence interval [CI]: 1.08-1.17) or obtaining a CT scan (adjusted OR: 2.4, 95\% CI: 2.1-2.7, and ED encounters were less likely to result in antibiotics (adjusted OR: $0.57,95 \%$ CI: $0.50-0.65$ ) but more likely to result in a CT scan (adjusted OR: 59.4, 95\% CI: 51.3-68.7). Chart review of 300 encounters resulting in antibiotics determined that $50 \%$ were for symptoms $\leq 7$ days ( $95 \%$ CI: $41-58 \%$ ) and $35 \%$ for symptoms $\geq 14$ days (95\% CI: $27-44 \%$ ). Only $29 \%$ (95\% CI: $22-36 \%)$ of encounters resulted in guideline adherent care (30\% PC, 26\% UC, 16\% ED).

Discussion: Among adults with AS in a large, integrated health care system, CT imaging is infrequent but antibiotic treatment is common, even for uncomplicated cases with a short duration of symptoms.

Keywords: acute care improvement, low-value care

PS2-24:

Building Infrastructure to Recruit, Hire and Engage Patient Partners in Research Collaborations

Leah Tuzzio, ${ }^{1}$ Julie E. Richards, ${ }^{1}$ Evette J. Ludman, ${ }^{1}$ Clarissa $\mathrm{Hsu}^{1}$

${ }^{1}$ Group Health Cooperative 
Background/Aims: Funding agencies like the PatientCentered Outcomes Research Institute (PCORI) are requiring that health-related research engage patients in new and more integrated ways, such as employing patient co-investigators. HMORN researchers have often engaged patients in an advisory capacity and are now expanding ways to collaborate. This work builds on Kaiser Permanente Colorado's Institute for Health Research Member Engagement Toolkit and the HMORN Patient Engagement in Research (PER) Interest Group's environmental scan of tools, practices and gaps. The aims of our project are to: 1) develop a toolkit to recruit, hire and orient patient researchers and their research teams, and 2) develop patient community forums to solicit ideas about patient-centered research projects.

Methods: We will conduct semistructured interviews with PCORI research teams, delivery system partners and members of the PER Interest Group. Feedback from these interviews will be used to adapt existing tools or develop new ones to recruit, hire and orient patient investigators and their teams. To develop patient forums, we will work with Group Health's Governance Services, Communication and Clinical Improvement and Prevention departments to utilize existing communication methods and/or create new ones. We will pilot test and evaluate the usability of the resources with project teams submitting new grant proposals.

Results: We will present preliminary insights from these interviews and initial work on forum development. Our presentation will be aimed at both generating additional discussion and feedback and helping apprise the field of practices that are cutting edge in this area of health services research. Already, Group Health teams working on PCORI projects have learned valuable lessons about the benefits of partnering with patient investigators, "They ask great questions and they have blown our assumptions out of the water." We also have learned about important barriers, "Computer skills or knowledge of human subjects research should not be assumed." Our project builds on this experience to improve our capacity to collaborate with patient research partners.

Discussion: The HMORN is well-positioned to be a leader in patient-centered outcomes research. This project builds on the experience, existing resources and current partnerships within the HMORN to develop innovative and proactive patient engagement infrastructure for health research and evaluation.

Keywords: patient engagement, toolkit

\section{PS2-26:}

\section{Delivery System Partnerships Empower Large Pragmatic Trials: The Case of Diabetes Prevention in Kaiser Permanente}

William M. Vollmer, ${ }^{1}$ Carmit K. McMullen, ${ }^{1}$ Ashli OwenSmith, ${ }^{2}$ Steve Fortmann, ${ }^{1}$ Harry Glauber, ${ }^{1}$ David H. Smith, ${ }^{1}$ Cynthia Rand, ${ }^{3}$ Victor Stevens, ${ }^{1}$ Beth Waitzfelder, ${ }^{4}$ Jocelyn Audelo, ${ }^{5}$ Corliss McKeever, ${ }^{6}$ Nangel M. Lindberg ${ }^{1}$
${ }^{1}$ Kaiser Permanente Northwest; ${ }^{2}$ Kaiser Permanente Southeast; ${ }^{3} J o h n s$ Hopkins University; ${ }^{4}$ Kaiser Permanente Hawaii; ${ }^{5}$ Kaiser Permanente Care Management Institute; ${ }^{6}$ African American Health Coalition Inc.

Background/Aims: Successful pragmatic trials require close partnership between researchers and health care organizations. We describe the process by which we engaged clinical and operational leaders from three Kaiser Permanente (KP) regions to design a trial to test alternative population-based models for diabetes prevention.

Methods: The study leveraged ongoing efforts in each region to develop a populationwide approach to diabetes prevention. Working with clinical leaders from KP Northwest (KPNW), we designed an initial study that we then took to the leadership of KP Southeast and KP Hawaii. We also obtained the endorsement of KP's national Care Management Institute (CMI). After submitting a successful letter-of-intent to the Patient-Centered Outcomes Research Institute, we worked with our health plan partners to develop a full proposal that is presently under review.

Results: Throughout the proposal development process, we consulted key regional and national health plan stakeholders on various design issues. We also included the KPNW lead for diabetes management as a full co-investigator and regular member of our weekly planning meetings. As a direct result of these interactions, we dropped our initial three-arm design in favor of a two-arm design because it became clear that our planned low-intensity arm would likely be obsolete by the time the grant was funded. We also decided to focus our study on those at highest risk for progressing to diabetes since this was where the organization clearly intended to focus its greatest energy. Finally we leveraged an ongoing demonstration project being coordinated by the CMI to evaluate an online diabetes prevention curriculum that was seen as a scalable model for providing a proven lifestyle change program to our members nationwide. We incorporated this program into our intervention design and conducted interviews with patients to better understand their perspective regarding the overall usefulness of the program and barriers to participation. To meet the needs of members who preferred face-face classes, we also partnered with local diabetes programs in our communities to provide this option.

Discussion: Our process of stakeholder engagement directly informed the design of the trial and helped to ensure strong institutional support for the study should it be funded.

Keywords: pragmatic trials, stakeholder engagement

\section{STATISTICAL AND RESEARCH METHODS}

\section{D2-3:}

Increasing Efficiency in Creating High-Level Site Comparisons and Populating Cohort Flow Diagrams: The "Cohort Diagram" Macro

Heather M. Tavel, ${ }^{1}$ Nikki M. Carroll, ${ }^{1}$ Heather S. Feigelson, ${ }^{1}$ Marsha A. Raebel ${ }^{1}$ 


\section{${ }^{1}$ Kaiser Permanente Colorado}

Background/Aims: Understanding how a population is selected for an analysis is necessary for understanding potential sources of bias. Searching logs to determine counts for key steps of the process is cumbersome, and re-ordering a flow diagram after all data have been collected is problematic. We developed two tools at Kaiser Permanente Colorado (KPCO) for capturing counts during cohort creation, one of which was refined through a Center for Effectiveness and Safety Research study. These tools are used for comparing similarities and differences in data flowing from multiple health plan sites, for quality assurance, and for populating cohort diagrams for presentations and manuscripts.

Methods: A macro was created with parameters to define a source data set, an output table to hold counts and a step description. An alternate and complementary method of collecting counts was also developed that consists of building a table at the individual level with Boolean flags for each criterion used in cohort selection, which is then summarized into an n-way de-identified frequency table.

Results: The count macro method allows for users to collect any count at any time during the process. Users also specify whether to count rows of a table or distinct values of a specific variable. Multiple output data sets may be used to capture different data flows, such as one dataset for a cohort diagram and another to check for other key quality assurance counts. Tables returned from sites can readily be combined and compared to assess heterogeneity across sites. The n-way frequency table method, while CPU-intensive for large cohort processes, allows for maximum flexibility in the ordering of a cohort diagram. It also adds an extra layer of quality assurance, particularly when paired with a cohort diagram table built using the count macro.

Discussion: These tools have been shared within the KPCO analytic team to aid in data collection and cohort diagram development. They are simple to implement, applicable in distributed data environments and reusable across projects. Reviewing the tables prior to requesting further data can prevent data reruns. Other sites and projects will benefit from increased efficiency by using these tools.

Keywords: quality assurance, programming efficiencies

\section{PD2-10:}

\section{Characterizing Longitudinal Patterns of Breast Cancer Care}

\section{Caroline A. Thompson, ${ }^{1}$ Harold S. Luft ${ }^{1}$ \\ ${ }^{1}$ Palo Alto Medical Foundation Research Institute}

Background/Aims: Cancers with high rates of survival, e.g. breast cancer, are often characterized by treatment periods, or episodes of care, that can continue intermittently for months or years. The effective beginning of a cancer care episode may precede the date a patient obtains a definitive diagnosis. The peridiagnosis phase is often a time of intense resource use that may involve patients seeking care or physician opinions at multiple health care organizations. After the initial episode of treatment, which may involve multiple interventions and cycles, routine posttreatment surveillance may continue indefinitely. Considering the extended length of breast cancer treatment and follow-up, the electronic health record (EHR) for one or more organizations may reflect only part of the long-term care of these patients. In this study we characterize longitudinal patterns of breast cancer care across two health care organizations using a linked EHR database.

Methods: We studied a cohort of 13,377 women with evidence of some breast cancer care, 2000-2013. We distinguished breast cancer-related treatment from other types of care, e.g. diagnosis, second opinions, surveillance. We developed an algorithm to define the customary cancer care periods (routine screening, peridiagnosis, treatment, posttreatment surveillance). We determined number of care episodes for each patient and classified each patient as receiving care at either or both of the health care organizations.

Results: Care period classification identified a subsample of 7,365 patients who received treatment for breast cancer, the rest of the cohort were seen only for diagnosis or posttreatment surveillance. Among those treated, $17 \%$ had more than one treatment episode and $16 \%$ of patients sought care at both organizations. We present details of our classification algorithms and other key findings.

Discussion: By examining specific components of the cancer care episode, we can assess whether breast-related services were provided concurrently or sequentially, and before, during or after the initial diagnosis period. Categorizing cancer-related services by purpose and time offers valuable insights into how care is organized and delivered.

Keywords: electronic health records, care patterns

\section{PS2-29: \\ Increasing the Efficiency of Presenting Study Population Descriptive Characteristics: The "Table 1" Macro}

Nikki M. Carroll, ${ }^{1}$ Heather M. Tavel, ${ }^{1}$ Christina L. Clarke, ${ }^{1}$ J. David Powers, ${ }^{1}$ Heather S. Feigelson, ${ }^{1}$ Marsha A. Raebel $^{1}$

\section{${ }^{1}$ Kaiser Permanente Colorado}

Background/Aims: Basic characteristics of a data set or cohort are often described using a "Table 1." Numerous iterations of this table can be required before the final product is satisfactory. Reproducing this table multiple times can become onerous and increases the chance for data entry errors. Some programmers create their Table 1 through the output delivery system (ODS) in SAS and some output data into Excel. A recently funded Center for Effectiveness and Safety Research project supported us in developing macros to create a "Table 1" macro that can easily accommodate format and content changes and output data in either ODS or Excel format.

Methods: The Kaiser Permanente Colorado analytic team developed two different macros for creating "Table 1," one for frequencies, means and p-value output through ODS into a pdf or rtf file and one for frequencies and means output 
into an Excel file. We describe the features, advantages and disadvantages of each macro.

Results: By converting the Table 1 development process into a macro, we have increased the efficiency of producing and reviewing the results of descriptive analysis while at the same time facilitating different examinations of the data. Both macros have the flexibility to present typical descriptive statistics, such as frequencies, means and comparative p-values. Output from ODS produces a table that is ready to distribute without additional manipulation by the programmer. However, it can be challenging to set up the macro variables correctly. An advantage for the Excel output macro is ease of use, whereas some disadvantages include the time required to format the spreadsheet after data are exported into Excel and moving data into the correct fields. These macros are durable in that they can be applied across multiple projects and variables can be customized for any Table 1 analysis.

Discussion: These macros increase the efficiency of producing and reviewing descriptive analyses and reduce errors that can be introduced from data entry and creating tables de novo. Future work includes combining the macros so that the user can choose the preferred output destination.

Keywords: descriptive statistics, macros

PS2-36:

\section{A Bayesian Approach to Modeling Risk of Hospital Admissions Associated With Schizophrenia Accounting for Underdiagnosis of the Disorder}

Eileen M. Stock, ${ }^{1}$ James D. Stamey, ${ }^{2}$ John E. Zeber, ${ }^{1}$ Alexander W. Thompson, ${ }^{1}$ Laurel A. Copeland ${ }^{1}$

\section{${ }^{1}$ Scott \& White Health System; ${ }^{2}$ Baylor University}

Background/Aims: Schizophrenia is a debilitating serious mental illness (SMI) characterized by a complex array of symptoms. Patients tend to seek treatment only intermittently, contributing to difficulty in diagnosing the disorder. A misdiagnosis may potentially bias and reduce the validity of a study based on recorded diagnoses. It also may impact patient outcomes by delaying receipt of appropriate care. Thus, we present a statistical model to compare the odds of 1-year hospitalization among patients with schizophrenia versus patients with or without other SMIs when schizophrenia is underreported in administrative databases.

Methods: A retrospective study design examined patients seeking care during 2010 in one of 19 care-and-coverage health systems across the United States comprising the Health Maintenance Organization Research Network (HMORN). Bayesian analysis was applied to address the problem of underdiagnosed schizophrenia using a statistical measurement error model that explicitly evaluated the impact of varying assumptions about the extent of underreporting. Results were then compared to a classical multivariable logistic regression model.

Results: Among 87,806 patients, 7.3\% had a SMI, including 114 (1.3 per 1,000) diagnosed with schizophrenia. Admission was greatest among patients with schizophrenia (14\%), followed by other SMIs (12-13\%) and non-SMI patients
$(8 \%, \mathrm{P}<0.01)$. Assuming no underreporting, there was an $87 \%$ greater relative odds of admission associated with schizophrenia (odds ratio: 1.87; 95\% confidence interval: 1.08-3.23). In the Bayesian approach, assuming varying sensitivities, effect sizes were 2-3\% lower with credible interval smaller (reduced by $2-4 \%$ ) than that observed with the classical approach.

Discussion: A delayed diagnosis of schizophrenia can lead to inappropriate treatment and symptom exacerbation, increasing the risk of hospitalization. In the Bayesian approach, reduced association between hospitalization and schizophrenia, as well as other SMIs, was uniformly observed across varying rates of underdiagnosing, meaning prompt diagnosis should lead to lower rates of hospitalization. Although effect sizes may vary across health care systems, the analytical approach has useful applications in other contexts where the identification of patients with a given condition may be subject to underreporting in administrative records.

Keywords: underdiagnosis, Bayesian analysis

PS2-37:

The State of the Census

Christopher D. Mack ${ }^{1}$

${ }^{1}$ Group Health Cooperative

Background/Aims: HMORN institutions are geocoding their patients in a variety of ways with a variety of tools. Neighborhood data from the Census Bureau and American Community Survey can then be used as proxies for data that is not captured, such as socioeconomic status. We are interested in understanding how variation across institutions can affect estimates and variances.

Methods: Descriptive statistics. Comparison of simple linear models with mixed models (i.e. hierarchical linear models or latent variable models).

Results: Results are forthcoming.

Discussion: Conclusions to follow.

Keywords: census, geography

PS2-40:

Using HMORN's Virtual Data Warehouse From Two Health Systems to Identify Risk Factors for Abdominal Aortic Aneurysm

Diane T. Smelser, ${ }^{1}$ Annemarie G. Hirsch, ${ }^{1}$ Meredith W. Lewis, ${ }^{1}$ Jove Graham, ${ }^{1}$ Paul J. Hitz, ${ }^{2}$ Catherine A. McCarty, ${ }^{2}$ Jonathan A. Bock, ${ }^{1}$ Kenneth M. Borthwick, ${ }^{1}$ Gerardus Tromp, ${ }^{1}$ Jacob Mowrey, ${ }^{1}$ Evan Ryer, ${ }^{1}$ James Elmore $^{1}$

\section{${ }^{1}$ Geisinger Health System; ${ }^{2}$ Essentia Health}

Background/Aims: Abdominal aortic aneurysm (AAA) is a leading cause of death in the United States, often undetected until rupture. At least $33 \%$ of ruptured AAA hospitalizations are among women, and $22 \%$ of AAA-related deaths occur in nonsmokers, individuals not covered by current screening 
guidelines. Identifying additional risk factors for AAA would allow for targeted screening of a larger at-risk population. We conducted a retrospective study of electronic health record and claims data from the HMO Research Network's (HMORN) virtual data warehouse (VDW) in two sites: Geisinger Health System (GHS) and Essentia Institute of Rural Health (EIRH). Methods: We used an algorithm that includes CPT and ICD9 codes to classify individuals in the VDW as cases, controls or excludes. This algorithm had a positive predictive value of $94 \%$ and sensitivity of $100 \%$. We extracted demographic, behavioral and clinical covariates, including comorbidities such as peripheral artery disease, diabetes, neoplasms, and pulmonary, kidney and cerebrovascular diseases. Individuals were excluded based on age, no visit in 5 years, genetic conditions or unspecified aneurysm site.

Results: We identified 2,133 AAA cases and 130,289 controls from GHS and 1,986 cases and 196,534 controls from EIRH. Risk factors were similar in direction and magnitude of effect and level of significance across sites, including the novel association of benign neoplasms with AAA.

Discussion: We leveraged the VDW to efficiently demonstrate the transportability of an algorithm for identifying AAA patients, expanding the sample size for studying AAA risk, and replicating our risk factor findings in a second institution. This work was funded in part by National Human Genome Research Institute as both sites are members of the Electronic Medical Records and Genomics (eMERGE) Network (U01HG006382 to Geisinger Clinic and U01HG006389 to Essentia Institute of Rural Health).

Keywords: virtual data warehouse, risk factors

\section{VACCINE EFFECTIVENESS AND SAFETYIINFECTIOUS DISEASES}

\section{B1-4: \\ Interventions to Reduce Hospital-Associated Infections: Comparative Efforts at One HMORN Site and a Local Veterans Affairs Facility}

John E. Zeber, ${ }^{1}$ Laurel A. Copeland, ${ }^{1}$ Chetan Jinadatha, ${ }^{2}$ Eileen M. Stock, ${ }^{1}$ Karen Brust, ${ }^{1}$ John K. Midturi ${ }^{1}$

\section{${ }^{1}$ Scott \& White Health System; ${ }^{2}$ Central Texas Veterans Administration}

Background/Aims: Health care-associated infections (HAI) affect 1.7 million patients and cause 100,000 deaths annually, yet preventive strategies are only partially effective. HAI are often transmitted between health care workers and patients; the sharp increase of antibiotic-resistant organisms compounds the problem. Fortunately, numerous efforts to reduce HAI incidence are being adopted, targeting clinical and environmental cleaning polices. We document current infectious disease work by investigators uniquely positioned within Scott \& White and nearby Central Texas VA. Our involvement in several disinfection strategies and research protocols utilizes evidence-based findings to transform patient care while reducing unnecessary treatment costs.

Methods: Besides aggressive hand-washing and antibiotic stewardship programs at both institutions, ongoing VA initiatives focus on novel pulsed-xenon ultraviolet ray (UV) technologies to eliminate patient room pathogens. Supported by laboratory experiments, this ongoing project examines the clinical efficacy and cost-effectiveness of portable UV devices, gauging implementation challenges. Other decontamination or preventive efforts include air disinfection in operating suites to target surgical site infections (Scott \& White), and copper-infused room surfaces to thwart microbial build-up in high-touch areas (VA). A new veterinary medicine partnership is exploring MRSA transmission via animal contact, a major facet of rural Central Texas life.

Results: Research and quality improvement findings highlight potential barriers in bridging technological advances and health care delivery realties, yet are quite promising. We found a $99.4 \%$ reduction in MRSA microbial counts with evidence of HAI declines where UV devices are routinely deployed; other tests suggest these machines work even absent manual cleaning and without bacteria developing resistance. Carbapenemase-resistant infections dropped $75 \%$ with higher overall treatment response since Scott \& White initiated better HAI screening, selective antibiotic combination therapies (e.g. minocycline plus amikacin) and other infection control precautions.

Discussion: Pragmatic clinical efforts paired with insightful research simultaneously tackle universal health care system concerns, as HAI perniciously evolve with containment strategies. However, mounting evidence provides optimism, encouraging future collaborative endeavors. Our work demonstrates a crucial need to involve multiple stakeholders (clinical leadership, housekeeping, researchers) spurring practical hygiene programs, educational training and creative advances to combat HAI. Future efforts will spotlight implementation and translational progress as we continue pursuing effective models across multiple health systems.

Keywords: hospital-associated infections, novel technology, implementation

\section{D2-5:}

Rapid, Active Surveillance for Medically Attended Acute Gastroenteritis and Norovirus Infection in a Managed Care Environment

Mark A. Schmidt, ${ }^{1}$ Allison L. Naleway, ${ }^{1}$ Elizabeth J. Esterberg, ${ }^{1}$ Emilio E. DeBess,${ }^{2}$ Christianne Biggs,${ }^{2}$ Aron J. Hall ${ }^{3}$

${ }^{1}$ Kaiser Permanente Northwest; ${ }^{2}$ Oregon Health Authority; ${ }^{3}$ Centers for Disease Control and Prevention

Background/Aims: Norovirus is the leading cause of acute gastroenteritis in the United States and is the leading cause of medically attended acute gastroenteritis (MAAGE) among children younger than five years of age. Our primary goal is to describe the community incidence of MAAGE and the proportion of MAAGE encounters due to norovirus within the membership population of Kaiser Permanente Northwest (KPNW). Our secondary goal is to describe transmission of norovirus within households. 
Methods: We are conducting a 12-month active surveillance project in which we identify all KPNW members with MAAGE-associated health care encounters through daily electronic abstraction of the electronic health record system. We are recruiting a sample of this population daily, along with their symptomatic household members, to complete a recruitment survey and collect a stool sample for norovirus testing.

Results: During the four months of our surveillance period, we identified 6,156 members presenting for MAAGE (annualized incidence of 413/100,000). We attempted to reach 1,782 (30\%) potential participants, $525(29 \%)$ of whom were ineligible. Of those remaining, 708 (56\%) refused and 549 (44\%) agreed to participate. Of those agreeing to participate, $453(83 \%)$ provided a sample from which we obtained viral testing results. Among samples tested, we identified 52 (11\%) positive for norovirus. Norovirus positivity was highest among those $0-4$ years of age $(20 \%)$ and lowest among those 65 years or older $(6 \%)$. Thus far, we have recruited 48 household members, $8(15 \%)$ of whom tested positive for norovirus; 7 (88\%) had the same laboratory results as their MAAGE-associated household member.
Discussion: Our observed incidence of MAAGE and proportion of MAAGE encounters due to norovirus infection in the KPNW member population over the first four months of our surveillance period are consistent with results obtained from other studies using varying methodologies and reinforce the high burden of MAAGE and norovirus infection within the community. Our results further support the high transmission of norovirus infection within households. Importantly, our project — with $44 \%$ agreeing to participate and $83 \%$ collecting and returning a stool sample — demonstrates a model for conducting other community-based studies of acute infectious diseases within a managed care system.

Keywords: public health surveillance, norovirus 